Aus der Klinik für Kinder- und Jugendmedizin

(Prof. Dr. med. J. Gärtner)

der Medizinischen Fakultät der Universität Göttingen

\title{
In-vivo-Flussdynamik \\ des Hirnwassers im Spinalkanal - eine Phasenkontrast-Echtzeit-MRT-Studie
}

\author{
INAUGURAL-DISSERTATION \\ zur Erlangung des Doktorgrades \\ der Medizinischen Fakultät der \\ Georg-August-Universität zu Göttingen
}

vorgelegt von

Mareen Konopka

aus

Berlin

Göttingen 2019 
Dekan: $\quad$ Prof. Dr. rer. nat. H.K. Kroemer

\section{Betreuungsausschuss}

Betreuerin: PD Dr. med. S. Dreha-Kulaczewski

Ko-Betreuer: Prof. Dr. rer. nat. J. Frahm

\section{Prüfungskommission}

Referentin: PD Dr. med. S. Dreha-Kulaczewski

Ko-Referent: Prof. Dr. rer. nat. M. Uecker

Datum der mündlichen Prüfung: 10.10.2019 
Hiermit erkläre ich, die Dissertation mit dem Titel „In-vivo-Flussdynamik des Hirnwassers im Spinalkanal - eine Phasenkontrast-Echtzeit-MRT-Studie" eigenständig angefertigt und keine anderen als die von mir angegebenen Quellen und Hilfsmittel verwendet zu haben.

Göttingen, den

Mareen Konopka 
Teile dieser Arbeit wurden bereits veröffentlicht.

Dreha-Kulaczewski S, Konopka M, Joseph AA, Kollmeier J, Merboldt K-D, Ludwig H-C, Gärtner J, Frahm J (2018): Respiration and the watershed of spinal CSF flow in humans. Sci Rep 8, 5594 


\section{Inhaltsverzeichnis}

Abbildungsverzeichnis III

Tabellenverzeichnis $\quad$ V

Abkürzungsverzeichnis $\quad$ VI

1 Einleitung 1

1.1 Funktion des Liquors . . . . . . . . . . . . . . . . . . . . . . . . 1

1.2 Liquorbildung und -resorption . . . . . . . . . . . . . . . . . . 2

1.3 Klinische Bedeutung der Flussdynamik des Hirnwassers . . . . . . . . . . 3

1.4 Bisherige Arbeiten zur Flussdynamik des Hirnwassers . . . . . . . . . . . 4

1.5 Ziel und Fragestellung . . . . . . . . . . . . . . . . . . . . . . . . 5

2 Material und Methoden 6

2.1 Probanden . . . . . . . . . . . . . . . . . . . . 6

2.2 Magnetresonanztomograph . . . . . . . . . . . . . . . 6

2.3 Studiendesign der In-vivo-Phasenkontrast-Echtzeit-MRT-Flussmessung . . 7

2.3.1 Messpositionen . . . . . . . . . . . . . . . . . . 7

2.3 .2 Atemprotokoll . . . . . . . . . . . . . . . . . . 8

2.4 Phasenkontrast-Echtzeit-MRT . . . . . . . . . . . . . . 10

2.5 Datenakquisition . . . . . . . . . . . . . . . . . . . 11

2.6 Datenverarbeitung und -auswertung . . . . . . . . . . . . . 13

2.6.1 CaFuR Software . . . . . . . . . . . . . . . . . . 13

2.6.2 Korrelation der Thorax-/Bauchwandbewegung mit dem

Zeitverlauf des Atemprotokolls . . . . . . . . . . . . . . . . . . 15

2.6.3 Berechnung der Flussvolumina des Hirnwassers . . . . . . . . . 16

2.6.4 Signifikanztest . . . . . . . . . . . . . . . . . 17

2.6 .5 Tiefpassfilter . . . . . . . . . . . . . . . . . . 17

2.6.6 Frequenzanalyse des Hirnwasserflusses . . . . . . . . . . . . . . 17 
3.1 Fluss und Volumen des Hirnwassers entlang des Spinalkanals . . . . . . . 19

3.1.1 Individuelle Flusskurven des Hirnwassers . . . . . . . . . . . . . . 20

3.1.2 Geschwindigkeit, Fläche der ROI und Fluss des Hirnwassers im Gruppenmittel . . . . . . . . . . . . . . . . . . . . . 24

3.1.3 Individuelle Volumenverschiebung des Hirnwassers während forcierter Atmung . . . . . . . . . . . . . . . . . . . . . . . . . . 28

3.1.4 Gruppenmittel der Volumenverschiebung des Hirnwassers während forcierter Atmung . . . . . . . . . . . . . . . . . . . . . 29

3.2 Anteil der respiratorischen und kardialen Frequenzkomponenten am Fluss des Hirnwassers während forcierter Atmung . . . . . . . . . . . . . . . . . 32

4 Diskussion $\quad 38$

4.1 Fluss und Volumen des Hirnwassers entlang des Spinalkanals . . . . . . . 38

4.1 .1 Einordnung der Ergebnisse . . . . . . . . . . . . . . . . . . 38

4.1.2 Interpretation der Ergebnisse . . . . . . . . . . . . . . . . . 39

4.2 Kardialer versus respiratorischer Flussantrieb unterschiedliche Dominanz in Abhängigkeit von der Lokalisation . . . . . . 41

4.3 Limitationen und Fehlerquellen . . . . . . . . . . . . . . . . . . . . 43

4.3.1 Fläche der ROI . . . . . . . . . . . . . . . . . . . . . 43

4.3 .2 Datenverwertung . . . . . . . . . . . . . . . . 43

4.3 .3 Messebene . . . . . . . . . . . . . . . . . . 43

4.3.4 Zeitliche Begrenzung der forcierten Atmung . . . . . . . . . . . . . 44

4.3 .5 Atemprotokoll . . . . . . . . . . . . . . . . . . . . . . . 44

5 Zusammenfassung $\quad 45$

$\begin{array}{lll}6 & \text { Anhang } & 47\end{array}$

7 Literaturverzeichnis $\quad 55$ 


\section{Abbildungsverzeichnis}

13 Tesla MRT . . . . . . . . . . . . . . . . 7

2 Messpositionen der MRT-Flussmessungen $\ldots \ldots \ldots \ldots$

3 Atemprotokoll . . . . . . . . . . . . . . . . . . . 9

4 Magnitudenbild und korrespondierendes Phasenbild einer Phasenkontrastflussmessung in Position L2 während forcierter Exspiration . . . . . . . . . 11

5 Magnitudenbild und korrespondierendes Phasenbild einer Phasenkontrastflussmessung sowie die durchschnittliche Geschwindigkeit innerhalb der ROI, Fläche der ROI und Fluss des Hirnwassers in Position L4 während forcierter Atmung . . . . . . . . . . . . . . . . . . . 14

6 Magnitudenbild mit definierter $\mathrm{ROI}$ sowie Änderung der Signalintensität in der ROI über der Bauchwand in Position L4 während forcierter Atmung . . 16

7 Flusskurve des Hirnwassers sowie korrespondierende Phasendifferenzbilder in Position L4 während forcierter Atmung . . . . . . . . . . . 20

8 Flusskurven des Hirnwassers in allen Messpositionen des Probanden \#17 22

9 Geschwindigkeit des Hirnwassers gemittelt über 19 Probanden entlang des Spinalkanals . . . . . . . . . . . . . . . . . . . . . . 25

10 Fläche der ROI gemittelt über 19 Probanden entlang des Spinalkanals . . 26

11 Fluss des Hirnwassers gemittelt über 19 Probanden entlang des Spinal-

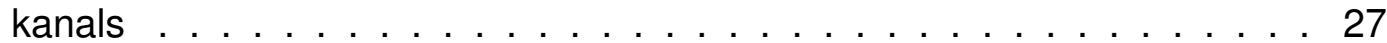

12 In- und exspiratorische Volumina sowie Netto-Volumina des Hirnwassers während forcierter Atmung entlang des Spinalkanals der Probanden \#17 und $\# 1$. . . . . . . . . . . . . . . . . . . . . . . . . . . . . . 29

13 In- und exspiratorische Volumina sowie Netto-Volumina des Hirnwassers während forcierter Atmung entlang des Spinalkanals gemittelt über 19 Probanden . . . . . . . . . . . . . . . . . . . . . . . 31

14 Frequenzspektrum des Flusssignals des Hirnwassers in den Positionen C5 und Th12 des Probanden \#17

15 Frequenzspektrum des Flusssignals des Hirnwassers in allen Messpositionen des Probanden $\# 17 \ldots \ldots$. . . . . . . . . . . . . . 34

16 Frequenzspektrum des Flusssignals des Hirnwassers in allen Messpositionen gemittelt über 19 Probanden 
17 Anteil der respiratorischen und kardialen Frequenzen relativ zur Summe aller Frequenzen in allen Messpositionen gemittelt über 19 Probanden . . 36

A1 Flusskurven des Hirnwassers in allen Messpositionen mit Tiefpassfilter des Probanden $\# 17$. . . . . . . . . . . . . . . . . . . . . . 47

A2 Flusskurven des Hirnwassers in allen Messpositionen des Probanden \#1 . 48

A3 Flusskurven des Hirnwassers in allen Messpositionen des Probanden \#19 49 


\section{Tabellenverzeichnis}

1 Sequenzparameter der MRT-Flussmessungen $\ldots \ldots \ldots$

A1 Geschwindigkeitskodierung/VENC $\left(\mathrm{cm} \mathrm{s}^{-1}\right)$ aller Flussmessungen des Hirnwassers . . . . . . . . . . . . . . . . 50

A2 Volumina des Hirnwassers (ml) jedes Probanden (\#1-19) entlang des Spinalkanals (C3-L4) während forcierter Atmung . . . . . . . . . . . . . . . 51

A3 Signifikante Unterschiede ( $p$-Wert $\leq 0,05)$ zwischen in- und exspiratorischen Volumina sowie Netto-Volumina (ml) des Hirnwassers im Gruppenmittel aller Messpositionen während forcierter Atmung . . . . . . . . . . 52

A4 Relativer Spektralanteil (\%) bei 0,2 Hz während forcierter Atmung . . . . . 53

A5 Relativer Spektralanteil (\%) bei 0,8 bis 1,8 Hz während forcierter Atmung . 54 


\section{Abkürzungsverzeichnis}

\begin{tabular}{ll} 
C1-C3 & Halswirbelkörper 1-3 \\
C5-C7 & Halswirbelkörper 5-7 \\
EX & Exspiration \\
FLASH & fast low angle shot \\
FOV & field of view \\
HASTE & half-Fourier acquisition single-shot turbo spin-echo \\
IN & Inspiration \\
L2 & Lendenwirbelkörper 2 \\
L4 & Lendenwirbelkörper 4 \\
MRT & Magnetresonanztomographie \\
n. a. & nicht auswertbar \\
NLINV & iterativ regularisierte nichtlineare Inversion \\
ROI & region of interest \\
SD & Standardabweichung \\
TE & echo time \\
Th1-Th2 & Brustwirbelkörper 1-2 \\
Th4-Th6 & Brustwirbelkörper 4-6 \\
Th8-Th12 & Brustwirbelkörper 8-12 \\
TR & repetition time \\
VENC & velocity encoding \\
w. E. & willkürliche Einheiten \\
ZYK & Atemzyklus \\
\hline
\end{tabular}




\section{Einleitung}

Liquor cerebrospinalis (von hier an vereinfachend Liquor oder Hirnwasser genannt) ist eine farblose, wasserklare, eiweiß- und zellarme Flüssigkeit, die im gesamten zentralen Nervensystem vorkommt (Trepel 2015). Sie schützt Gehirn und Rückenmark vor mechanischen Einwirkungen und dient u. a. dem Stoffwechsel zentraler Nervenzellen (Bechmann et al. 2012; Trepel 2015).

In dieser Arbeit wird die Flussdynamik des Hirnwassers entlang des gesamten Spinalkanals während der forcierten Atmung quantitativ untersucht.

\subsection{Funktion des Liquors}

Das Hirnwasser schützt das Gehirn gegen die Kräfte des eigenen Gewichts (Trepel 2015).

Des Weiteren hat der Liquor als Teil des erst kürzlich beschriebenen glymphatischen Systems (Nedergaard 2013) eine wichtige Funktion bei der Konstanthaltung des extrazellulären Milieus (Trepel 2015) der Hirnzellen wie der Aufrechterhaltung des SäureBase-Haushalts (Silverberg et al. 2003) und ist an der Entwicklung des zentralen Nervensystems beteiligt (Miyan et al. 2003). Der Übertritt des Liquors aus den paravaskuIären Räumen in die Hirnvenen dient u. a. dem Abtransport schädlicher Metabolite aus Neuronenaktivitäten, der Weiterleitung hormoneller und immunmodulatorischer Signale als Teil des neuroendokrinen Systems sowie der Aufrechterhaltung des hydrostatischen Drucks (Silverberg et al. 2003; Johanson et al. 2008; Jessen et al. 2015; Spector et al. 2015; Trepel 2015; Tumani et al. 2017).

Eine Störung dieser Clearancefunktion des Liquors spielt nach neueren Auffassungen in der Ätiologie und Pathogenese neurodegenerativer Erkrankungen wie der AlzheimerDemenz eine entscheidende Rolle (Silverberg et al. 2003; Silverberg et al. 2006; Jessen et al. 2015; Illes 2017). 


\subsection{Liquorbildung und -resorption}

Der Liquor verteilt sich im Liquorraum, wobei anatomisch äußere von inneren Liquorräumen unterschieden werden. Die äußeren Liquorräume umfassen die Subarachnoidalräume des Gehirns und Rückenmarks. Die inneren setzen sich aus den vier intrazerebralen Hirnkammern, den sog. Ventrikeln, dem Aquaeductus mesencephali (von hier an vereinfachend Aquädukt genannt) und dem Zentralkanal des Rückenmarks zusammen (Bechmann et al. 2012).

Produktion und Kontrolle der Zusammensetzung des Hirnwassers erfolgt in den Plexus choroidei in den vier Ventrikeln, besonders in denen der Seitenventrikel (Johanson et al. 2008; Spector et al. 2015; Trepel 2015). Diese Plexus sind arteriovenöse Gefäßkonvolute, die von einem speziellen Epithel - dem Ependym - bedeckt sind (Trepel 2015). Weitere molekularbiologische Details zur Hirnwasserproduktion finden sich bei Brown et al. 2004 und Damkier et al. 2013.

Die vier Ventrikel sind miteinander verbunden. Die inneren Liquorräume stehen über drei Recessus mit Öffnungen (paarige Aperturae laterales [Foramina Luschkae], unpaarige Apertura mediana [Foramen Magendii]) im IV. Ventrikel mit den äußeren Liquorräumen in Verbindung. Das Hirnwasser kann hier zwischen Ventrikeln und Subarachnoidalräumen zirkulieren (Trepel 2015).

Mit einer Gesamtmenge von ca. $150 \mathrm{ml}$ füllt das Hirnwasser innere und äußere Liquorräume aus. Hierbei befinden sich ca. 20 \% des Hirnwassers in den Ventrikeln und $80 \%$ in den äußeren Liquorräumen (Trepel 2015). Insgesamt werden ca. 500 ml Hirnwasser innerhalb von 24 Stunden gebildet und das Volumen wird etwa dreimal täglich ausgetauscht (Bechmann et al. 2012; Galarza et al. 2014).

Der Liquor wird von den äußeren Liquorräumen über zwei Wege resorbiert: Im Schädelbereich gelangt er sowohl über Ausstülpungen der Hirnhäute (Pacchioni-Granulationen) in die venösen Blutleiter der harten Hirnhaut (Trepel 2015) als auch über Verbindungen im Nasen-Rachen-Raum (Siebplatte) und weiter über die Nasenschleimhaut in das Lymphgefäßsystem der Kopf-/Halsregion. Von dort fließt er in den venösen Blutkreislauf (Koh et al. 2005). Im Wirbelkanal- und Schädelbasisbereich wird der Liquor über die Durataschen der Hirn- und Spinalnerven ins Venen- und Lymphgefäßsystem abgeleitet (Bechmann et al. 2012; Trepel 2015). 


\subsection{Klinische Bedeutung der Flussdynamik des Hirnwassers}

Angesichts der zahlreichen Funktionen des Hirnwassers und der Beteiligung an der Entwicklung des zentralen Nervensystems (Miyan et al. 2003) wirken sich pathologische Änderungen der Dynamik des Hirnwassers kritisch oder sogar lebensbeeinträchtigend auf die Bildung, das Volumen, die Zusammensetzung, die Resorption und den Fluss des Hirnwassers aus (Stadlbauer et al. 2010). Es wird angenommen, dass Störungen des Hirnwasserflusses den Flüssigkeitshaushalt sowie Stoffwechselprozesse des Gehirns ungünstig beeinflussen, was wiederum mit Erkrankungen wie Normaldruckhydrozephalus und Alzheimer-Demenz assoziiert ist (Silverberg et al. 2003; Silverberg et al. 2006).

Darüber hinaus dienen Beobachtungen zur Flussdynamik des Hirnwassers anderen Feldern der Wissenschaft wie beispielsweise Studien über den Transport von Stammzellen während der Hirnentwicklung (Stadlbauer et al. 2010) oder der Entwicklung neuer medikamentöser Therapiekonzepte (Misra et al. 2003; Stearns et al. 2005; Askar et al. 2007).

Die nicht-invasive In-vivo-Phasenkontrast-Echtzeit-Magnetresonanztomographie (MRT) eröffnet durch ihre hohe zeitliche und räumliche Auflösung neue Möglichkeiten für die quantitative Untersuchung der Hirnwasserdynamik ohne Abhängigkeit von einem physiologischen Trigger (in dem Fall Synchronisation mit dem Herzzyklus). Die Übertragung der Phasenkontrast-Echtzeit-MRT in den klinischen Alltag ist von großer Bedeutung, um die pathophysiologischen Mechanismen, die z. B. Erkrankungen des Rückenmarks wie der Syringomyelie zugrunde liegen, aufzudecken. Des Weiteren können möglicherweise Erkrankungsursachen von verschiedenen Formen des kindlichen Hydrozephalus oder der Arnold-Chiari-Malformationen Typ I (Stadlbauer et al. 2010; Battal et al. 2011; DrehaKulaczewski et al. 2018) erkannt werden. Die Eröffnung neuer Therapieoptionen und deren Evaluationen (Dreha-Kulaczewski et al. 2018) sowie Einschätzungen zum Progress von Erkrankungen (Stadlbauer et al. 2010) sind möglich. 


\subsection{Bisherige Arbeiten zur Flussdynamik des Hirnwassers}

Der Ursprung der Bewegung des Liquors wird schon lange kontrovers diskutiert (O'Connell 1943; Bering 1955; Du Boulay 1966; Du Boulay et al. 1972).

Frühe Arbeiten nutzten die Myelographie (Reitan 1941) oder invasive Druckmessungen z. T. mit Hustenmanövern. Letztere zeigten, dass die Atmung die Bewegung des Liquors durch thorakale Druckänderungen beeinflusst (Williams 1976; Williams 1981 a; Williams 1981 b).

In den vergangenen Jahren wurden in zahlreichen Studien mittels Elektrokardiographiesynchronisierter Phasenkontrast-MRT Parameter des Hirnwasserflusses untersucht. Hierbei werden zunächst die Daten aus mehreren Herzzyklen in Abhängigkeit von ihrem jeweiligen zeitlichen Abstand zur R-Zacke im Elektrokardiogramm retrospektiv umsortiert und zu wenigen synthetischen Herzzyklen zusammengefasst. Aus diesen werden anschließend die Geschwindigkeitswerte des Hirnwassers berechnet. Die Arbeiten konzentrierten sich auf herzsynchrone Bewegungen des Hirnwasserflusses meist im Aquädukt. Während der Systole wurde ein Fluss des Hirnwassers nach kaudal und während der Diastole ein Fluss nach kranial beobachtet (Nitz et al. 1992; Schroth und Klose 1992; Greitz et al. 1993). Diese Methode wird von signifikanten Einschränkungen begleitet. Nicht herzsynchrone Bewegungen und physiologische Vorgänge, wie die Atmung, sind dieser Methode nicht zugänglich (Joseph et al. 2014; Dreha-Kulaczewski et al. 2015; Yildiz et al. 2017).

Seit der Einführung flusssensitiver MRT-Sequenzen gibt es nur wenige Arbeiten, die nicht Herzzyklus-synchronisiert den Einfluss der Atmung und der Herzaktion auf die Bewegung des Hirnwassers unter Verwendung echoplanarer Bildgebung (Klose et al. 2000; Friese et al. 2004; Kao et al. 2008) oder mittels Spin-Markierung (Yamada et al. 2013) untersucht haben. Ferner wurde die Geschwindigkeit des Liquorflusses in den genannten Arbeiten nicht quantifiziert und bis auf Arbeiten von Friese et al. 2004 nur an intrakraniellen Messpositionen gemessen. In einer jüngeren Studie wurde mittels Phasenkontrast-Echtzeit-MRT, basierend auf echoplanarer Bildgebung, die Geschwindigkeit und die Richtungsänderung des Liquors im Gehirn und den basalen Zisternen mit vorgegebenem Atemprotokoll evaluiert (Chen et al. 2015). 
Die Arbeitsgruppe um Dreha-Kulaczewski führte quantitative Flussmessungen des Liquors mit hochaufgelöster Phasenkontrast-Echtzeit-MRT während der forcierten Atmung zunächst an intrakraniellen Messpositionen (Dreha-Kulaczewski et al. 2015) und anschließend sowohl intrakraniell (Aquädukt) als auch in den subarachnoidalen Räumen des Spinalkanals im zervikalen (C3) und thorakalen (Th2 und Th5) Bereich (DrehaKulaczewski et al. 2017) durch. Sie beobachteten, dass die Atmung und hier vor allem die Inspiration der eigentliche Motor für die Bewegung des Liquors ist (Dreha-Kulaczewski et al. 2015; Dreha-Kulaczewski et al. 2017).

\subsection{Ziel und Fragestellung}

Ziel der vorliegenden Arbeit ist es, die Flussdynamik des Hirnwassers während der forcierten Atmung mittels flusssensitiver Phasenkontrast-Echtzeit-MRT erstmalig entlang des gesamten Spinalkanals zu untersuchen.

Spezielle Fragestellungen hierbei sind:

1. In welche Richtung fließt das Hirnwasser in den einzelnen Positionen entlang des Spinalkanals während der forcierten In- und Exspiration? Wieviel Hirnwasser fließt dabei? (s. Kapitel 3.1)

2. Welchen Anteil haben jeweils Atmung und Herzaktion am Antrieb des Hirnwasserflusses entlang des Spinalkanals während der forcierten In- und Exspiration? (s. Kapitel 3.2) 


\section{Material und Methoden}

Im folgenden Kapitel werden die Arbeitsschritte zur quantitativen Flussmessung des Hirnwassers im Spinalkanal mittels Phasenkontrast-Echtzeit-MRT beschrieben.

\subsection{Probanden}

Vor Beginn der experimentellen Studie lag das Votum der Ethikkommission (Aktenzeichen 18/2/14) der Universitätsmedizin Göttingen vor. Das Studienprotokoll entsprach der Deklaration von Helsinki.

Es wurden insgesamt 14 männliche und 6 weibliche gesunde Probanden im Alter von 23 bis 34 Jahre $(27,2 \pm 3,2$ Jahre [Mittelwert \pm Standardabweichung (SD)]) ohne bekannte Kontraindikationen (Herzschrittmacher, Innenohr-Implantate, ferromagnetische Prothesen etc.) für die Durchführung einer MRT-Untersuchung rekrutiert. Nach ausführlicher und detaillierter Aufklärung willigten die Probanden zur Teilnahme an der Studie schriftlich ein. Zum Studienzeitpunkt lagen anamnestisch keine pulmonalen oder kardialen Einschränkungen vor. Zusätzlich wurden die Probanden auf die Pseudonymisierung ihrer Daten hingewiesen.

\subsection{Magnetresonanztomograph}

Die Messungen wurden in einem 3 Tesla MRT (Magnetom Prisma Fit, Siemens Healthcare, Erlangen, Deutschland, Abb. 1) durchgeführt. Die Lagerung der Probanden erfolgte in Rückenlage auf der MRT-Liege. Für die visuelle Kontrolle der Atemfrequenz wurde der geräteeigene Respirationsgürtel in Höhe des Zwerchfells angelegt. Die Messungen erfolgten von kaudal nach kranial. Gegen den hohen Lärmpegel während der Messung erhielten die Probanden einen Hörschutz. Eine Alarmklingel ermöglichte ihnen, bei auftretenden Problemen während der Messung mit den Studienleitern im Konsolenraum in Kontakt zu treten. Diese waren mit den Probanden per Mikrofon in Verbindung. 


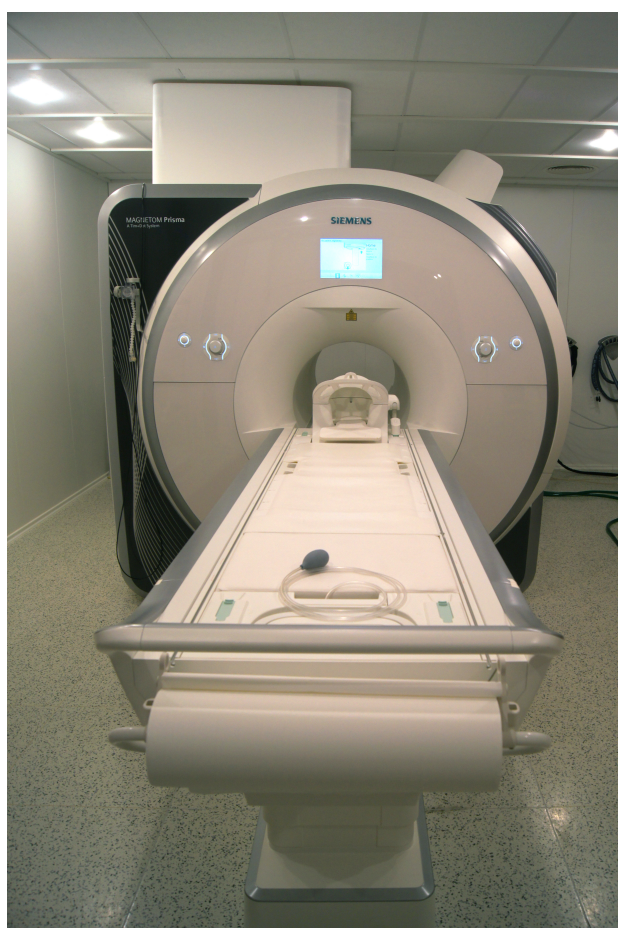

Abbildung 1: 3 Tesla MRT (Magnetom Prisma Fit, Siemens Healthcare, Erlangen, Deutschland) mit ausgefahrener Patientenliege und integrierter 32-Kanal-Rückenspule im Vordergrund und 64Kanal-Kopfspule. Foto mit freundlicher Genehmigung von Irene Böttcher-Gajewski, Max-PlanckInstitut für Biophysikalische Chemie Göttingen, 2016.

\subsection{Studiendesign der In-vivo-Phasenkontrast-Echtzeit- MRT-Flussmessung}

\subsubsection{Messpositionen}

Als Messpositionen für die MRT-Flussmessungen in den subarachnoidalen Räumen wurden im zervikalen (C) Bereich des Spinalkanals die Wirbelkörper C3 und C5, in den thorakalen (Th) Regionen Th1, Th4, Th6, Th8, Th10 und Th12 sowie lumbal (L) L2 und L4 ausgewählt (Abb. 2). 


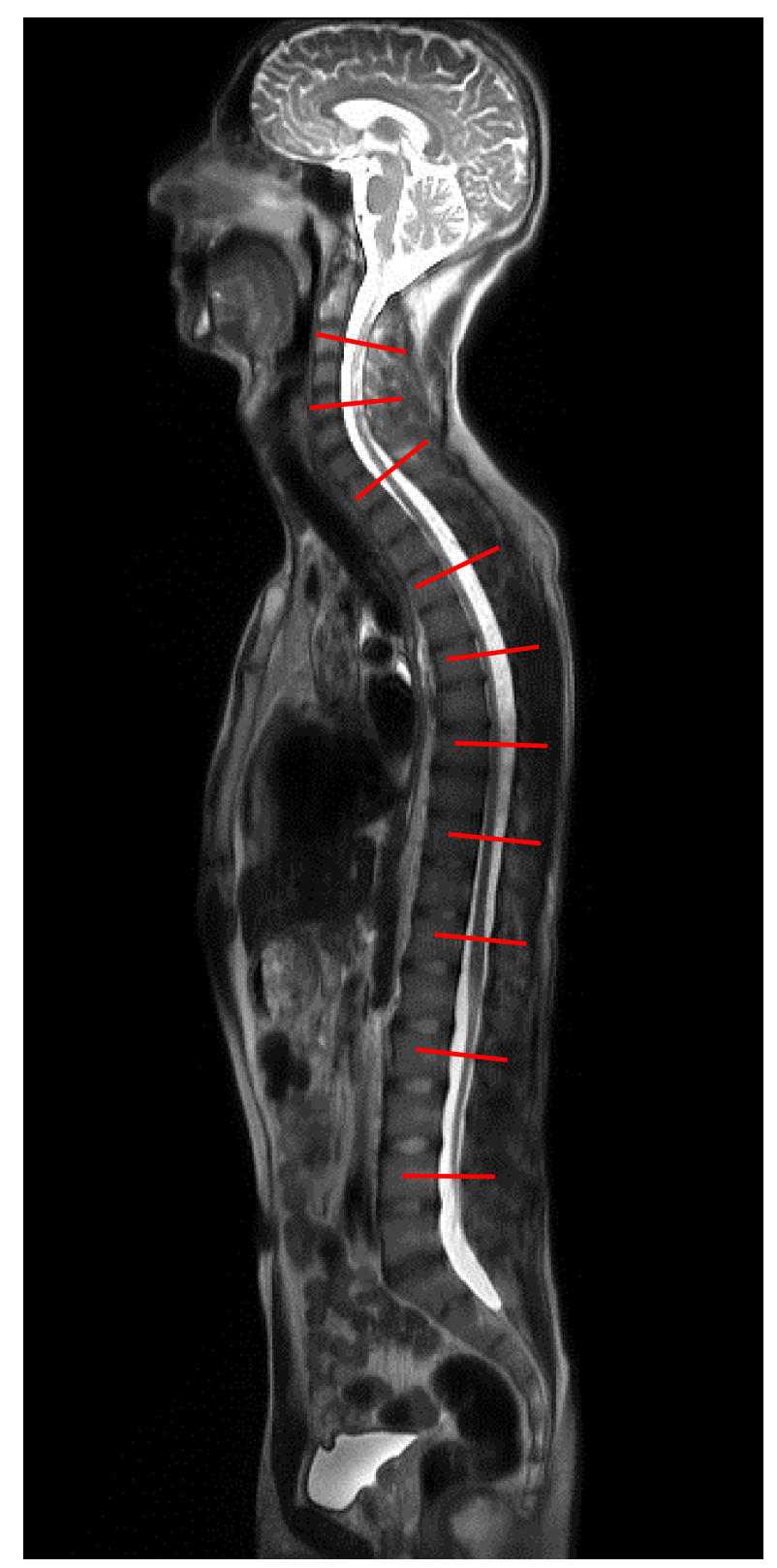

Abbildung 2: Messpositionen C3 bis L4 (rote Markierungen) der MRT-Flussmessungen. T2gewichtete Aufnahme des Spinalkanals $\left(1,4 \times 1,4 \times 6,0 \mathrm{~mm}^{3}\right)$ aufgenommen mit half-Fourier acquisition single-shot turbo spin-echo (HASTE, Siemens).

\subsubsection{Atemprotokoll}

Die Probanden wurden aufgefordert, während der MRT-Flussmessung eine animierte Präsentation des Atemprotokolls (Abb. 3a) zur reproduzierbaren Durchführung der forcierten Atmung zu befolgen. Diese wurde innen mittels eines Projektor auf einer Mattscheibe abgebildet. Die Präsentation (Abb. 3a) wurde zusammen mit der MRTFlussmessung gestartet. Vor der eigentlichen Messung wurde der Ablauf des Atemprotokolls mehrfach geübt. 
Das 40-sekündige Atemprotokoll (Abb. 3b) unterteilte sich in 3 Phasen:

Phase 1: 10 Sekunden normale Atmung

Phase 2: forcierte Atmung, bestehend aus vier Zyklen à 5 Sekunden,

2,5 Sekunden forcierte Inspiration und

2,5 Sekunden forcierte Exspiration, insgesamt 20 Sekunden

Phase 3: 10 Sekunden normale Atmung

a

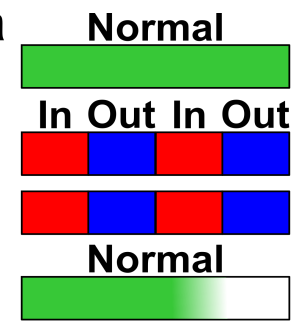

b

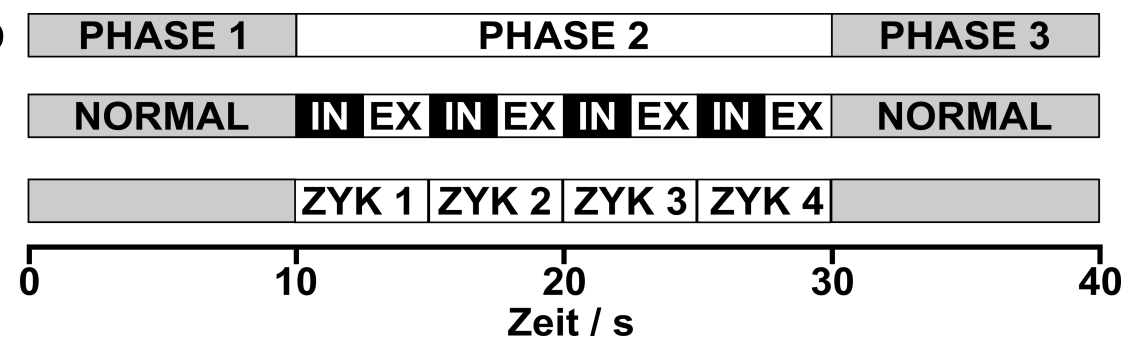

Abbildung 3: (a) Animierte Präsentation des Atemprotokolls zur Anleitung der Probanden während der Messung (normal atmen, einatmen, ausatmen). Die Balken waren bewegungsanimiert und „füllten" sich von links nach rechts. (b) Schematische Darstellung des 40-sekündigen Atemprotokolls: Phase 1: Sekunde 0-10 normale Atmung, Phase 2: Sekunde 10-30 forcierte Atmung, Phase 3: Sekunde 30-40 normale Atmung. Die forcierte Atmung bestand aus vier Atemzyklen (ZYK 1 bis ZYK 4) à 5 Sekunden. Ein forcierter Atemzyklus (ZYK) setzte sich aus je 2,5 Sekunden forcierter Inspiration (IN) und forcierter Exspiration (EX) zusammen.

Die exakte Durchführung des Atemprotokolls wurde während der Messung visuell anhand der Thorax- und Bauchwandbewegung auf den anatomischen Magnitudenbildern und durch Aufzeichnung des Atemsignals mittels Respirationsgürtel überprüft. Zudem wurden die Probanden gefragt, ob sie das Atemprotokoll korrekt eingehalten haben. Gegebenenfalls wurde die Messung wiederholt.

Der zeitliche Rahmen einer MRT-Probandenmessung, einschließlich Aufklärung, lag bei 2,5 bis 3 Stunden. 


\subsection{Phasenkontrast-Echtzeit-MRT}

Das Verfahren der Phasenkontrast-Echtzeit-MRT kombiniert eine schnelle radiale FLASHSequenz (fast low angle shot-Sequenz) mit zusätzlichen flusskodierenden Gradientenmomenten (Joseph et al. 2012; Joseph et al. 2014; Untenberger et al. 2016). Zur Beschleunigung der Datenaufnahme wird der Datenraum (k-Raum) hochgradig unterabgetastet (Uecker et al. 2010) und mittels iterativ regularisierter nichtlinearer Inversion (NLINV) rekonstruiert.

Generell gilt, dass das magnetische Moment von Protonen im konstanten Magnetfeld des Scanners präzidiert. Im Falle von Protonen, die sich mit konstanter Geschwindigkeit (z. B. im Hirnwasser) im Magnetfeld bewegen, erfährt die Präzessionsbewegung durch die Flusskodierung einen zusätzlichen Phasenschub proportional zur Fließgeschwindigkeit. Dies erfolgt bei jedem zweiten Bild, sodass zwei (komplexwertige) Bildserien in alternierender Abfolge aufgenommen werden: eine mit Geschwindigkeitsinformation und eine Referenzserie als Nulllinie. Die Geschwindigkeitskarte ergibt sich aus der Phasendifferenz zwischen den flusskodierten Bildern und den jeweils folgenden Referenzbildern (Bryant et al. 1984). Der Magnitudenanteil der Bilder zeigt hingegen die Anatomie. Aus den zwei Bildserien entsteht eine neue komplexwertige Bildserie, dessen Phasenwerte die Geschwindigkeitskarten ergeben (Abb. 4). Welche Geschwindigkeitsspanne dabei gemessen werden kann, hängt von der Stärke des flusskodierenden Gradienten ab (velocity encoding, VENC). Je stärker der Gradient, desto feiner die Geschwindigkeitsabstufung, desto kleiner die messbare Geschwindigkeitsspanne und länger die Aufnahmezeit pro Bild.

Bei den Messungen im Rahmen dieser Arbeit erfolgte die Geschwindigkeitskodierung ausschließlich entlang der Richtung senkrecht zur Bildebene (through-plane). 


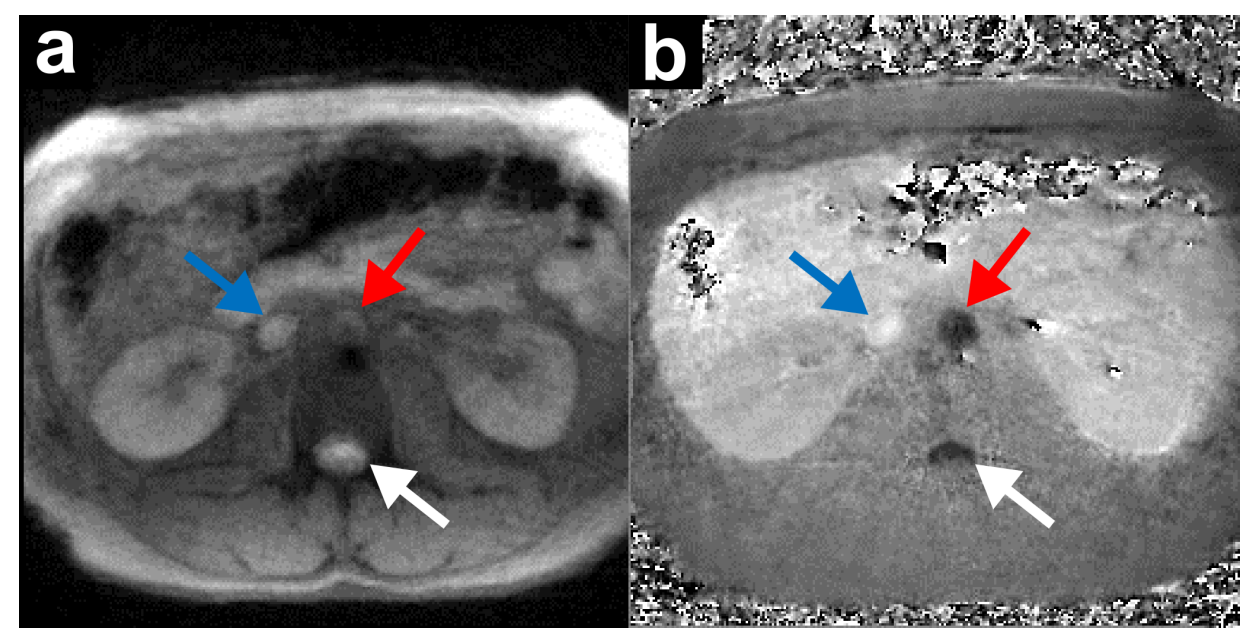

Abbildung 4: (a) Transversales Magnitudenbild und (b) korrespondierendes Phasenbild einer Phasenkontrastflussmessung in Position L2 während forcierter Exspiration. In der Phasenkontrastkarte entsprechen helle Graustufen nach kranial gerichteter Hirnwasserbewegung (Signal hell = Flussrichtung nach kranial) und dunkle entsprechend nach kaudal (Signal dunkel $=$ Flussrichtung nach kaudal). Siehe in der Phasenkontrastkarte den nach kaudal gerichteten Liquorfluss (weißer Pfeil), den nach kaudal gerichteten Fluss in der Aorta abdominalis (roter Pfeil) sowie den nach kranial gerichteten Fluss in der Vena cava inferior (blauer Pfeil).

Für die Rekonstruktion der Phasenkontrastkarten wurde eine parallelisierte Version des NLINV-Algorithmus verwendet (Schaetz und Uecker 2012). Dafür erfolgte die Bildberechnung auf einem bypass-Computer (sysGen/TYAN Octuple-GPU, Sysgen, GeForce GTX TITAN, Nvidia) mit direkter Datenleitung vom Scanner nahezu zeitgleich zur Datenakquisition (Online-Rekonstruktion).

\subsection{Datenakquisition}

Die Datenaufnahme erfolgte im zervikalen Bereich über eine 64-Kanal-Kopfspule und auf Höhe des thorakalen und lumbalen Spinalkanals mittels einer 18-Kanal-Thoraxspule sowie einer 32-Kanal-Rückenspule (8 Segmente mit jeweils 4 Spulenelementen). Je nach Messposition entlang des Spinalkanals wurden geeignete Spulensegmente der Rückenspule zugeschaltet.

Aus den sagittalen und transversalen Lokalisierungsbildern (radial multislice FLASH) wurde die endgültige Messebene so definiert, dass diese senkrecht zum Spinalkanal lag und beidseits keine Spinalkanalnervenaustritte sichtbar waren. 
Die MRT-Flussmessungen der Studie wurden mit folgenden Parametern durchgeführt (Tab. 1):

Tabelle 1: Sequenzparameter der MRT-Flussmessungen

\begin{tabular}{lcc}
\hline Parameter & Position C3 & Position C5-L4 \\
\hline Ortsauflösung & $1,0 \times 1,0 \times 5,0 \mathrm{~mm}^{3}$ \\
Field of view (FOV) & $192 \times 192 \mathrm{~mm}^{2}$ & $256 \times 256 \mathrm{~mm}^{2}$ \\
Echo time (TE) & $4,55 \mathrm{~ms}$ & $4,53 \mathrm{~ms}$ \\
Repetition time (TR) & $5,68 \mathrm{~ms}$ \\
Flip-Winkel & $10^{\circ}$ \\
VENC & $10-40 \mathrm{~cm} \mathrm{~s}^{-1}$ (s. Tab. A1, Anhang) \\
Zeitauflösung & $125 \mathrm{~ms}$ \\
k-Raum-Abtastung & radial (11 Speichen) \\
\hline VENC (velocity encoding) &
\end{tabular}

In Abhängigkeit von der maximal zu erwartenden Geschwindigkeit des Liquorflusses wurden die Flusskodierungsgradienten vorab auf Werte eingestellt, die einer Geschwindigkeitskodierung von 10-40 $\mathrm{cm} \mathrm{s}^{-1}$ entsprachen (Tab. A1, Anhang), wie bereits bei Flussmessungen des Liquors (Aquädukt, C3, Th2, Th5) beschrieben (Dreha-Kulaczewski et al. 2017).

Für jede Messposition erfolgte zunächst eine Messung. Diese wurde bei Auftreten von Rekonstruktionsartefakten oder bei Auftreten von sog. phase wrap-Artefakten (die gemessene Flussgeschwindigkeit übersteigt den Maximalwert des eingestellten Geschwindigkeitsfensters) wiederholt - mit entsprechend angepassten Parametern. Auch wenn ein Proband das Atemprotokoll nicht zeitgerecht befolgte, wurde die Messung erneut durchgeführt. Bildserien, in denen Artefakte durch Anpassung der Messparameter nicht beseitigt werden konnten, ein Rekonstruktionsartefakt weiterhin bestand oder die Zielstruktur nicht sicher erkennbar war, wurden von der Auswertung ausgeschlossen. 


\subsection{Datenverarbeitung und -auswertung}

\subsubsection{CaFuR Software}

Für die quantitative Analyse der Echtzeit-MRT-Flussdaten wurde die CaFuR v.1.1 Software (Fraunhofer MEVIS, Bremen, Deutschland) verwendet, die speziell für die Analyse von Echtzeit-MRT-Daten entwickelt wurde.

Um einen Datensatz zu analysieren, wurde manuell ein initialer Bildbereich (region of interest, ROI) in dem Gebiet mit dem stärksten Flusssignal definiert. Die Einzeichnung der ROI kann dabei sowohl im Magnitudenbild als auch im Phasendifferenzbild erfolgen. Anschließend propagiert das Programm automatisch die manuell eingezeichnete Segmentationslinie unter Berücksichtigung der eventuellen Formänderungen (beispielsweise um ein Gefäß) über alle nachfolgenden Bilder der Messung (Chitiboi et al. 2014).

Nach der automatischen Übertragung erfolgte eine visuelle Überprüfung der ausgewählten Bildbereiche aller Phasen und, falls erforderlich, eine manuelle Korrektur.

Die Software berechnet verschiedene Parameter wie die minimale, maximale und durchschnittliche Geschwindigkeit $\left(\mathrm{cm} \mathrm{s}^{-1}\right)$ für alle Bildpunkte innerhalb der markierten ROI und kalkuliert aus der durchschnittlichen Geschwindigkeit und der Fläche $\left(\mathrm{mm}^{2}\right)$ durch Multiplikation den resultierenden Fluss $\left(\mathrm{ml} \mathrm{s}^{-1}\right.$ ) entlang der Zeitserie (Abb. 5). Somit bezeichnen im Folgenden angegebene Geschwindigkeitswerte die durchschnittliche Flussgeschwindigkeit des Hirnwassers innerhalb der ROI.

Die Fläche unter der Kurve entspricht dem Flussvolumen (ml).

Abschließend gibt die Software die kalkulierten Werte (Maximal- und Minimalgeschwindigkeit, durchschnittliche Geschwindigkeit, Fläche, Fluss) mit der zeitlichen Auflösung von $125 \mathrm{~ms}$ in einem Auswertungsreport an. Dafür wird als Datenformat Excel (Microsoft Office Excel 2007) verwendet.

Jede einzelne forcierte In- und Exspiration wurde konsistent zur vorherigen Studie (DrehaKulaczewski et al. 2017) und unabhängig vom individuellen Flusskurvenverlauf standardisiert für jeden Probanden erfasst. Geringfügige individuelle Abweichungen von den vorgegebenen Zeiten des Atemprotokolls wurden ignoriert. 

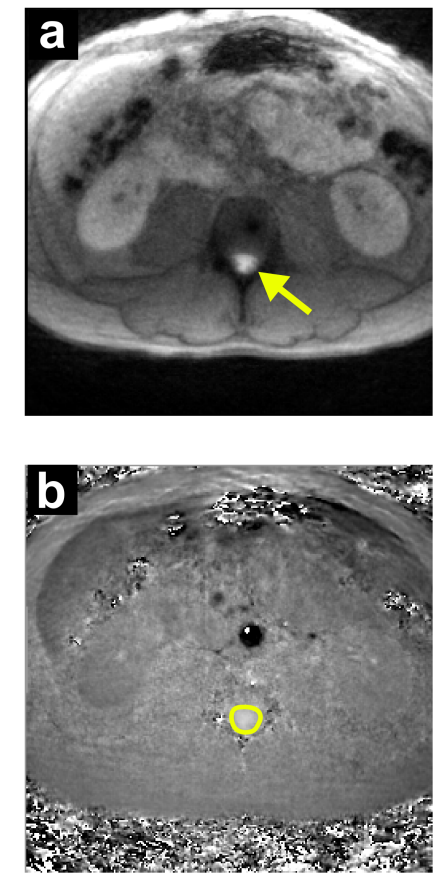
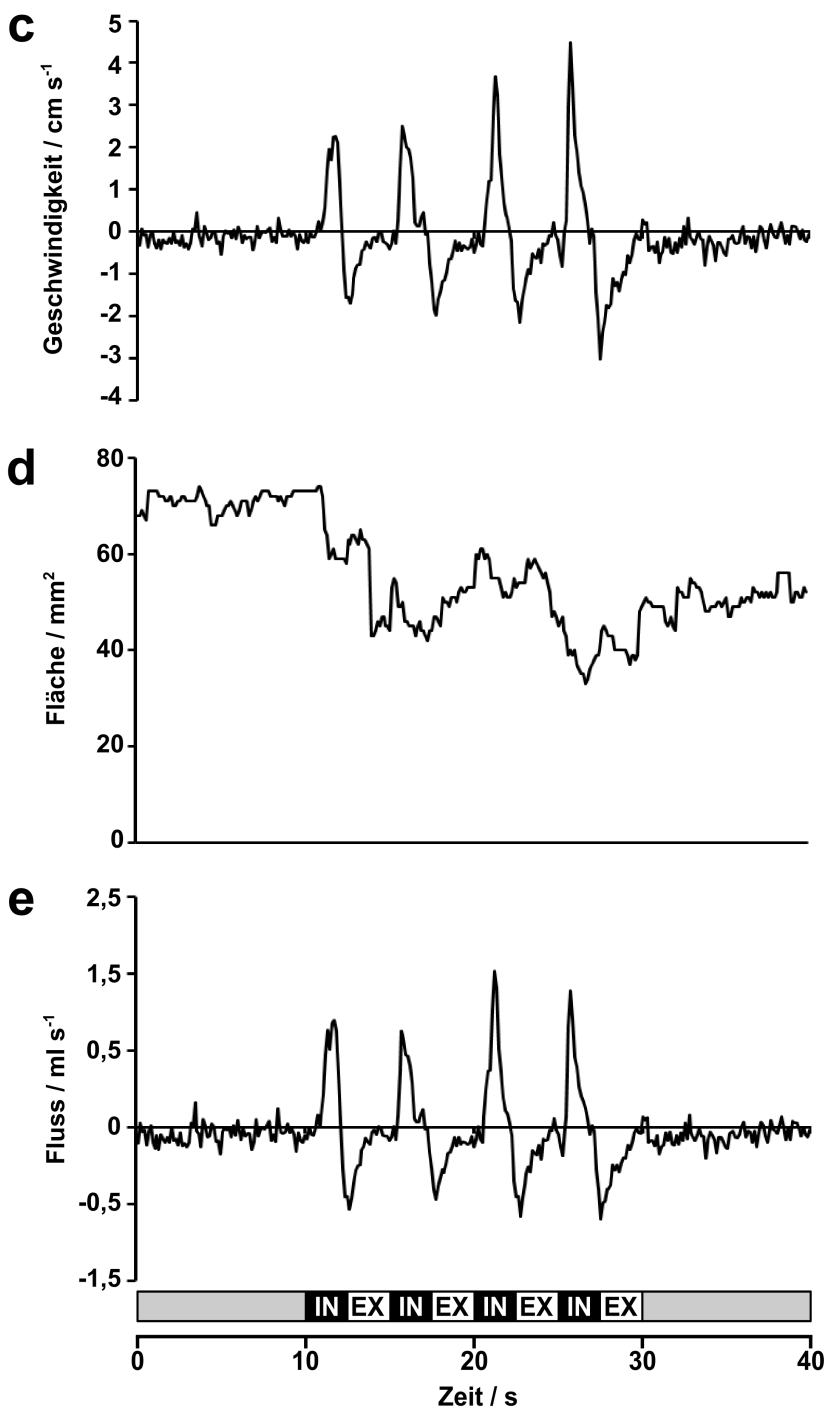

Abbildung 5: (a) Transversales Magnitudenbild (Pfeil markiert Hirnwasser im Spinalkanal) und (b) korrespondierendes Phasenbild mit eingezeichneter ROI (Signal hell = Flussrichtung nach kranial) einer Phasenkontrastflussmessung in Position L4 während forcierter Atmung. (c) Durchschnittliche Geschwindigkeit ( $\mathrm{cm} \mathrm{s}^{-1}$ ) innerhalb der ROI, (d) Fläche der ROI $\left(\mathrm{mm}^{2}\right)$ und (e) der daraus resultierende Fluss des Hirnwassers $\left(\mathrm{ml} \mathrm{s}^{-1}\right.$ ) entlang der Zeitserie (40 s) derselben Messung sowie das vorgegebene Atemprotokoll (IN: forcierte Inspiration, EX: forcierte Exspiration). 


\subsubsection{Korrelation der Thorax-/Bauchwandbewegung mit dem Zeitverlauf des Atemprotokolls}

Um sicherzustellen, dass die Probanden das Atemprotokoll zeitlich korrekt befolgten, wurden in einer definierten statischen ROI die Bewegungen der Thorax- bzw. Bauchwand und die daraus resultierenden Signalintensitätsänderungen erfasst und mit dem Zeitverlauf des Atemprotokolls verglichen.

Im Detail wurde mithilfe der CaFuR Software eine ROI auf dem Magnitudenbild so eingezeichnet, dass diese zum Zeitpunkt einer forcierten Ausatmung (Abb. 6a) und der damit einhergehenden Abflachung der Bauchwand neben Pixel (Bildpunkt) der Bauchwand auch darüberliegende Luft umfasst. Aufgrund der physiologischen Thorax- und Bauchwandbewegungen während der tiefen Atmung ändern sich die Anteile von Luft und Bauchwandgewebe innerhalb der ROI. Am Ende einer forcierten Einatmung mit maximaler Hebung der Bauchwand umfasst die ROI (Abb. 6b) ausschließlich Pixel der Bauchwand. Mittels der Software werden anschließend in dem definierten Bildbereich anhand der unterschiedlichen Graustufen der Pixel die Gesamtsignalintensitäten berechnet und gegen die Zeit aufgetragen (Abb. 6c).

Während der normalen Atmung fluktuieren die Signalintensitäten nur leicht. Während der forcierten Atmung steigt mit Beginn der tiefen Einatmung die Signalintensität deutlich an und sinkt mit der tiefen Ausatmung. Die Maxima des Kurvenverlaufs werden während der forcierten Inspiration erreicht. Hier stimmt die zeitliche Einhaltung des Atemprotokolls mit der Änderung der Signalintensität überein. Somit hat der Proband das Atemprotokoll korrekt befolgt. 


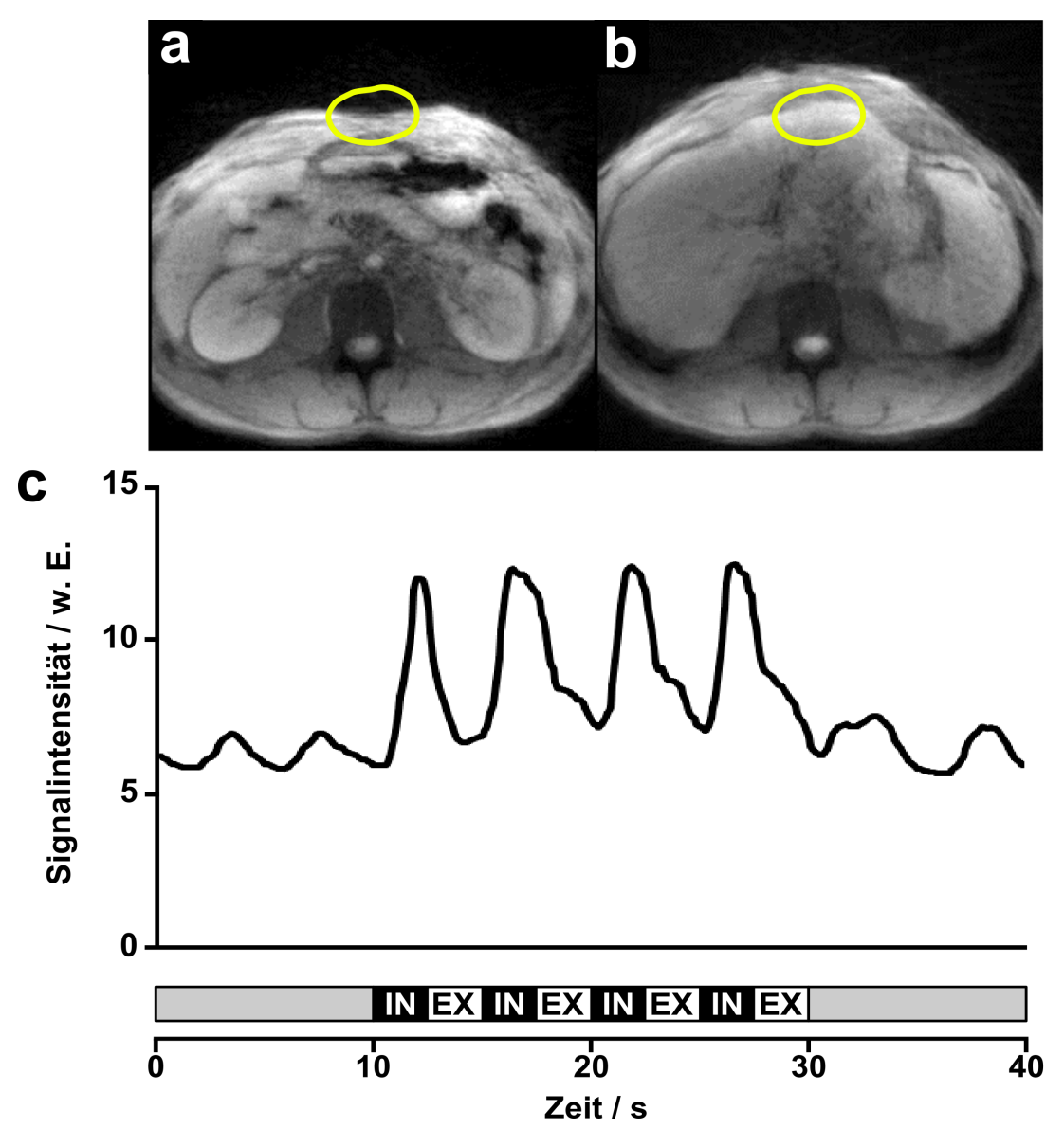

Abbildung 6: Magnitudenbild mit definierter ROI während (a) forcierter Exspiration und (b) forcierter Inspiration in Position L4. (c) Änderung der Signalintensität in der ROI über der Bauchwand (w. E.: willkürliche Einheiten) entlang der Zeitserie (40 s) in Position L4 sowie das vorgegebene Atemprotokoll (IN: forcierte Inspiration, EX: forcierte Exspiration).

\subsubsection{Berechnung der Flussvolumina des Hirnwassers}

Aus den individuellen Flusskurvenverläufen der einzelnen Probanden (s. Kapitel 3.1.1) wurde für jede Messposition zunächst das Flussvolumen des Hirnwassers während der forcierten Atmung (der Fluss integriert über die Zeit) berechnet. Entsprechend dem Atemprotokoll erhält man je vier Volumenwerte für die In- und Exspiration, d. h. Volumina, die innerhalb von 2,5 Sekunden fließen. Die Richtung des Flusses wird durch das Vorzeichen des Flussvolumens repräsentiert.

Darauf wurden die Mittelwerte über die vier Atemzyklen für jeweils die In- und Exspiration gebildet. Das Netto-Volumen während eines vollen forcierten Atemzyklus (5 Sekunden) pro Messposition für jeden Probanden ergibt sich aus der Summe (NET = IN + EX) der berechneten Mittelwerte (s. Kapitel 3.1.3). 
Als abschließender Schritt wurden die Mittelwerte über alle 19 Probanden für die Inspiration, Exspiration und Netto (s. Kapitel 3.1.4) kalkuliert.

\subsubsection{Signifikanztest}

Zur Überprüfung der Signifikanz wurde bei dem Vergleich der Gruppenmittel der in- und exspiratorischen sowie der Netto-Flussvolumina zwischen den einzelnen Messpositionen während der forcierten Atmung (s. Kapitel 3.1.4) ein einfaktorieller Anova-Signifikanztest in Kombination mit einem Post-Hoc-Test (Bonferroni-Korrektur; $p \leq 0,05$ ) durchgeführt.

\subsubsection{Tiefpassfilter}

In Anlehnung an die vorherige Studie (Dreha-Kulaczewski et al. 2017) wurde ein Tiefpassfilter (gemittelt über 9 Zeitpunkte) bei einigen zufällig ausgewählten Flusskurvenverläufen (s. Kapitel 3.1.1) angewandt. Dieser unterdrückt die höheren kardialen Frequenzen und lässt die tiefen passieren. Somit wird verdeutlicht, dass der Fluss des Hirnwassers direkt der Respiration folgt.

\subsubsection{Frequenzanalyse des Hirnwasserflusses}

Für die Darstellung des Einflusses der respiratorischen und kardialen Frequenzkomponenten auf den Fluss des Hirnwassers (s. Kapitel 3.2) wurden die Flussdaten während der forcierten Atmung zunächst Fourier transformiert. Infolgedessen entsteht aus dem zeitabhängigen Flusssignal durch die Zerlegung in Sinus- und Cosinusfunktionen ein frequenzabhängiges Signal (Flussspektrum). Um die Frequenzverteilungen über Positionen und Probanden hinweg vergleichbar zu machen, wurde jedes individuelle Frequenzspektrum auf ihren Mittelwert normiert. Für die Berechnung und Darstellung dieser Flussspektren (Fourier-transformierte Intensitäts-Zeitverläufe) wurde Matlab R2015a (Mathworks, USA) genutzt.

Um im Folgenden konkreter zwischen respiratorischen und kardialen Einfluss zu unterscheiden, wurden zwei Frequenzbereiche getrennt betrachtet. Für die Herzaktion wurden bei interindividuell schwankenden Herzfrequenzen, Pulsraten zwischen 48 und 108 Herzschläge/Minute angenommen. Dies entspricht einem Frequenzbereich von $0,8 \mathrm{~Hz}$ bis $1,8 \mathrm{~Hz}$. Frequenzkomponenten in diesem Bereich können im Wesentlichen auf die Herzaktivität zurückgeführt werden. Entsprechend einer Frequenz von $0,2 \mathrm{~Hz}$, beträgt 
die Periodendauer eines forcierten Atemzyklus 5 Sekunden, sodass Frequenzkomponenten in diesem Bereich auf den Einfluss der Atmung zurückzuführen sind. Für die genannten Frequenzbereiche wurden die Maximalwerte bestimmt und anschließend über alle Probanden gemittelt. Ein Vergleich des relativen Spektralanteils der Frequenzen bei $0,2 \mathrm{~Hz}$ und im Bereich von 0,8 bis $1,8 \mathrm{~Hz}$ an jeder Messposition ist nun möglich, sodass der kardiale Einfluss auf den Fluss des Hirnwassers im Gruppenmittel trotz individuell schwankender Herzfrequenzen direkt mit dem respiratorischen Einfluss verglichen werden kann. 


\section{Ergebnisse}

In den Ergebnissen werden zwei Aspekte der Flussdynamik des Hirnwassers betrachtet: zum einen, wieviel und in welche Richtung das Hirnwasser in den einzelnen Positionen entlang des Spinalkanals im Gruppenmittel während der forcierten Atmung fließt (s. Kapitel 3.1), zum anderen, welchen Anteil die Atmung und die Herzaktion jeweils an dem Fluss des Hirnwassers entlang des Spinalkanals im Gruppenmittel während der forcierten Atmung haben (s. Kapitel 3.2).

Insgesamt wurden die Daten von 200 (10 Positionen von 20 Probanden) Messserien erhoben. Die Daten eines Probanden (männlich, 26 Jahre) wurden retrospektiv von der Auswertung ausgeschlossen, da das Atemprotokoll nicht korrekt eingehalten wurde.

Von den verbliebenen 190 Messserien (10 Positionen von 19 Probanden) der Flussmessungen des Hirnwassers konnten insgesamt 18 Messserien (s. Kapitel 2.5) nicht berücksichtigt werden.

\subsection{Fluss und Volumen des Hirnwassers entlang des Spinalkanals}

Im Folgenden werden individuelle Flusskurven verschiedener Positionen gezeigt, die anschließend im Gruppenmittel zusammengefasst werden. Einerseits werden durch die individuellen Daten Unterschiede in den Positionen deutlich. Andererseits zeigen alle Probanden in den vorhandenen Messpositionen einen ähnlichen Trend. Die jeweiligen Amplituden können jedoch individuell variieren. Am Ende des Kapitels werden die inund exspiratorischen Volumina sowie die Netto-Flussvolumina im Gruppenmittel in Abhängigkeit von der Position dargestellt. 


\subsubsection{Individuelle Flusskurven des Hirnwassers}

Die Flusskurve eines jeden Probanden folgt präzise dem Atemprotokoll mit einem kranialen Fluss während der Inspiration und kaudalem Fluss während der Exspiration. Dies wird in Abb. 7 anhand einer konkreten Messung in Position L4 deutlich, wobei die Amplituden dieses Probanden in L4 höher ausfallen als bei anderen Probanden (s. Abb. 8 sowie A2 und A3, Anhang).
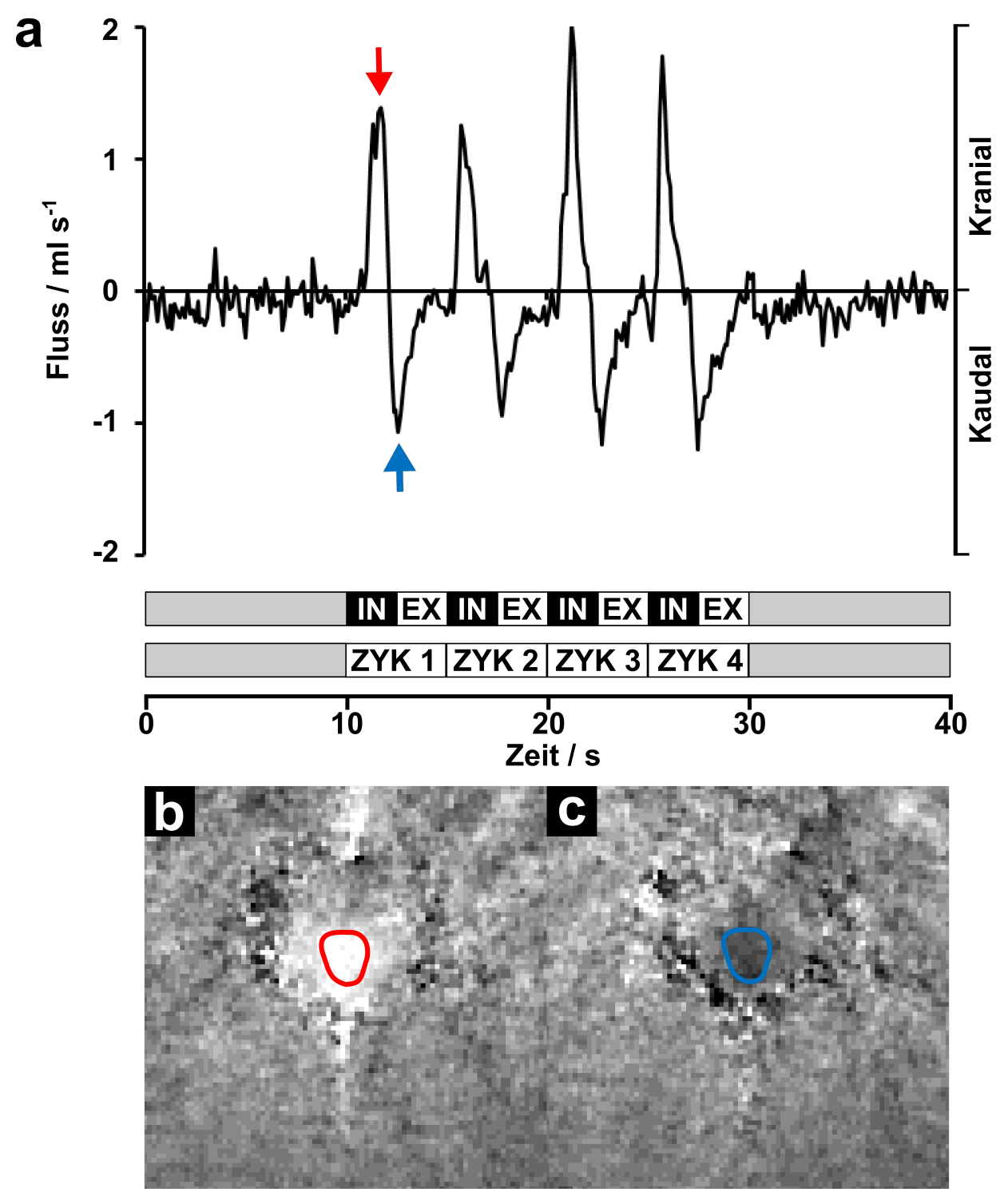

Abbildung 7: (a) Flusskurve des Hirnwassers $\left(\mathrm{ml} \mathrm{s}^{-1}\right.$ ) entlang der Zeitserie (40 s) in Position L4. Roter Pfeil $=$ forcierte Inspiration $=$ Flussrichtung nach kranial. Blauer Pfeil $=$ forcierte Exspiration = Flussrichtung nach kaudal. Über der Zeitachse ist das Atemprotokoll eingefügt (IN: forcierte Inspiration, EX: forcierte Exspiration, ZYK: Atemzyklus). (b) Transversales Phasendifferenzbild des Spinalkanals (Ausschnitt) während forcierter Inspiration $(\mathrm{ROI}$ rot $=$ Signal hell $=$ Flussrichtung nach kranial) und (c) forcierter Exspiration (ROI blau = Signal dunkel = Flussrichtung nach kaudal) einer Messserie in Position L4 zum selben Zeitpunkt wie die Flusskurve in (a). 
In den ersten 10 Sekunden der Messung (normale Atmung) schwankt die Flusskurve des Liquors (Abb. 7a) geringfügig um die Nullinie (Minimum $-0,4 \mathrm{ml} \mathrm{s}^{-1}$, Maximum $\left.0,3 \mathrm{ml} \mathrm{s}^{-1}\right)$. Mit Beginn der ersten tiefen Einatmung zeigt sich ein deutlicher Anstieg $\left(1,4 \mathrm{ml} \mathrm{s}^{-1}\right.$, Inspiration, 1. Atemzyklus, roter Pfeil, Abb. 7a) der Flusskurve. Alle positiven Werte entsprechen einem nach kranial gerichteten Fluss, kodiert mit einem hellen Signal (Abb. 7b). In der darauffolgenden forcierten Exspiration desselben Atemzyklus fällt die Flusskurve (-1,3 $\mathrm{ml} \mathrm{s}^{-1}$, Exspiration, 1. Atemzyklus, blauer Pfeil, Abb. 7a) unter die Nulllinie. Der negative Wert zeigt die kaudale Flussrichtung an (dunkles Signal, Abb. 7c).

Die Flussdynamik und -richtung ändert sich unmittelbar jeweils mit Beginn der In- und nachfolgenden Exspiration. Die Kurven folgen, wie in diesem Beispiel, den durch das Atemprotokoll vorgegebenen Atemzyklen. Maxima liegen während der Inspiration bei $1,1 \mathrm{ml} \mathrm{s}^{-1} \mathrm{im} \mathrm{2.} \mathrm{Atemzyklus,} \mathrm{bei} 2,0 \mathrm{ml} \mathrm{s}^{-1} \mathrm{im} \mathrm{3.} \mathrm{Atemzyklus} \mathrm{und} \mathrm{bei} 1,8 \mathrm{ml} \mathrm{s}^{-1} \mathrm{im} 4$. Atemzyklus. Minima werden bei der Exspiration $\left(-0,9 \mathrm{ml} \mathrm{s}^{-1} \mathrm{im} 2\right.$. Atemzyklus, $-1,2 \mathrm{ml} \mathrm{s}$ im 3. und 4. Atemzyklus) erreicht (Abb. 7a). In den letzten 10 Sekunden der Messung (normale Atmung) ist der Fluss gering (Minimum -0,4 $\mathrm{ml} \mathrm{s}^{-1}$, Maximum 0,2 $\mathrm{ml} \mathrm{s}^{-1}$ ).

Die Kurvenverläufe der Flussbewegung variieren zwischen den Messpositionen bezogen auf die Amplitude und das Verhältnis zwischen langsamen und schnellen Oszillationen (Abb. 8). Der Einfluss der Atmung auf die Flussbewegungen wird in der tiefpassgefilterten Darstellung (Abb. A1, Anhang) deutlich. 
C3

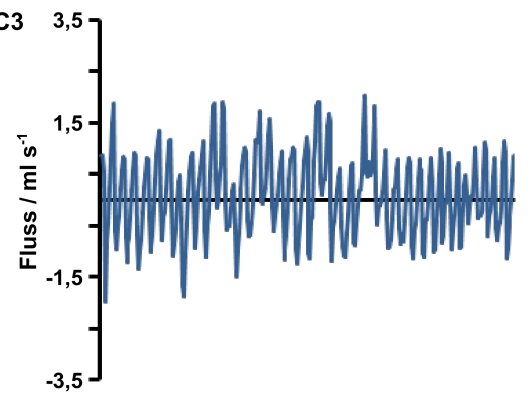

C5

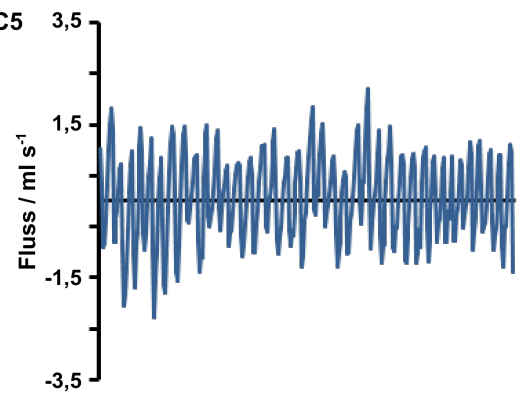

Th1

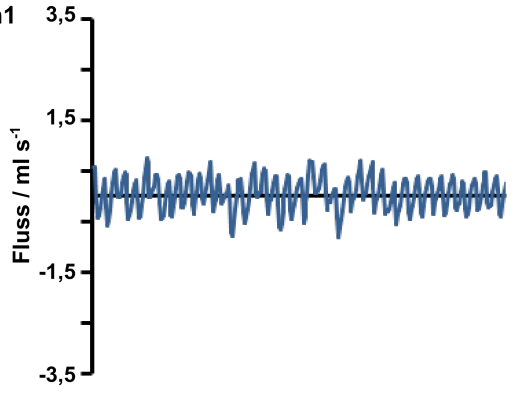

Th4

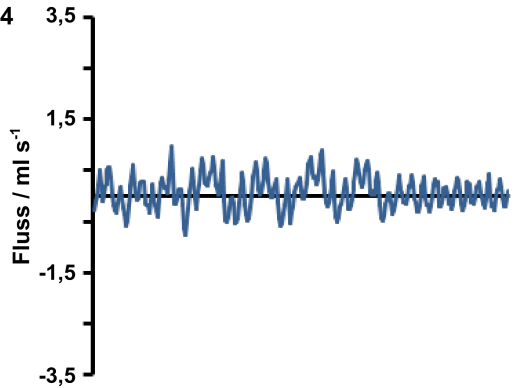

Th6

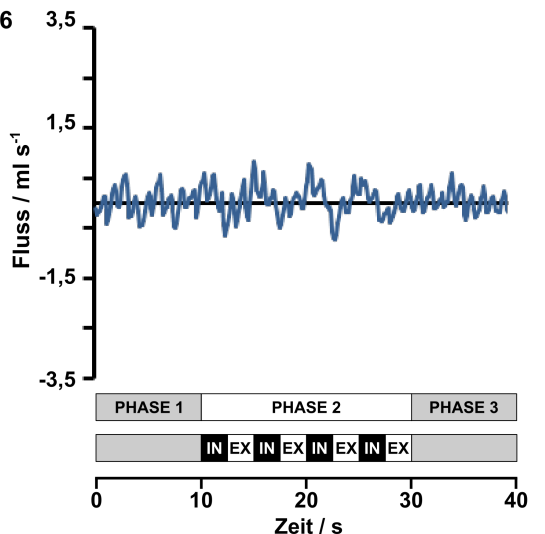

Th8

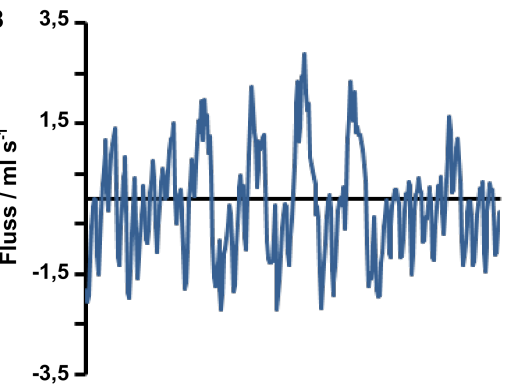

Th10

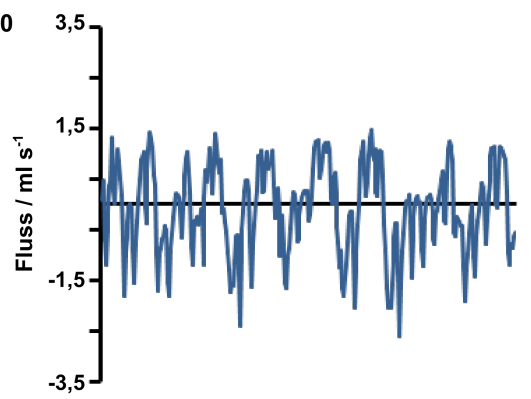

Th12
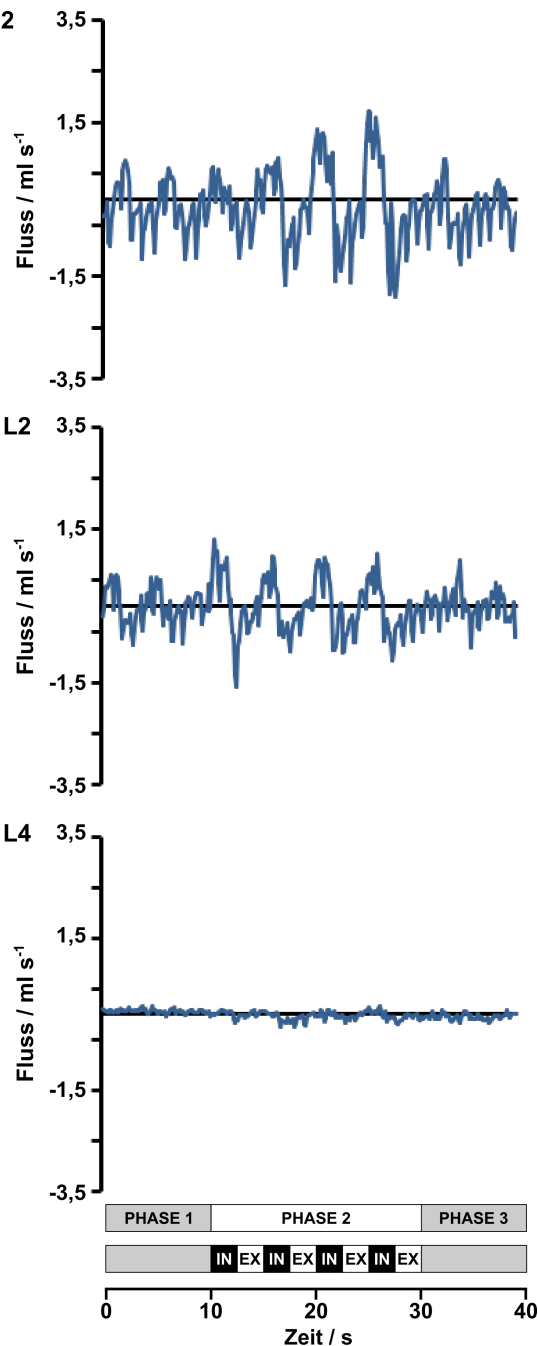

Abbildung 8: Flusskurven des Hirnwassers $\left(\mathrm{ml} \mathrm{s}^{-1}\right.$ ) entlang der Zeitserie (40 s) mit Atemprotokoll in allen Messpositionen des Probanden \#17 (Phase 1: normale Atmung, Phase 2: forcierte Atmung, Phase 3: normale Atmung, IN: forcierte Inspiration, EX: forcierte Exspiration). 
In den oberen Positionen C3 und C5 (Abb. 8) treten während der gesamten Messung schnelle pulsierende Flussänderungen auf, die auf die Herzaktion zurückzuführen sind. Die Flusswerte befinden sich in diesen Positionen im Bereich von $-2,3 \mathrm{ml} \mathrm{s}^{-1}$ bis 2,2 $\mathrm{ml} \mathrm{s}^{-1}$ und sind repräsentativ für andere Probanden (s. Abb. A2 und A3, Anhang). In den Positionen Th1, Th4 und Th6 überwiegen höherfrequente Flussänderungen. Hier liegt der Fluss im Bereich von $-0,8 \mathrm{ml} \mathrm{s}^{-1}$ bis $1,0 \mathrm{ml} \mathrm{s}^{-1}$ und ist somit niedriger im Vergleich zu dem Fluss in den oberen Positionen und in diesem Fall auch im Vergleich zu denselben Positionen anderer Probanden (s. Abb. A2 und A3, Anhang).

In den Positionen Th8, Th10, Th12 und L2 liegen die Flusswerte während Phase 1 und 3 (normale Atmung) im Bereich von $-2,0 \mathrm{ml} \mathrm{s}^{-1}$ bis $1,6 \mathrm{ml} \mathrm{s}^{-1}$ und sind vergleichbar mit den Flusswerten in den oberen Positionen C3 und C5. In Phase 2 (forcierte Atmung) erfolgt mit Beginn der forcierten Inspiration ein deutlicher Anstieg und mit tiefer Exspiration ein Abfall der Flusskurve. Die Werte liegen im Bereich von $-2,6 \mathrm{ml} \mathrm{s}^{-1}$ bis $2,4 \mathrm{ml} \mathrm{s}^{-1}$.

In der untersten Position (L4) ist der Fluss, verglichen mit den bereits beschriebenen Flusskurven, gering $\left(-0,2 \mathrm{ml} \mathrm{s}^{-1}\right.$ bis $\left.0,2 \mathrm{ml} \mathrm{s}^{-1}\right)$. Auch in den letztgenannten Positionen (Th8-L4) liegen die Werte, verglichen mit denen anderer Probanden, im Durchschnitt (s. Abb. A2 und A3, Anhang).

Vergleicht man die Flusskurvenverläufe zwischen den gleichen Positionen verschiedener Probanden (s. Abb. A2 und A3, Anhang), zeigen sich Unterschiede bezüglich Maximalamplitude und dem Verhältnis zwischen hoch- und niederfrequenten Schwingungen. Trotz individueller Unterschiede wird das Muster der prompten Antwort des Flusssignals auf die forcierte Atmung deutlich. 


\subsubsection{Geschwindigkeit, Fläche der ROI und Fluss des Hirnwassers im Gruppenmittel}

Das Gruppenmittel der Geschwindigkeit des Hirnwassers aller 19 Probanden (Abb. 9) variiert zwischen den Messpositionen und Atemphasen.

Unmittelbar mit Beginn der ersten forcierten Inspiration richtet sich der Fluss des Hirnwassers in allen Messpositionen nach kranial, und die Geschwindigkeit steigt (rote Farbkodierung), wie Abb. 9 verdeutlicht. Im thorakalen Bereich (Th6, Th8, Th10, Th12) werden dabei die höchsten Geschwindigkeiten in Richtung Kopf erreicht. In der darauffolgenden forcierten Exspiration alternieren in den Positionen C3, C5 und Th1 die Richtungen der Geschwindigkeiten (abwechselnd blau und rot). Im Bereich Th4 und abwärts ist die Geschwindigkeit hingegen strikt nach kaudal gerichtet (blau). Dabei werden in den Positionen Th6, Th8, Th10 und Th12 die höchsten negativen Geschwindigkeiten erreicht.

Während der normalen Atmung (Phase 1 und 3) alterniert die Geschwindigkeit in den Positionen C3, C5, Th1 und Th4 zwischen kranialer und kaudaler Richtung, wobei tendenziell der Fluss nach kranial dominiert. Kaudal von Th4 ist die Geschwindigkeit abwärts gerichtet. 


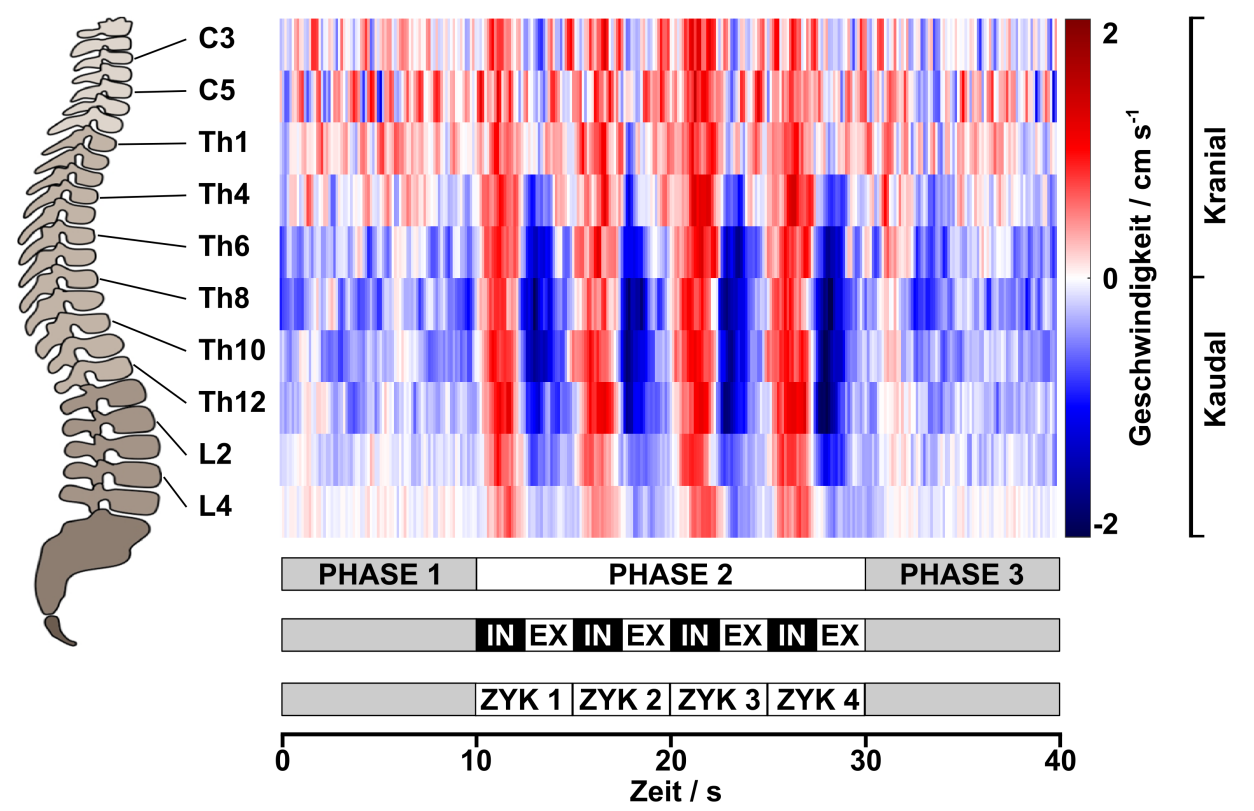

Abbildung 9: Geschwindigkeit des Hirnwassers $\left(\mathrm{cm} \mathrm{s}^{-1}\right)$ gemittelt über 19 Probanden für jede Messposition entlang der Zeitserie (40 s). Über der Zeitachse ist das Atemprotokoll eingefügt (Phase 1: normale Atmung, Phase 2: forcierte Atmung, Phase 3: normale Atmung, IN: Inspiration, EX: Exspiration, ZYK: Atemzyklus). Rechts: Farbkodierung der Geschwindigkeitswerte $\left(-2 \mathrm{~cm} \mathrm{~s}^{-1}\right.$ bis $\left.2 \mathrm{~cm} \mathrm{~s}^{-1}\right)$. Negative Werte (blau) entsprechen einem nach kaudal gerichteten Fluss. Positive Werte (rot) bedeuten einen Fluss des Hirnwassers nach kranial. Links: Wirbelsäule mit freundlicher Genehmigung von Dr. rer. nat. Stina Schiller, Universitätsmedizin Göttingen, 2018.

In Abb. 10 sind die Flächen der ausgewählten ROI im Gruppenmittel dargestellt. Im zervikalen Bereich $\left(\mathrm{C} 3, \mathrm{C} 5\right.$ ) beträgt die Fläche der ROI ca. 45 bis $55 \mathrm{~mm}^{2}$ (grünblaue Farbkodierung). In den Positionen Th1 (ca. 35 bis $45 \mathrm{~mm}^{2}$, blau), Th4 (ca. 25 bis $30 \mathrm{~mm}^{2}$, dunkelblau) und Th6 (ca. 30 bis $40 \mathrm{~mm}^{2}$, blau) nimmt diese ab. Bei Th4 ist die kleinste mittlere Fläche aller Messpositionen zu verzeichnen. Im unteren thorakalen Bereich (Th8 ca. 35 bis $45 \mathrm{~mm}^{2}$, blau /Th10 ca. 65 bis $70 \mathrm{~mm}^{2}$, grüngelb /Th12 ca. 55 bis $65 \mathrm{~mm}^{2}$, grün) nimmt die Fläche der ROI wieder zu. Bei Th10 wird das Maximum erreicht. Lumbal (L2, L4) beträgt die Fläche der ROI ca. 35 bis $45 \mathrm{~mm}^{2}$ (blau).

Innerhalb der einzelnen Positionen schwankt die Größe der Flächen entlang der Zeit - selbst während der forcierten Atmung - minimal. So hat beispielsweise die ROI in Position Th10 während der tiefen Ausatmung (Exspiration, 1. Atemzyklus) eine Fläche von ca. $67 \mathrm{~mm}^{2}$ (grüngelb) und während der normalen Atmung (Phase 3) eine Fläche von ca. $70 \mathrm{~mm}^{2}$ (gelb). 

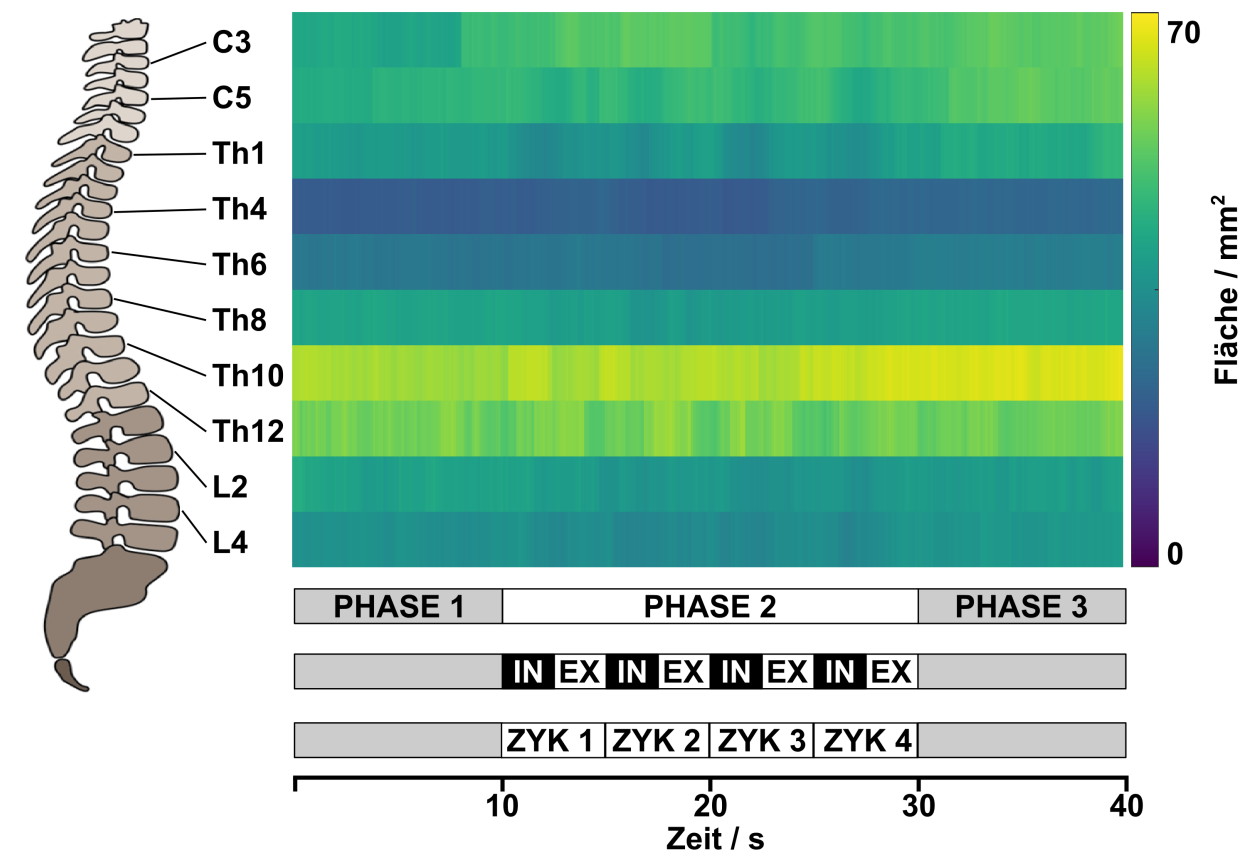

Abbildung 10: Fläche der ROI $\left(\mathrm{mm}^{2}\right)$ gemittelt über 19 Probanden für jede Messposition entlang der Zeitserie (40 s). Über der Zeitachse ist das Atemprotokoll eingefügt (Phase 1: normale Atmung, Phase 2: forcierte Atmung, Phase 3: normale Atmung, IN: Inspiration, EX: Exspiration, ZYK: Atemzyklus). Rechts: Farbkodierung der Flächengröße von $0 \mathrm{~mm}^{2}$ (violett) bis $70 \mathrm{~mm}^{2}$ (gelb). Links: Wirbelsäule mit freundlicher Genehmigung von Dr. rer. nat. Stina Schiller, Universitätsmedizin Göttingen, 2018.

Der gemittelte Fluss des Hirnwassers (Abb. 11), d. h. das Produkt aus mittlerer Geschwindigkeit (Abb. 9) und der jeweiligen mittleren Fläche der ROI (Abb. 10) unterscheidet sich qualitativ nur wenig von der mittleren Geschwindigkeit, da die ROI aufgrund ihrer zeitlichen Konstanz kaum einen Einfluss auf den zeitlichen Verlauf der Flusskurve hat.

Während der forcierten Einatmungen (Abb. 11) dominiert entlang aller Messpositionen (C3 bis L4) ein nach kranial gerichteter Fluss (rote Farbkodierung). Dabei ist der stärkste Fluss nach oben in den Positionen Th10 und Th12 zu verzeichnen. Während der forcierten Ausatmung alternieren in den oberen Positionen (C3, C5, Th1) auf- und abwärts (rot und blau) gerichtete Flusswerte. Weiter distal entlang des Spinalkanals (Th4 bis L4) zeigt das Hirnwasser überwiegend eine Bewegung nach kaudal (blau). Dabei fließt das Hirnwasser in den Positionen Th8, Th10 und Th12 am stärksten nach kaudal. 
In der normalen Atemphase (Phase 1 und 3) alterniert mit der Herzfrequenz die Flussrichtung in den oberen Positionen (C3, C5, Th1) zwischen kaudal und kranial (blau und rot). Tendenziell überwiegt jedoch der Fluss nach kranial (rot). In den weiteren thorakalen und lumbalen Positionen (Th4, Th6, Th8, Th10, Th12, L2) fließt das Hirnwasser abwärts (blau). Der stärkste Fluss nach kaudal erfolgt dabei in den Positionen Th8, Th10 und Th12. Im unteren lumbalen Bereich (L4) ist der Fluss des Hirnwassers gering (weiße Farbkodierung).

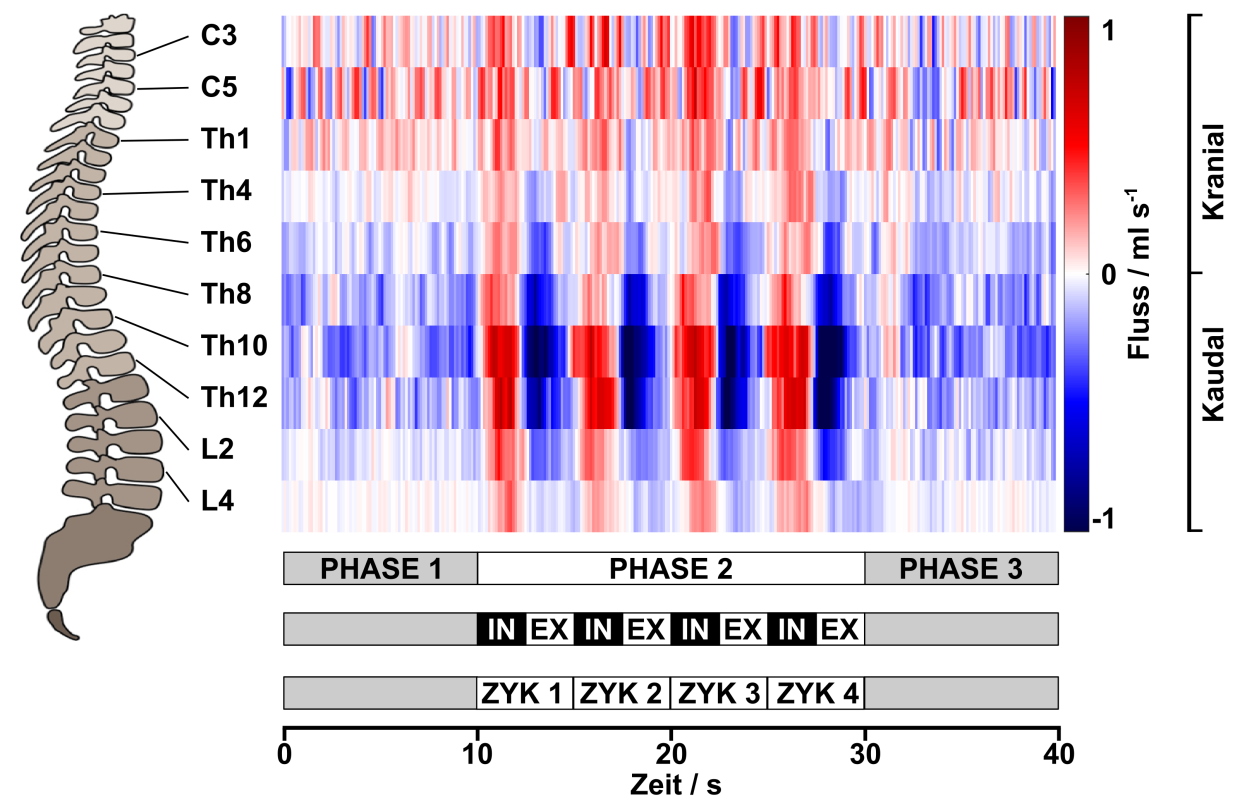

Abbildung 11: Fluss des Hirnwassers $\left(\mathrm{ml} \mathrm{s}^{-1}\right)$ gemittelt über 19 Probanden für jede Messposition entlang der Zeitserie (40 s). Über der Zeitachse ist das Atemprotokoll eingefügt (Phase 1: normale Atmung, Phase 2: forcierte Atmung, Phase 3: normale Atmung, IN: Inspiration, EX: Exspiration, ZYK: Atemzyklus). Rechts: Farbkodierung der Flusswerte $\left(-1 \mathrm{ml} \mathrm{s}^{-1}\right.$ bis $\left.1 \mathrm{ml} \mathrm{s}^{-1}\right)$. Negative Werte (blau) entsprechen einem nach kaudal gerichteten Fluss. Positive Werte (rot) bedeuten einen Fluss des Hirnwassers nach kranial. Links: Wirbelsäule mit freundlicher Genehmigung von Dr. rer. nat. Stina Schiller, Universitätsmedizin Göttingen, 2018. 


\subsubsection{Individuelle Volumenverschiebung des Hirnwassers während forcierter Atmung}

Nachdem in Abb. 8 (s. Kapitel 3.1.1) sowie in Abb. A2, A3 (Anhang) die individuellen Flusskurven des Hirnwassers während der gesamten Zeitserie betrachtet wurden, zeigt Abb. 12 die Volumenverschiebungen des Hirnwassers zweier exemplarisch ausgewählter Probanden (\#17, \#1) während der forcierten Atmung, wie in Kapitel 2.6.3 beschrieben. Die Werte für die in- und exspiratorischen Volumina sowie für die NettoFlussvolumina des Hirnwassers im Mittel über die Zyklen während der forcierten Atmung sind Tab. A2 (Anhang) zu entnehmen.

Während der forcierten Inspiration werden positive Flussvolumina (Fluss nach kranial) entlang des gesamten Spinalkanals registriert. Bei tiefer Ausatmung sind überwiegend negative Flussvolumina (Fluss nach kaudal) zu verzeichnen. Die Flussvolumina variieren während der Exspiration zwischen den Probanden stärker als während der Inspiration (Abb. 12a und c).

Während der Inspiration werden minimale Volumina in Position L4 (0,0 ml in Abb. 12a bzw. 0,1 $\mathrm{ml}$ in Abb. 12c) erreicht. Die maximalen Werte liegen im unteren thorakalen Bereich in Position Th8 (2,2 ml in Abb. 12a) bzw. Th10 (2,2 ml in Abb. 12c).

Minimale exspiratorische Volumina finden sich in den oberen zervikalen (C3: $-0,2 \mathrm{ml}$ in Abb. 12a bzw. $-0,3 \mathrm{ml}$ in Abb. 12c) und in den unteren lumbalen Messpositionen (L4: $-0,2 \mathrm{ml}$ in Abb. 12a bzw. $-0,3 \mathrm{ml}$ in Abb. 12c). Die maximalen exspiratorischen Volumina (Th8: $-2,3 \mathrm{ml}$ in Abb. 12a bzw. Th10: $-4,2 \mathrm{ml}$ in Abb. 12c) liegen im unteren thorakalen Bereich.

Die Netto-Volumina (Abb. 12b und d) sind im kranialen Bereich (C3 bis Th6) positiv (Fluss nach kranial). Unterhalb Th6 sind die Netto-Volumina hingegen negativ, einhergehend mit einem nach unten gerichteten Fluss.

Die individuellen Werte stehen im Einklang mit den entsprechenden Werten gemittelt über alle Probanden (s. Kapitel 3.1.4). 

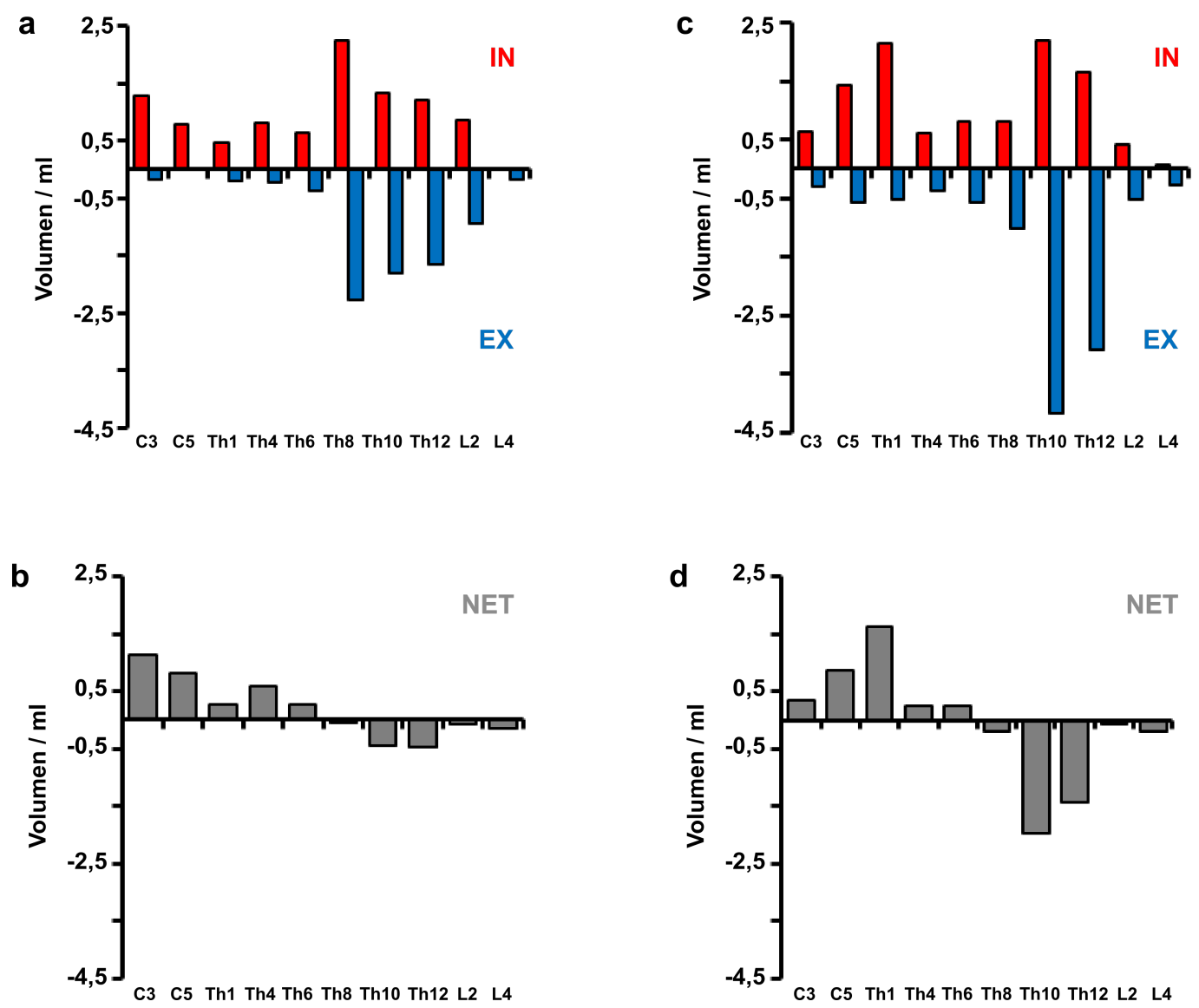

Abbildung 12: (a und c) In- und exspiratorische (IN = rot, EX = blau) Volumina des Hirnwassers im Mittel über die Atemzyklen während forcierter Atmung in allen Messpositionen der Probanden \#17 und \#1 (s. Kapitel 3.1.1, Abb. 8 sowie Abb. A2, Anhang). (b und d) Korrespondierende NettoVolumina (NET = grau) des Hirnwassers (\#17 a und b, \#1 c und d). Werte siehe Tab. A2 (Anhang).

\subsubsection{Gruppenmittel der Volumenverschiebung des Hirnwassers wäh- rend forcierter Atmung}

Das über alle 19 Probanden gemittelte in- und exspiratorische Volumen und das NettoFlussvolumen jeder Position ist in Abb. 13 aufgezeigt. Detaillierte Informationen über die berechneten Durchschnittswerte, gemittelt über alle Probanden (s. Kapitel 2.6.3) sowie die Standardabweichungen der in- und exspiratorischen Volumina und Netto-Volumina während der forcierten Atmung, sind Tab. A2 (Anhang) zu entnehmen. Signifikante Unterschiede ( $p$-Wert $\leq$ 0,05, s. Kapitel 2.6.4) der berechneten Volumina im Gruppenmittel zwischen den einzelnen Messpositionen während der forcierten Atmung zeigt Tab. A3 (Anhang). 
Während der forcierten Atmung sind die über alle Probanden gemittelten Volumina entlang des gesamten Spinalkanals ausschließlich positiv (Fluss nach kranial), wie in Abb. 13a gezeigt. Das Flussvolumen aller Positionen liegt während der forcierten Inspiration im Bereich von 0,3 $\mathrm{ml}$ bis $1,1 \mathrm{ml}$ bezogen auf 2,5 Sekunden. In Übereinstimmung mit den Werten der exemplarisch gezeigten Probanden \#17 und \#1 (s. Kapitel 3.1.3, Abb. 12a und c) wird im unteren thorakalen Bereich das maximale inspiratorische Volumen erreicht. Im Gruppenmittel liegt das maximale Flussvolumen hier in Position Th10 (Th10: 1,1 ml). Das minimale Flussvolumen während der Inspiration der ausgewählten Probanden (s. Kapitel 3.1.3, Abb. 12a und c) liegt genau wie beim Gruppenmittel im kaudalen lumbalen Bereich (L4: 0,3 ml).

Mit der forcierten Exspiration fließt das Hirnwasser im Gruppenmittel überwiegend nach kaudal (Abb. 13a). Die exspiratorischen Flussvolumina sind in den zervikalen und oberen thorakalen (C3 bis Th1: ca. 0,2 ml) Positionen gering und in der Richtung variabel. Im unteren thorakalen Bereich (Th8 bis Th12: $-0,9$ bis $-1,7 \mathrm{ml}$ ) zeigt sich ein deutlicher Fluss nach kaudal (s. Kapitel 3.1.3, Abb. 12a und c).

Aus den in- und exspiratorischen Flussvolumina resultieren oberhalb von Th4 positive Netto-Flussvolumina (bezogen auf 5 Sekunden Zyklusdauer) während der forcierten Atmung (Abb. 13b). Hier erfolgt der Fluss demzufolge nach kranial. Unterhalb von Th6 hingegen werden überwiegend negative Netto-Volumina gemessen. In diesen Abschnitten des Spinalkanals fließt das Hirnwasser während der forcierten Atmung vornehmlich nach kaudal. Im Einklang mit den Ergebnissen der individuellen Probanden (s. Kapitel 3.1.3, Abb. 12b und d) zeigt sich im Gruppenmittel eine Änderung der Flussrichtung im Bereich Th4-Th6. 

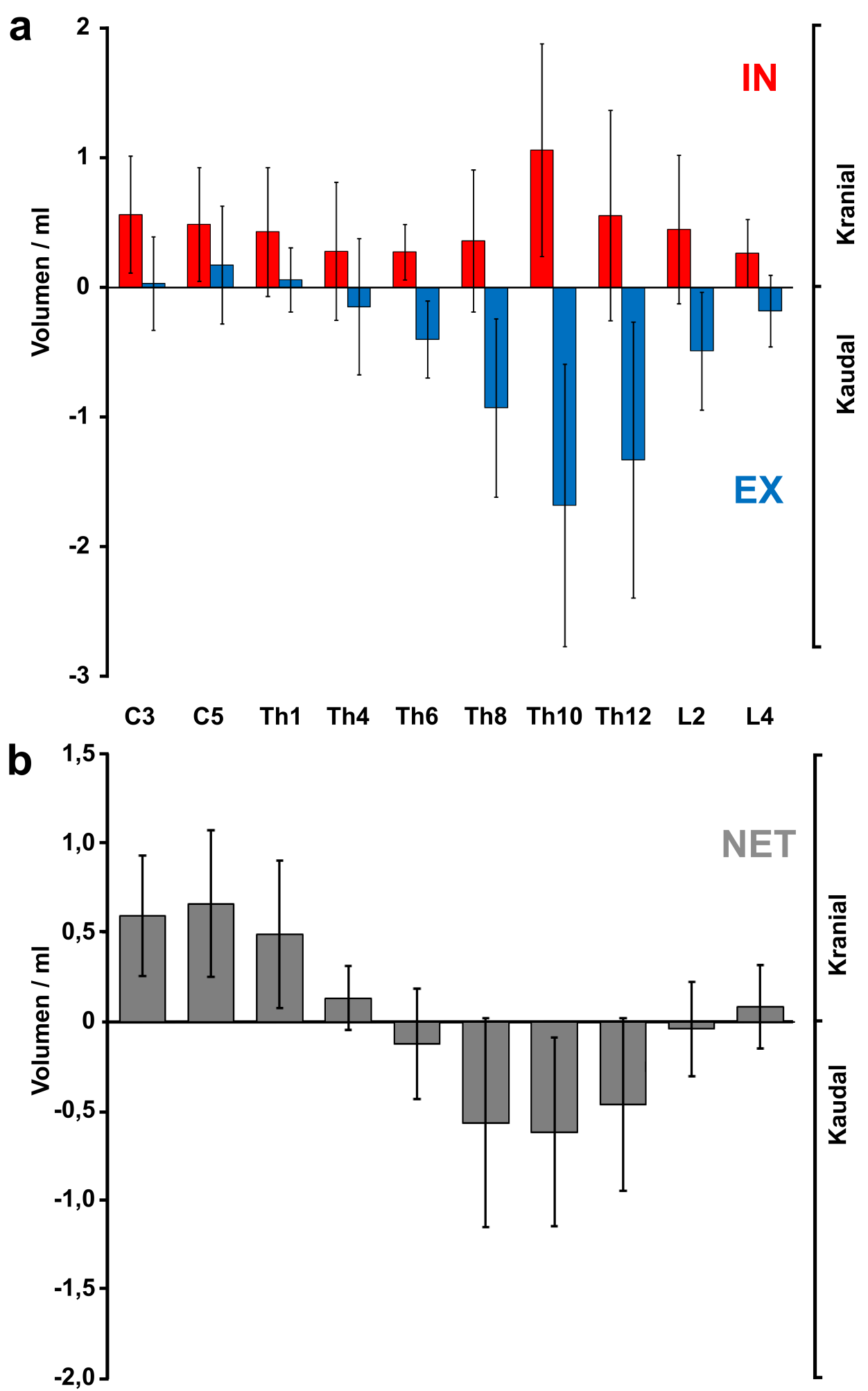

Abbildung 13: (a) In- und exspiratorische Volumina (IN = rot, EX = blau) des Hirnwassers bezogen auf 2,5 Sekunden Zyklusdauer und (b) des Netto-Volumens (NET = grau) bezogen auf 5 Sekunden Zyklusdauer gemittelt über 19 Probanden während forcierter Atmung in allen Messpositionen. Werte siehe Tab. A2 (Anhang). Die Fehlerbalken repräsentieren die Standardabweichung. 


\subsection{Anteil der respiratorischen und kardialen Frequenz- komponenten am Fluss des Hirnwassers während forcierter Atmung}

Nach der Betrachtung der Flussvolumina des Hirnwassers in den einzelnen Positionen soll nun erörtert werden, welchen Einfluss die forcierte Atmung und die Herzaktion auf die Dynamik des Hirnwassers in Abhängigkeit von der Lokalisation im Spinalkanal haben. Dazu wurde eine Frequenzanalyse (s. Kapitel 2.6.6) durchgeführt, d. h. der 20-sekündige Zeitverlauf Fourier transformiert.

Zur Verdeutlichung des respiratorischen und kardialen Einflusses auf das Flusssignal an einer kranialen und kaudalen Messposition, werden beispielhaft die Frequenzspektren des Flusssignals in Position C5 (Abb. 14a) und Th12 (Abb. 14b) des Probanden \#17 gezeigt.

In Position C5 (Abb.14a) liegt der maximale Ausschlag der Amplitude bei 0,9 Hz, sodass diese Flussbewegung hauptsächlich auf die Herzaktivität (54 Herzschläge/Minute) zurückzuführen ist. Bei der doppelten Frequenz von 1,8 Hz liegt ein weiteres Maximum, das als zweite Harmonische bezeichnet wird. Das Maximum bei 0,2 Hz spiegelt die respiratorische Frequenzkomponente wider. Die höchste relative Intensität thorakal (Abb. 14b) liegt bei $0,2 \mathrm{~Hz}$ - der Frequenzkomponente der forcierten Atmung. Ein weiteres Maximum ist bei $0,9 \mathrm{~Hz}$ (54 Herzschläge/Minute), entsprechend der Herzfrequenz, zu verzeichnen. 

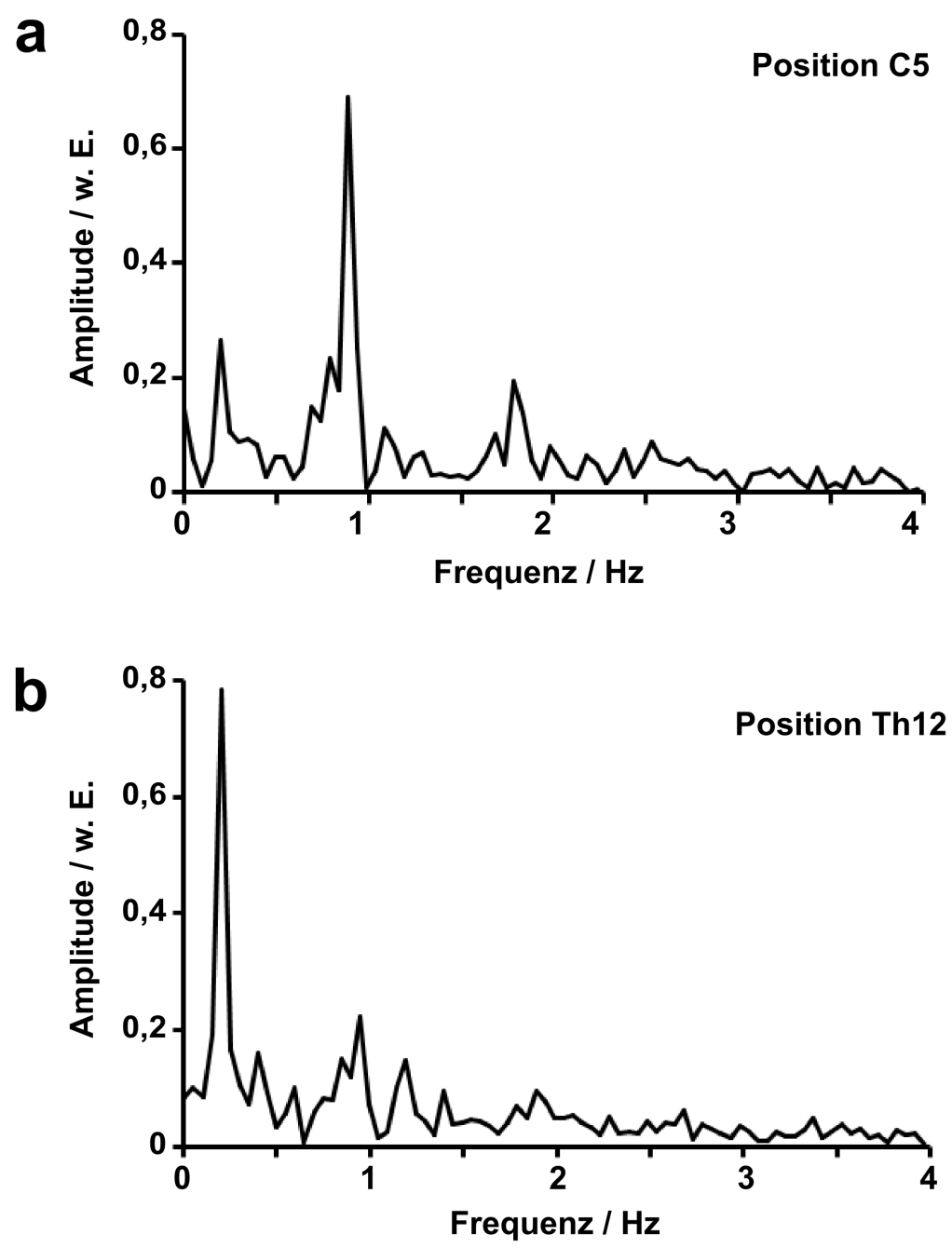

Abbildung 14: Frequenzspektrum (Proband \#17) des Flusssignals des Hirnwassers in den Positionen (a) C5 und (b) Th12. Die Amplitude des Fourier-transformierten Flusssignals (w. E.: willkürliche Einheiten) ist gegen die Frequenz $(\mathrm{Hz})$ aufgetragen. Die höchste relative Intensität in Position C5 liegt bei $0,2 \mathrm{~Hz}$ - entsprechend der Atemfrequenz. Die höchste relative Intensität in Position Th12 liegt bei $0,9 \mathrm{~Hz}$ - entsprechend der Herzfrequenz.

In Abb. 15 sind die Flussspektren der zehn Messpositionen eines Probanden (\#17) während der forcierten Atmung illustriert. Die Beschreibung erfolgt von kranial nach kaudal. In den oberen Messpositionen (C3, C5, Th1) liegt die höchste relative Intensität bei ca. $0,9 \mathrm{~Hz}$ (blaugrüne Farbkodierung), was durch die kardialen Frequenzkomponenten bedingt ist. Außerdem ist eine respiratorische Frequenzkomponente $(0,2 \mathrm{~Hz}$; blau) auf gleicher Höhe vorhanden. Hervorgerufen durch den 5-sekündigen Atemzyklus, dominiert unterhalb von Th4 eine Flussbewegung mit einer Frequenz von 0,2 Hz (grün bis gelb). An Position Th8 ist die respiratorische Frequenzkomponente auf die Flussbewegung des Liquors am stärksten (gelb). In allen genannten Messpositionen sind zudem Flussbewegungen mit einer Frequenz von ca. $1 \mathrm{~Hz}$ (Herzschlag; blau) zu finden. 


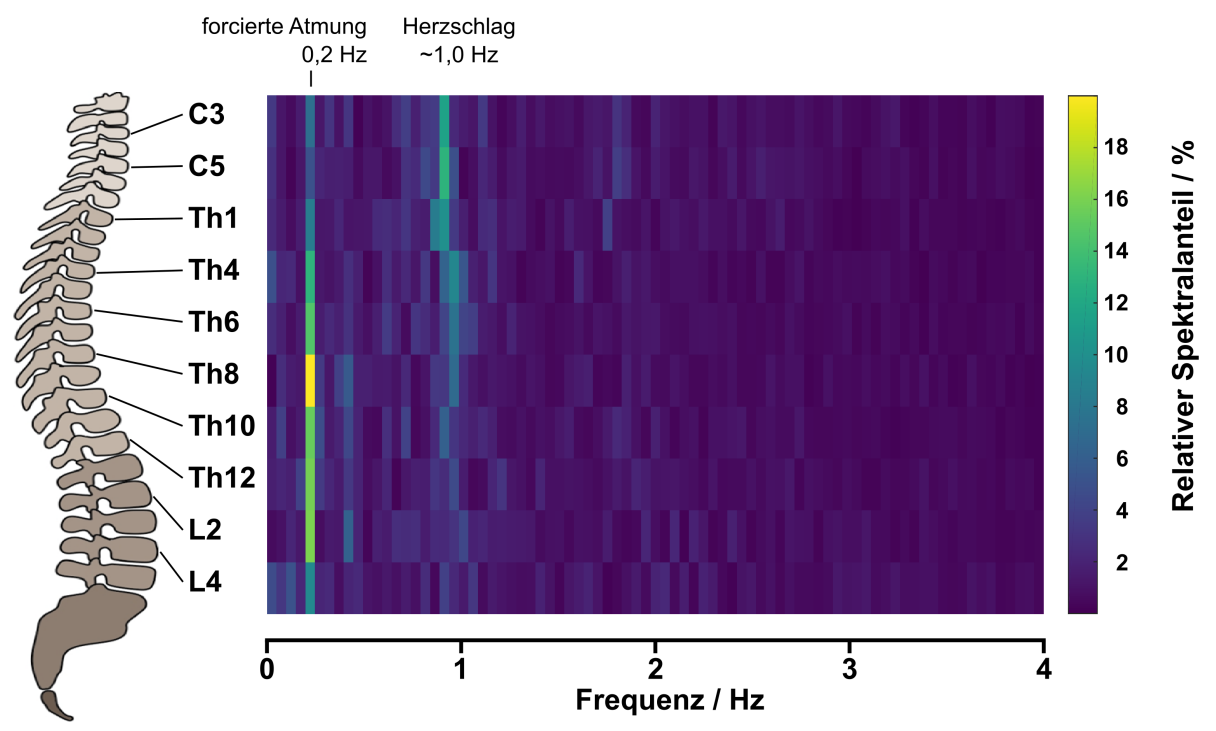

Abbildung 15: Frequenzspektren des Flusssignals des Hirnwassers in allen Messpositionen im Frequenzbereich von 0 bis $4 \mathrm{~Hz}$ des Probanden \#17; spektrale Auflösung $0,05 \mathrm{~Hz}$ entsprechend einer Abtastung der 20-sekündigen forcierten Atmung in 125 ms Schritten. Rechts: Farbkodierung des relativen Spektralanteils in \% (0\% bis $20 \%)$. Violett (0\% relativer Spektralanteil) kodiert für einen niedrigen und gelb (20\% relativer Spektralanteil) für einen hohen Anteil. Deutlich wird der jeweils positionsabhängige respiratorische Einfluss bei $0,2 \mathrm{~Hz}$ und der kardiale Einfluss bei ca. $1,0 \mathrm{~Hz}$. Alle Werte wurden so normiert, dass die Summe entlang einer Position $100 \%$ ergibt. Links: Wirbelsäule mit freundlicher Genehmigung von Dr. rer. nat. Stina Schiller, Universitätsmedizin Göttingen, 2018.

Die in Abb. 15 für einen Probanden gezeigte Frequenzverteilung verhält sich wie das in Abb. 16 dargestellte Gruppenmittel über alle Probanden während der forcierten Atmung. Aufgrund der interindividuell schwankenden Pulsraten sind die Frequenzkomponenten, die dem Herzschlag zuzuordnen sind, im Bereich von 0,8 bis $1,8 \mathrm{~Hz}$ verteilt - im Gegensatz zu der durch das Atemprotokoll vorgegebenen Atemfrequenz von 0,2 Hz.

Bei Betrachtung der Abb. 16 sind in den Positionen C3, C5 und Th1 Flussbewegungen mit Frequenzen von 0,2 $\mathrm{Hz}$ (respiratorische Frequenzkomponente; erste Harmonische; hellblau), von 0,4 Hz (zweite Harmonische; blau), von 0,6 Hz (dritte Harmonische; blau) sowie Frequenzen im Bereich von 0,8-1,6 Hz (blau) vertreten. Die letztgenannten Frequenzen können entsprechend 48 bis 96 Herzschläge/Minute auf die Herzaktivität zurückgeführt werden. 
Kaudal von Position Th4 dominiert die respiratorische Frequenzkomponente von $0,2 \mathrm{~Hz}$ (grün bis gelb). Die stärkste Intensität der Flussbewegung liegt bei Th10 und Th12 (gelb). Darüber hinaus treten auch kaudal von Th4 Frequenzen von ca. 0,4 Hz (zweite Harmonische; blau) sowie von ca. 0,6 Hz (dritte Harmonische; blau) auf. Bewegungen im Frequenzbereich von $0,8 \mathrm{~Hz}$ bis $1,6 \mathrm{~Hz}$ (blau) konnten in den Positionen Th4, Th6, Th8 und Th10 nachgewiesen werden und sind auf den Herzschlag zurückzuführen.

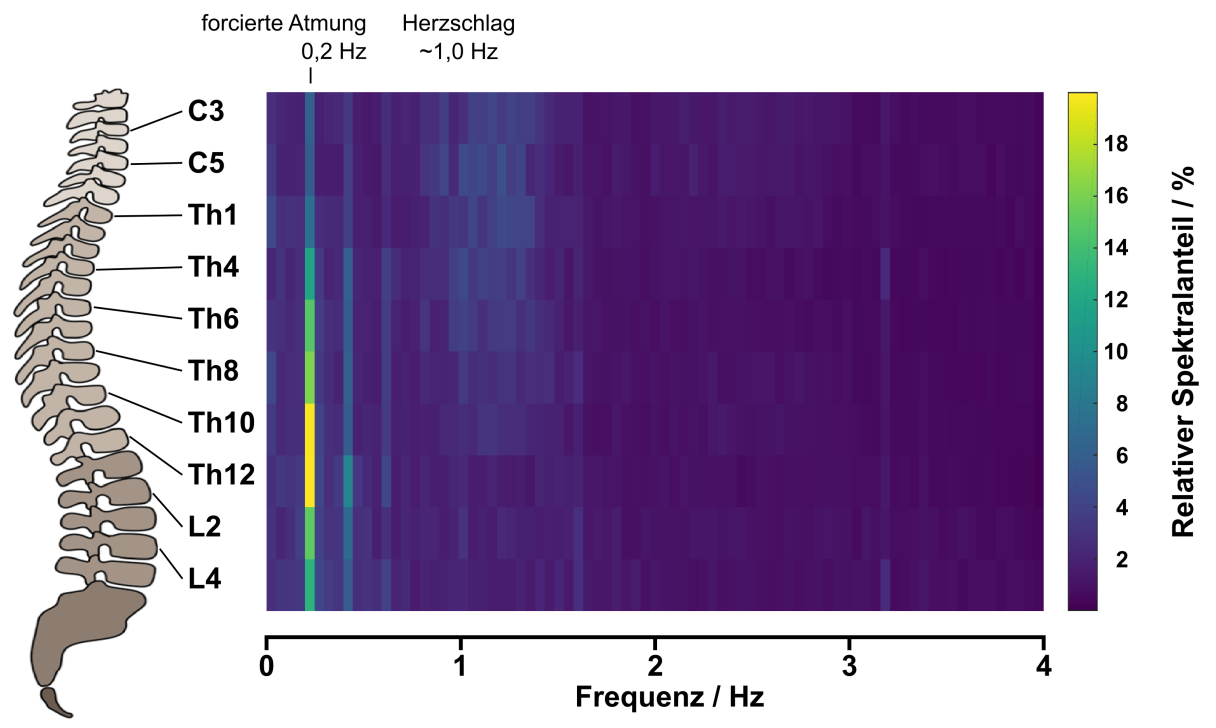

Abbildung 16: Frequenzspektren des Flusssignals des Hirnwassers in allen Messpositionen im Frequenzbereich von 0 bis $4 \mathrm{~Hz}$ gemittelt über 19 Probanden; spektrale Auflösung $0,05 \mathrm{~Hz}$ entsprechend einer Abtastung der 20-sekündigen forcierten Atmung in 125 ms Schritten. Rechts: Farbkodierung des relativen Spektralanteils in \% (0\% bis $20 \%$ ). Violett (0\% relativer Spektralanteil) kodiert für einen niedrigen und gelb (20\% relativer Spektralanteil) für einen hohen Anteil. Im Gegensatz zur Respirationsfrequenz unterliegt die Herzfrequenz interindividuellen Schwankungen, wodurch das Mittel verschmiert. Alle Werte wurden so normiert, dass die Summe entlang einer Position 100 \% ergibt. Links: Wirbelsäule mit freundlicher Genehmigung von Dr. rer. nat. Stina Schiller, Universitätsmedizin Göttingen, 2018.

Um den kardialen Einfluss auf den Fluss des Hirnwassers im Gruppenmittel trotz individuell schwankender Herzfrequenzen (Abb. 16) direkt mit dem respiratorischen Einfluss vergleichen zu können, ist in Abb. 17 der relative Spektralanteil der Atmung und der Herzaktion, wie in Kapitel 2.6.6 beschrieben, dargestellt. 

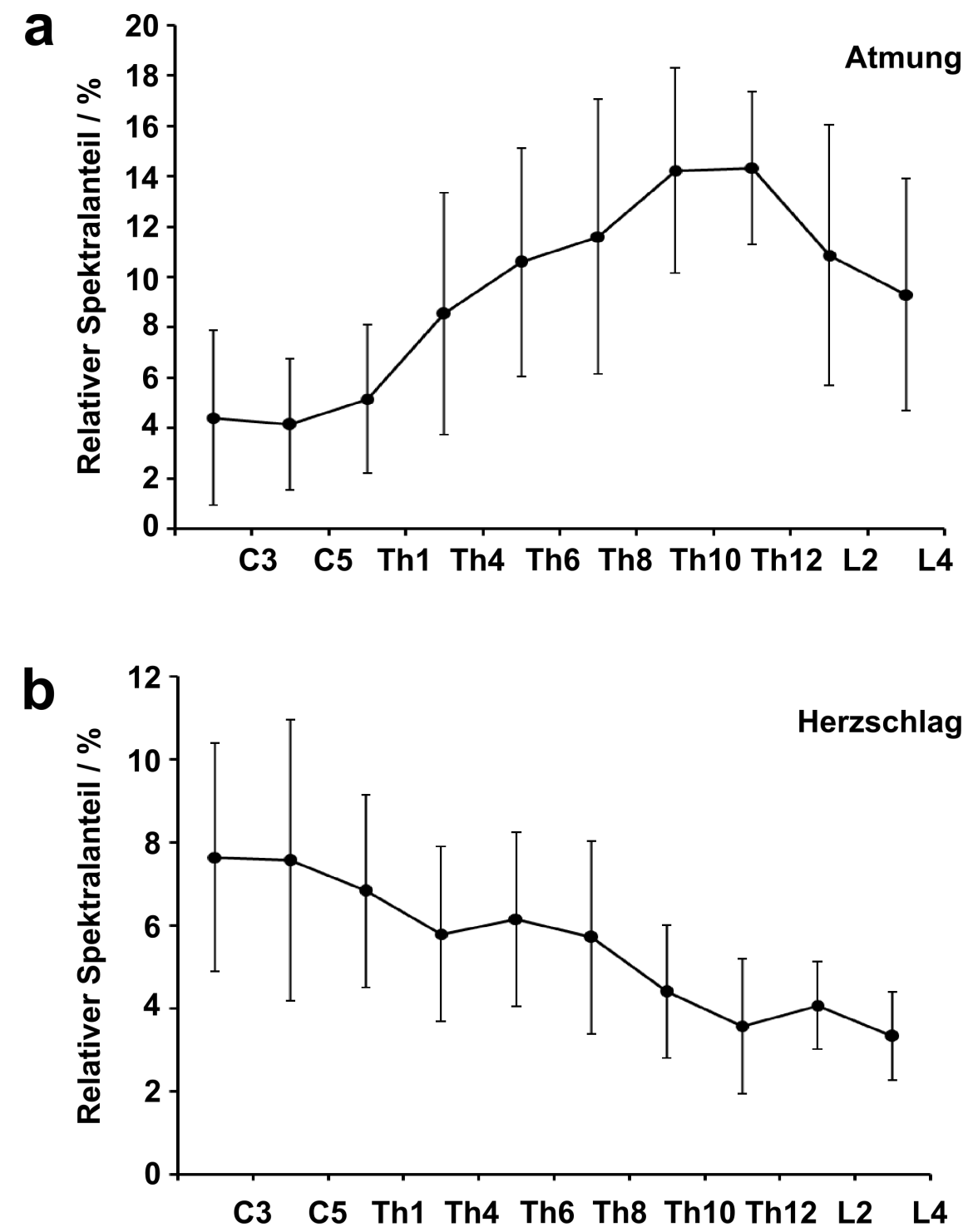

Abbildung 17: Anteil der (a) respiratorischen Frequenz von $0,2 \mathrm{~Hz}$ und (b) kardialen Frequenzen (jeweils Maximum im Bereich von 0,8 bis $1,8 \mathrm{~Hz}$ ) relativ zur Summe aller Frequenzen in Abhängigkeit der Messpositionen (s. Kapitel 2.6.6). Dargestellt sind die gemittelten Werte über 19 Probanden und die Standardabweichung als Fehlerbalken. Werte siehe Tab. A4 und A5 (Anhang).

In den zervikalen Positionen C3 und C5 ist der relative respiratorische Spektralanteil während der forcierten Atmung (Abb.17a) gering (4,4 \% in C3; 4,1 \% in C5). Dieser Anteil steigt anschließend bis einschließlich Position Th12 an (5,2 \% in Th1; 8,5\% in Th4; 10,6 \% in Th6; $11,6 \%$ in Th8; 14,2 \% in Th10) an, wobei in Position Th12 das Maximum (14,3\% in Th12) erreicht wird. Lumbal sinkt der relative Anteil der tiefen Atmung (10,9\% in L2; 9,3\% in L4). 
Der relative kardiale Spektralanteil nimmt im Frequenzbereich von 0,8 bis 1,8 $\mathrm{Hz}$ (Abb. 17b) von Position C3 (7,6\%) über C5 (7,5\%), Th1 (6,8\%) und Th4 (5,8\%) ab. In Position Th6 erfolgt eine Zunahme $(6,1 \%)$ des relativen Anteils. Kaudal von Th6 nimmt dieser kontinuierlich ab (5,7\% in Th8; 4,4 \% in Th10; 3,6 \% in Th12; 4,1\% in L2, 3,3 \% in L4).

Zusammenfassend betrachtet ist der Einfluss der Atmung auf die Flussdynamik des Liquors während der forcierten Atmung in allen Positionen vorhanden. Kaudal der Position Th4 dominiert der Einfluss der respiratorischen Frequenzkomponente auf den Fluss des Hirnwassers, insbesondere im unteren Bereich der Brustwirbelsäule (Th10 und Th12). Kranial des Bereichs Th4 nimmt der Einfluss der Herzaktivität auf die Flussdynamik des Hirnwassers auch in der tiefen Respirationsphase zu. Der Bereich Th4 tritt somit als Scheidepunkt hervor. 


\section{Diskussion}

\subsection{Fluss und Volumen des Hirnwassers entlang des Spinalkanals}

\subsubsection{Einordnung der Ergebnisse}

Mit der forcierten Inspiration fließt das Hirnwasser (s. Kapitel 3.1.4, Abb. 13) von der Lumbalregion entlang des gesamten Spinalkanals in Richtung Kopf in Übereinstimmung mit vorangegangenen Messungen im oberen Spinalkanal als auch mit Messungen an intrakraniellen Messpositionen (Klose et al. 2000; Friese et al. 2004; Yamada et al. 2013; Chen et al. 2015; Dreha-Kulaczewski et al. 2015; Dreha-Kulaczewski et al. 2017; Yildiz et al. 2017; Dreha-Kulaczewski et al. 2018; Spijkerman et al. 2018). Während der forcierten Exspiration verzeichnen oben genannte Arbeiten an kranialen Messpositionen sowie invasive Studien mittels Myelographie im gesamten Spinalkanal (Reitan 1941) einen Fluss nach kaudal. Dieser wurde in den vorliegenden Ergebnissen unterhalb Th4/Th6 beobachtet. Oberhalb Th1/Th4 wurde während der forcierten Exspiration ein fast vollständiges Erliegen des Flusses gemessen. Die sich hieraus ergebenden Netto-Flussvolumina sind kranial von Th4 kopfwärts (positiv) und kaudal von Th4 (negativ) lumbalwärts gerichtet. Daraus ergibt sich das Bild ähnlich einer Wasserscheide mit dem Scheitelpunkt auf Höhe des Herzens (Dreha-Kulaczewski et al. 2018).

Hervorzuheben ist, dass die Ergebnisse der Einzelmessungen und des Gruppenmittels trotz interindividueller Variabilität (s. Kapitel 3.1.1), die u. a. durch den zirkadianen Rhythmus der Liquorproduktion (Nilsson et al. 1992), durch die Atemleistung (Kaneko und Horie 2012) sowie durch weitere Aspekte wie Hydratationszustand und physikalische Leistungsfähigkeit (Dreha-Kulaczewski et al. 2017) beeinflusst werden, das gleiche Muster aufweisen. 


\subsubsection{Interpretation der Ergebnisse}

Nach Auffassung von Dreha-Kulaczewski et al. (Dreha-Kulaczewski et al. 2015; DrehaKulaczewski et al. 2017; Dreha-Kulaczewski et al. 2018) stellen Hirnwasser und venöses System ein eng miteinander kommunizierendes Flüssigkeitssystem dar. Die atmungsinduzierten Druckänderungen in der Brust- und Bauchhöhle übertragen sich über die Hohlvenen und das epidurale venöse System auf das Hirnwasser (Friese et al. 2004; Baron und Tunstall 2016). Weiterhin ist die Aufrechterhaltung eines konstanten intrakraniellen Volumens - Monro-Kellie-Doktrin (Weed 1929) - entscheidend für die Hirnwasserdynamik.

Während der Inspiration kommt es durch den Abfall des intrathorakalen Drucks auch zu einer Abnahme des Drucks in den intrathorakalen Venen - so auch in der oberen Hohlvene (Usubiaga et al. 1967; Brandes und Busse 2010) und im Venensystem der Halsregion (Dreha-Kulaczewski et al. 2017). Gleichzeitig nimmt der intraabdominelle Druck infolge des Tiefertretens des Zwerchfells zu und somit auch der Druck in den Abdominalvenen (Usubiaga et al. 1967; Brandes und Busse 2010). Während der Exspiration steigt der Druck in den intrathorakalen Venen und fält in den intraabdominellen Venen (Usubiaga et al. 1967; Grissmer 2012).

Zuflüsse der oberen und unteren Hohlvene werden durch externe (Plexus venosus vertebralis externus anterior et posterior, paravertebrale Lage) und interne (Plexus venosus vertebralis internus anterior et posterior, epidurale Lage) Venengeflechte verbunden (Batson 1967). Diese drainieren das venöse Blut der Wirbelsäule und des Rückenmarks in die kavalen und längs verlaufenden Venen des Azygossystems (Baron und Tunstall 2016). Die klappenlosen Venenplexus (Baron und Tunstall 2016) ermöglichen einen bidirektionalen Fluss (Batson 1942), sind untereinander intensiv vernetzt (Baron und Tunstall 2016) und sind aufgrund ihrer Lage im knöchernen Wirbelkanal auBerhalb der Körperhöhlen von intrathorakalen und intraabdominalen Druckänderungen geschützt (Henriques 1962). Die Venen der internen Venengeflechte besitzen zahlreiche Trabekel (Stringer et al. 2012). Diese verhindern eine Überdehnung und das Zusammensinken der Venen, regulieren die Richtung und Geschwindigkeit des venösen Blutflusses und sind in andere homöostatische Prozesse eingebunden (Stringer et al. 2012). 
Nach Usubiaga et al. verhält sich der Druck im Epiduralraum gleich dem Druck in den Hohlvenen (Usubiaga et al. 1967). Somit übertragen sich die intrathorakalen und intraabdominalen Druckänderungen (u. a. direkt über die Zwischenwirbellöcher [Foramina intervertebralia]) auf den Epiduralraum. Die direkte Übertragung ist möglich, da der Epiduralraum an der Grenze der thorakalen Höhle in engem Kontakt zur parietalen Pleura liegt (Macintosh und Mushin 1947), sodass der intrapleurale Druck über die paravertebral gelegenen externen Venengeflechte zum Epiduralraum mit den internen Venengeflechten geleitet wird (Shah 1981; Dreha-Kulaczewski et al. 2018). AnschlieBend wird der Druck über die Hirnhaut des Spinalkanals auf den Liquor weitergeleitet (Lockey et al. 1975; Williams 1981 b). Als zentrales Ergebnis stellten Usubiaga et al. gegensätzliche Druckschwankungen im Epiduralraum synchron zur Atmung heraus und schlussfolgerten, dass sich der spinale Epiduralraum in zwei funktionell zu unterscheidende Kompartimente aufteilt (Usubiaga et al. 1967). Diese Druckschwankungen werden auf die gleichzeitigen Druckänderungen in den entsprechenden Hohlvenen zurückgeführt. Im zerviko-thorakalen Kompartiment sinkt der Druck in der oberen Hohlvene und somit der epidurale Druck während der Inspiration und steigt mit der Exspiration. Die Drücke im lumbalen Kompartiment des Spinalkanals verhalten sich gegensätzlich dazu. Der epidurale Druck steigt mit der Inspiration und sinkt mit der Exspiration, entsprechend den Druckverhältnissen im Bereich der unteren Hohlvene. Interessanterweise, weist Usubiagas funktionelle Unterteilung des Epiduralraums Analogien zu der hier beobachteten Zweiteilung des Netto-Flussvolumens (s. Kapitel 3.1.4, Abb. 13b) auf. Parallele Studien des lumbalen epiduralen venösen Blutflusses sind eine Möglichkeit, beide Beobachtungen näher in Verbindung zu bringen (Dreha-Kulaczewski et al. 2018).

Zweite entscheidende Bedingung für die Dynamik des Hirnwassers ist, dass die Summe der Volumina von Gehirn, intrakraniellem Blut und Liquor im Schädel konstant bleibt. Dies wird auch als Monro-Kellie-Doktrin bezeichnet (Weed 1929). Durch den starren, nicht ausdehnbaren Schädel und die intrakraniellen, nicht komprimierbaren Strukturen führt ein Volumenanstieg einer der Komponenten entweder zu einem intrakraniellen Druckanstieg oder zu einer kompensatorischen Abnahme mindestens einer der anderen Komponenten (Marmarou und Beaumont 2004). Der Fluss des Hirnwassers nach kranial während der Inspiration folgt als Kompensation auf den verstärkten venösen Abfluss aus der Kopf-Hals-Region, wodurch das intrakranielle Volumen und folglich der intrakranielle Druck aufrechterhalten wird (Greitz et al. 1992; Klose et al. 2000; Yamada et al. 2013; Dreha-Kulaczewski et al. 2015; Dreha-Kulaczewski et al. 2017; Dreha-Kulaczewski et al. 2018). 
Dieser Kompensationsmechanismus wurde anschließend auf den Fluss des Hirnwassers im gesamten Spinalkanal übertragen und gefolgert, dass der venöse Abfluss aus der Kopf-Hals-Region während der forcierten Inspiration den entscheidenden Antrieb für den nach kranial gerichteten Liquorfluss entlang des gesamten Spinalkanals darstellt (Dreha-Kulaczewski et al. 2018).

Die hier beschriebenen Ergebnisse bezüglich der Inspiration an allen Positionen und der Exspiration unterhalb Th4/Th6 lassen sich in Einklang bringen mit den oben beschriebenen Zusammenhängen zwischen Atmung und Hirnwasserfluss. Aufschluss über den geringen, in der Richtung variablen, teilweise nach kranial gerichteten, Fluss des spinalen Liquorraums oberhalb Th4/Th6 während der Exspiration können weiterführende Messungen liefern, in denen gleichzeitig der epidurale Blutfluss und der Liquorfluss auf verschiedenen Höhen des Spinalkanals gemessen wird (Dreha-Kulaczewski et al. 2018).

\subsection{Kardialer versus respiratorischer Flussantrieb - unterschiedliche Dominanz in Abhängigkeit von der Lokalisation}

Die Ergebnisse zeigen, dass der Einfluss der Atmung auf den Fluss des Hirnwassers in allen Positionen entlang des Spinalkanals (s. Kapitel 3.2, Abb. 15, 16 und 17) vorhanden ist. Der Anteil der respiratorischen Frequenz relativ zur Summe aller Frequenzen nimmt von kranial nach kaudal zu. Der Anteil der kardialen Frequenzen nimmt hingegen von kranial nach kaudal ab (s. Kapitel 3.2, Abb. 15, 16 und 17). Damit stimmen die vorliegenden Ergebnisse mit denen von der Arbeitsgruppe um Friese überein (Friese et al. 2004). Sie quantifizierten den respiratorischen und kardialen Einfluss auf die Dynamik des Hirnwassers im Spinalkanal in weniger Positionen (C1, C2/C3, C6/C7, Th5, Th12) mittels echoplanarer Bildgebung.

Weitere Arbeiten zum Einfluss der Atmung und des Herzschlags auf die Dynamik des Hirnwassers beschränkten sich auf intrakranielle (Kao et al. 2008; Chen et al. 2015; Takizawa et al. 2017; Yildiz et al. 2017) oder ausschließlich zervikale (Daouk et al. 2017) Messpositionen. Daouk et al. quantifizierten das Verhältnis des kardialen und respiratorischen Einflusses in Position C2/C3 und stellten übereinstimmend mit den hier beschriebenen Werten (s. Kapitel 3.2, Abb. 17) fest, dass der kardiale Einfluss in dieser Position überwiegt. 
Die Beobachtung von Flusskomponenten mit höheren Frequenzen und niedrigeren Amplituden entlang des gesamten Spinalkanals, welche auf die pulsatile Herzaktion zurückzuführen sind, findet sich ebenso in früheren Arbeiten (Usubiaga et al. 1967; DrehaKulaczewski et al. 2015; Dreha-Kulaczewski et al. 2017; Dreha-Kulaczewski et al. 2018).

Im mittleren und unteren Bereich des Spinalkanals (kaudal Th4) dominiert die respiratorische im Vergleich zur kardialen Frequenzkomponente (s. Kapitel 3.2, Abb. 15, 16 und 17) - insbesondere im unteren Bereich der Brustwirbelsäule (Th10 und Th12). Die Ergebnisse sind übereinstimmend mit Arbeiten von Friese et al., nach denen der respiratorische Einfluss auf den Fluss des Liquors im Bereich Th12 seinen Höhepunkt erreicht (Friese et al. 2004).

Auffällig ist, dass der Ort des größten respiratorischen Einflusses mit der Projektion des Zwerchfells auf das Skelett zusammenfällt. Das Zwerchfell trennt die Brusthöhle von der Bauchhöhle (Anderhuber et al. 2012) und bewirkt während der Atmung große Druckänderungen in den o. g. Körperhöhlen und hat damit wahrscheinlich starken Einfluss auf den Flussantrieb des Hirnwassers.

Die Lage des Zwerchfells in Projektion auf das Skelett ist während der In- und Exspiration variabel. Während der Inspiration flacht das Zwerchfell ab und projiziert sich rechts dorsal auf Höhe Th11 und links auf Höhe Th11-12. Während der Exspiration projiziert sich das Zwerchfell rechts dorsal auf Höhe Th8 und links auf Höhe Th8-9 (Anderhuber et al. 2012).

Durch die forcierte Atmung werden Druckunterschiede zwischen Brust- und Bauchhöhle hervorgerufen. Die vorliegenden Flussmessungen legen einen Zusammenhang zwischen diesen Druckunterschieden und der unterschiedlichen Dominanz respiratorischer und kardialer Frequenzkomponenten auf den Antrieb des Liquorflusses in Abhängigkeit von der Position im Spinalkanal nahe. Für eine definitive Aussage sollten die Flussmessungen indes noch um Druckmessungen ergänzt werden. 


\subsection{Limitationen und Fehlerquellen}

\subsubsection{Fläche der ROI}

Die Fläche der ROI wurde in dem Gebiet mit dem stärksten Flusssignal definiert (s. Kapitel 2.6.1) und weicht demzufolge von der tatsächlichen transversalen Querschnittsfläche des spinalen Liquorraums ab. Diese Abweichung überträgt sich auf die ermittelten Werte von Geschwindigkeit und Fluss des Hirnwassers. Um die Fläche der ROI mit der tatsächlichen transversalen Querschnittsfläche des Spinalkanals zu korrelieren, könnten in weiteren Studien zusätzlich T2-gewichtete Aufnahmen erfolgen.

\subsubsection{Datenverwertung}

Von ursprünglich 190 verwertbaren Messdaten mussten 18 Bildserien ausgeschlossen werden (s. Kapitel 2.5 und 3). Somit reduziert sich die Anzahl an verwertbaren Messungen und wirkt sich auf die Standardabweichung vom Gruppenmittel aus (s. Tab. A1, A2, A4 und A5, Anhang). Eine kürzlich entwickelte modelbasierte Rekonstruktion (Tan et al. 2017) könnte in Bezug auf die Anzahl fehlgeschlagener Rekonstruktionen Abhilfe schaffen sowie zu einer verbesserten Bildqualität der Geschwindigkeitskarten führen. Diese stand zum Zeitpunkt der Auswertung noch nicht zur Verfügung.

\subsubsection{Messebene}

Idealerweise sollte die Messebene senkrecht zum Spinalkanal liegen, damit der Liquorfluss korrekt erfasst werden kann. Geringfügige Abweichungen der Messebene können beispielsweise durch Bewegung des Probanden im Scanner auftreten. Aufgrund der Anatomie des Spinalkanals betrifft dies allerdings nur Bewegungen schräg zum Spinalkanal, welche im Scanner wenig wahrscheinlich sind. Eine Korrektur etwaiger verbliebener Abweichungen von der Messebene bedürfte der Erfassung des Flusses in drei Dimensionen (Kollmeier et al. 2017). Zum gegenwärtigen Zeitpunkt steht diese Möglichkeit der Erfassung technisch noch nicht zur Verfügung. 


\subsubsection{Zeitliche Begrenzung der forcierten Atmung}

Die forcierte Atmung wurde, unabhängig vom individuellen Flusskurvenverlauf, zeitlich durch das Atemprotokoll begrenzt (s. Kapitel 2.6.1), auch wenn der Proband z. B. zu früh einatmete. Individuelle Abweichungen vom Atemprotokoll könnten im begrenzten Maße durch eine zeitliche Verschiebung der gesamten Flusskurve um einen fixen Versatz reduziert werden.

\subsubsection{Atemprotokoll}

Das Atemprotokoll lässt Freiraum bei der Ausführung der Atmung, insbesondere der Brust- und Bauchatmung, was die interindividuellen Schwankungen in der Atemleistung betont. Die Ergebnisse dieser Arbeit geben Anlass dazu, das Atemprotokoll in weiteren Studien bezüglich Brust- und Bauchatmung zu variieren und zu konkretisieren. Somit könnten u. a. interindividuelle Schwankungen methodisch reduziert werden. 


\section{Zusammenfassung}

In der vorliegenden Arbeit wurde erstmals systematisch und detailliert die Flussdynamik des Hirnwassers bei gesunden Probanden während der forcierten Atmung mittels nichtinvasiver flusssensitiver hochaufgelöster Phasenkontrast-Echtzeit-MRT entlang des gesamten Spinalkanals quantitativ untersucht.

Es fanden sich folgende Ergebnisse:

1. In welche Richtung fließt das Hirnwasser in den einzelnen Positionen entlang des Spinalkanals während der forcierten In- und Exspiration? Wieviel Hirnwasser fließt dabei? (s. Kapitel 3.1)

Mit der forcierten Inspiration fließt das Hirnwasser von der Lumbalregion entlang des gesamten Spinalkanals nach kranial. Während der begleitenden forcierten Exspiration erfolgt der Fluss des Hirnwassers unterhalb Th4/Th6 nach kaudal. Oberhalb des Bereichs Th4/Th6 ist der Fluss gering und in der Richtung variabel. Auch eine nach kranial gerichtete Flussbewegung wurde gemessen. Die sich hieraus ergebenden Netto-Flussvolumina sind oberhalb Th4 positiv und unterhalb Th4 negativ. Wie Abb. 13 (s. Kapitel 3.1.4) illustriert, ergibt sich der Eindruck einer Wasserscheide mit Scheitelpunkt in Herzhöhe.

Als Erklärung werden zwei voneinander abhängige und sich beeinflussende Flüssigkeitssysteme im Sinne eines Liquor-Venen-Systems angenommen. Atmungsinduzierte Druckänderungen in der Brust- und Bauchhöhle werden über die Hohlvenen und das epidurale venöse System auf das spinale Hirnwasser übertragen. Von entscheidender Bedeutung ist die kompensatorische Aufwärtsbewegung des Liquors während der forcierten Inspiration, um den verstärkten venösen Abfluss aus der Kopf-Hals-Region auszugleichen und das intrakranielle Volumen konstant zu halten. Dieser Kompensationsmechanismus bildet im gesamten Spinalkanal einen starken Antrieb für den nach kranial gerichteten Liquorfluss. 
2. Welchen Anteil haben jeweils Atmung und Herzaktion am Antrieb des Hirnwasserflusses entlang des Spinalkanals während der forcierten In- und Exspiration? (s. Kapitel 3.2)

Der Einfluss der Atmung auf den Fluss des Hirnwassers ist im Gruppenmittel entlang des gesamten Spinalkanals vorhanden und nimmt relativ zum Herzeinfluss von kranial nach kaudal zu, wohingegen der Anteil der kardialen Frequenzen von kranial nach kaudal abnimmt. Kaudal von Th4 dominiert der Einfluss der Atmung im Vergleich zum Einfluss des Herzens auf den Fluss des Hirnwassers.

Die vorliegenden Flussmessungen legen einen Zusammenhang zwischen den Druckunterschieden in der Brust- und Bauchhöhle und der unterschiedlichen Dominanz respiratorischer und kardialer Frequenzkomponenten auf den Antrieb des Liquorflusses in Abhängigkeit von der Position im Spinalkanal nahe. 


\section{Anhang}
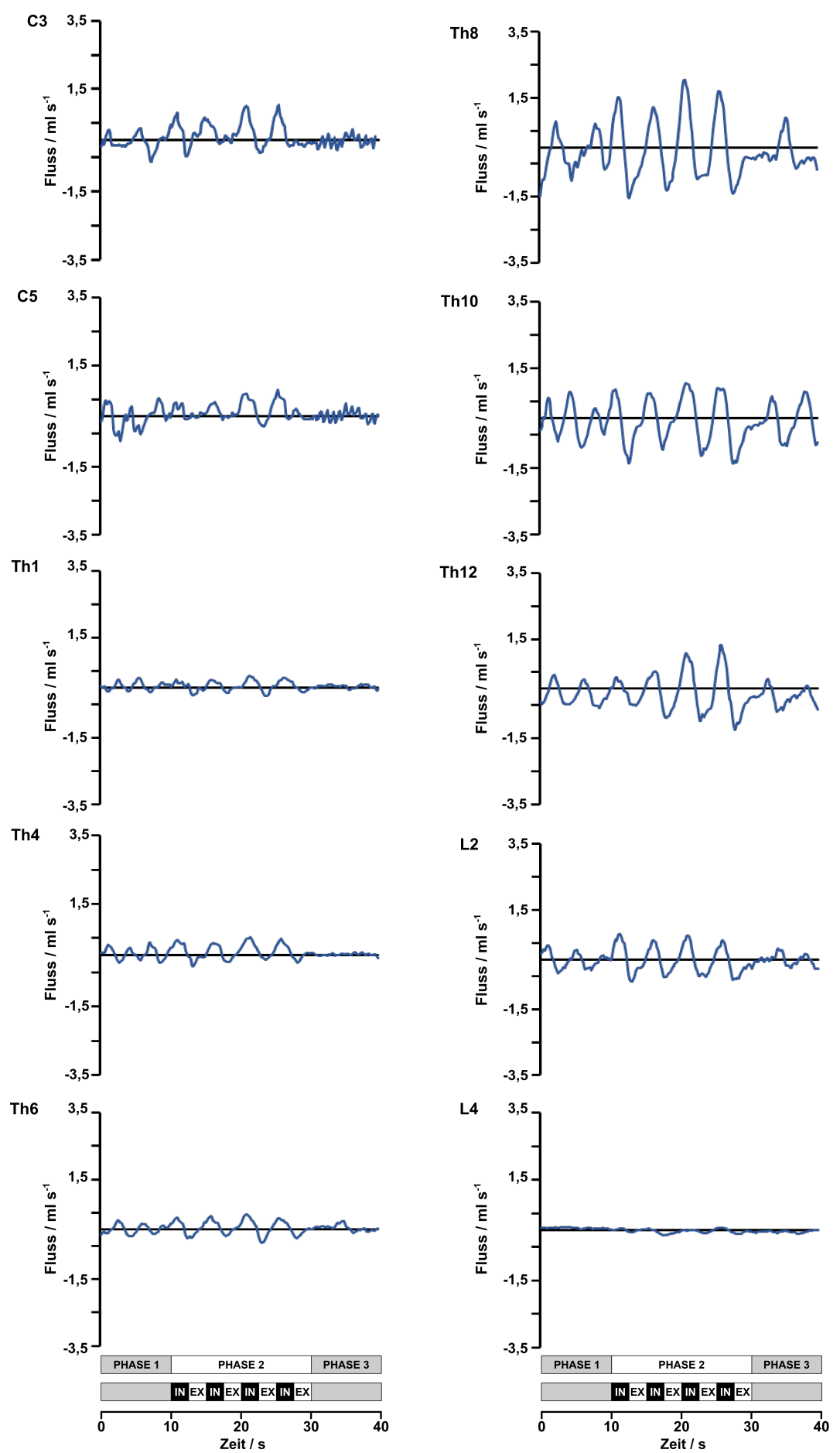

Abbildung A1: Flusskurven des Hirnwassers $\left(\mathrm{ml} \mathrm{s}^{-1}\right.$ ) entlang der Zeitserie (40 s) mit Atemprotokoll in allen Messpositionen mit angewandtem Tiefpassfilter des Probanden \#17 (Phase 1: normale Atmung, Phase 2: forcierte Atmung, Phase 3: normale Atmung, IN: forcierte Inspiration, EX: forcierte Exspiration); zu vergleichen mit Abb. 8 (s. Kapitel 3.1.1). 

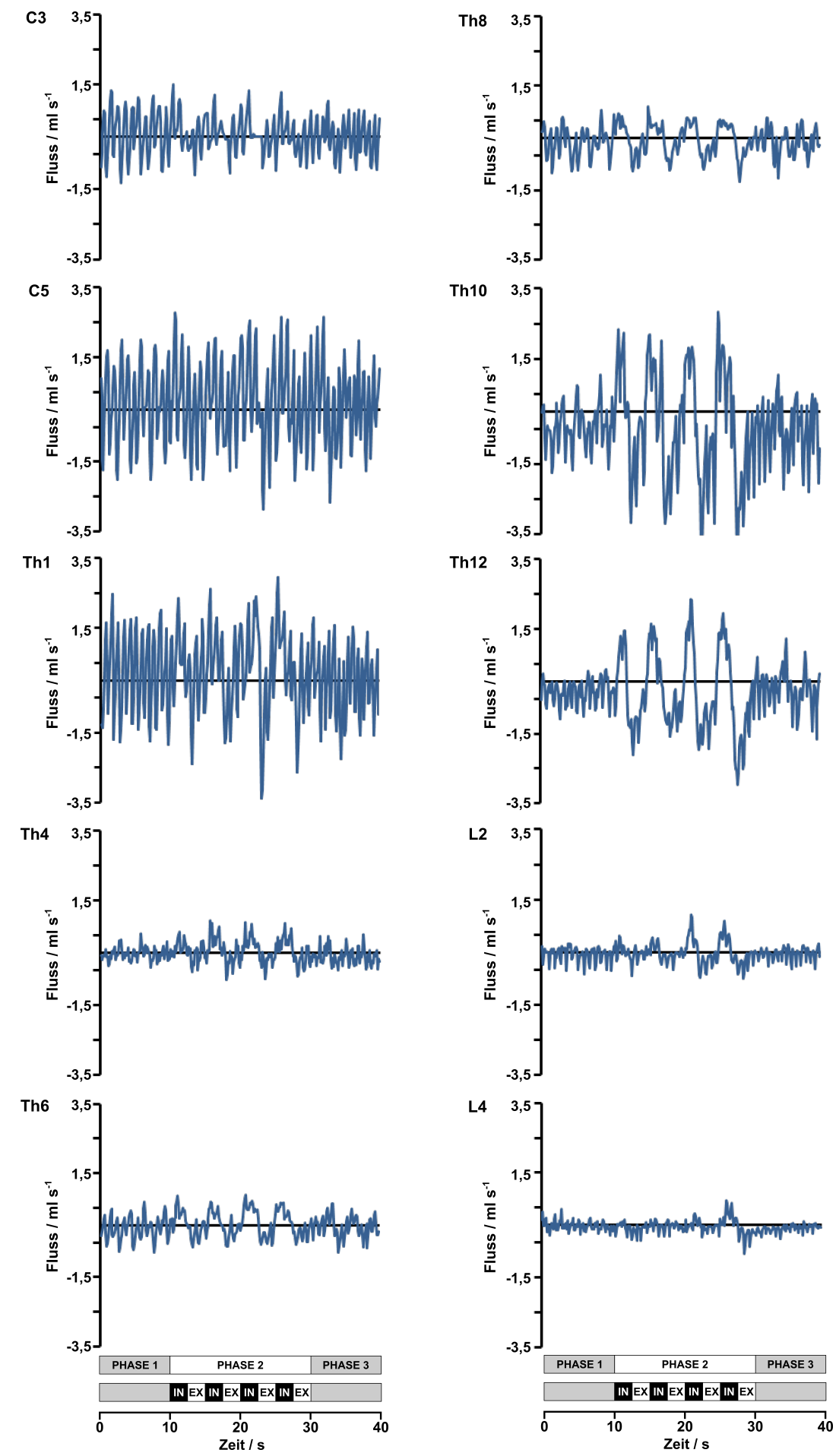

Abbildung A2: Flusskurven des Hirnwassers ( $\mathrm{ml} \mathrm{s}^{-1}$ ) entlang der Zeitserie (40 s) mit Atemprotokoll in allen Messpositionen des Probanden \#1 (Phase 1: normale Atmung, Phase 2: forcierte Atmung, Phase 3: normale Atmung, IN: forcierte Inspiration, EX: forcierte Exspiration); zu vergleichen mit Abb. 8 (s. Kapitel 3.1.1) und Abb. A3. 

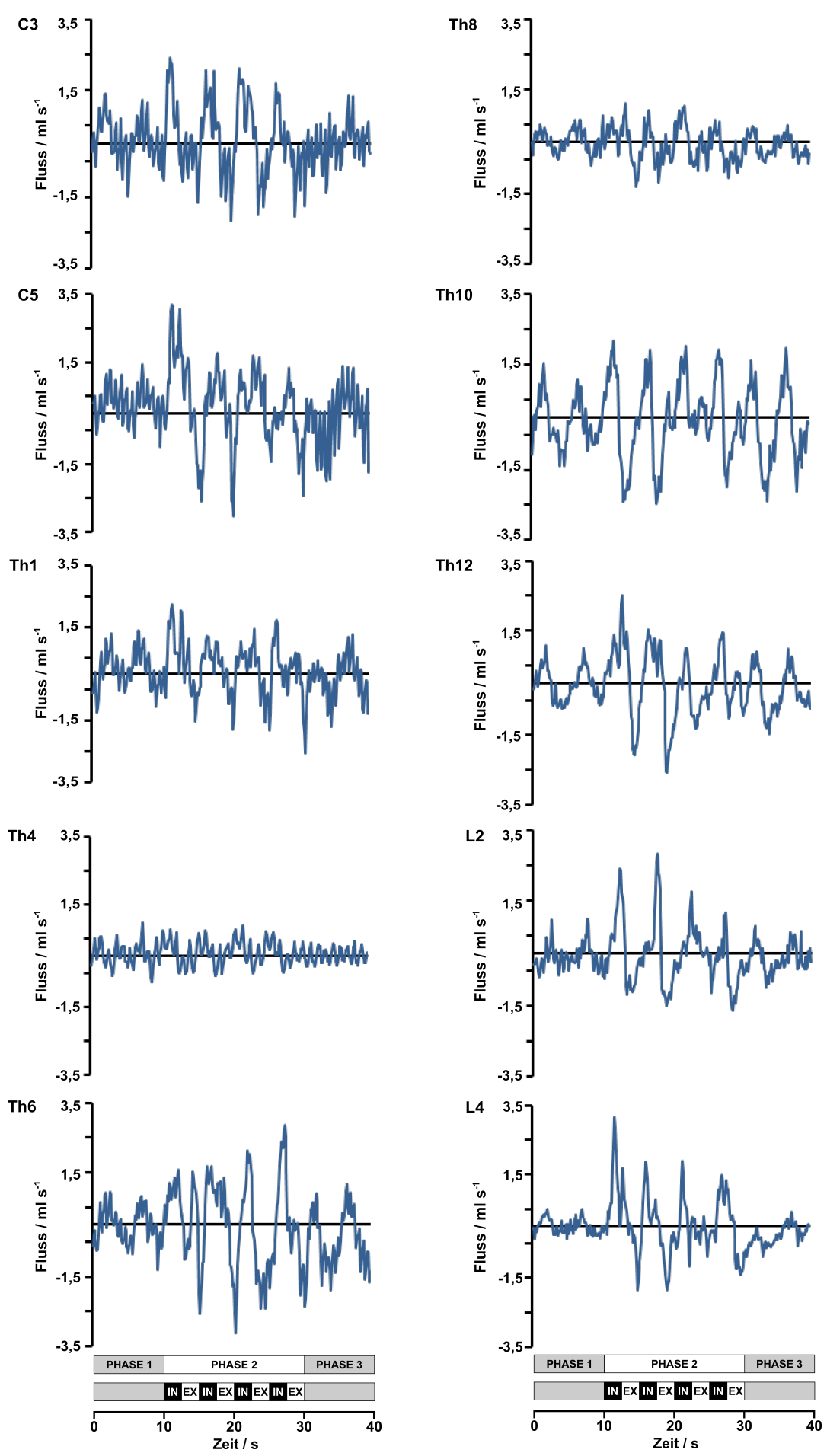

Abbildung A3: Flusskurven des Hirnwassers $\left(\mathrm{ml} \mathrm{s}^{-1}\right)$ entlang der Zeitserie (40 s) mit Atemprotokoll in allen Messpositionen des Probanden \#19 (Phase 1: normale Atmung, Phase 2: forcierte Atmung, Phase 3: normale Atmung, IN: forcierte Inspiration, EX: forcierte Exspiration); zu vergleichen mit Abb. 8 (s. Kapitel 3.1.1) und Abb. A2. 
Tabelle A1: Geschwindigkeitskodierung/VENC $\left(\mathrm{cm} \mathrm{s}^{-1}\right)$ aller Flussmessungen des Hirnwassers

\begin{tabular}{|c|c|c|c|c|c|c|c|c|c|c|}
\hline \multirow[t]{2}{*}{ Proband } & \multicolumn{10}{|c|}{ Messposition } \\
\hline & C3 & C5 & Th1 & Th4 & Th6 & Th8 & Th10 & Th12 & L2 & L4 \\
\hline$\# 1$ & 20 & 20 & 15 & 10 & 15 & 20 & 10 & 10 & 10 & 10 \\
\hline \#2 & 20 & 15 & 15 & 10 & 10 & 15 & 10 & 10 & 10 & n. a. \\
\hline \#3 & 20 & 20 & 10 & 20 & 10 & 10 & 10 & n. a. & 10 & 10 \\
\hline \#4 & 10 & 10 & 10 & 10 & 10 & 10 & 10 & 10 & 10 & 10 \\
\hline \#5 & 20 & 20 & 10 & 15 & 10 & 10 & 10 & n. a. & n. a. & 10 \\
\hline \#6 & 20 & 20 & 10 & 10 & 10 & 10 & 10 & 10 & 10 & 10 \\
\hline$\# 7$ & 20 & 20 & 10 & 15 & 15 & 10 & 10 & 10 & 10 & 10 \\
\hline \#8 & 20 & 20 & n. a. & 20 & 15 & 20 & 20 & 10 & 10 & 10 \\
\hline$\# 9$ & 20 & 15 & 15 & 15 & 15 & 10 & 10 & 10 & 10 & 10 \\
\hline$\# 10$ & 15 & n. a. & 15 & 15 & 10 & 10 & 20 & 10 & 10 & 10 \\
\hline$\# 11$ & 10 & 10 & 10 & 10 & 20 & 10 & 10 & n. a. & 10 & 10 \\
\hline \#12 & 10 & 15 & 10 & 10 & 10 & 10 & 10 & 10 & 10 & 10 \\
\hline \#13 & 15 & 20 & 10 & 10 & 10 & 10 & 10 & 10 & 10 & 10 \\
\hline$\# 14$ & n. a. & n. a. & 10 & 10 & 10 & 10 & 10 & 10 & n. a. & n. a. \\
\hline \#15 & 15 & 15 & 15 & 15 & 10 & 10 & 10 & n. a. & n. a. & 10 \\
\hline \#16 & 10 & 10 & 10 & 15 & 15 & 15 & 10 & n. a. & n. a. & 10 \\
\hline$\# 17$ & 20 & 20 & 20 & 20 & 20 & 20 & 10 & 10 & 10 & 10 \\
\hline$\# 18$ & n. a. & 10 & 10 & 10 & 10 & 10 & 10 & n. a. & 10 & n. a. \\
\hline \#19 & 20 & 20 & 10 & 20 & 20 & 20 & 20 & 20 & 20 & 10 \\
\hline
\end{tabular}

VENC (velocity encoding)

In Abhängigkeit von der maximal zu erwartenden Geschwindigkeit des Liquorflusses wurden die Flusskodierungsgradienten auf eine Geschwindigkeitskodierung von 10-40 $\mathrm{cm} \mathrm{s}^{-1}$ eingestellt. Bildserien, in denen Artefakte durch Anpassung der Messparameter nicht beseitigt werden konnten, ein Rekonstruktionsartefakt weiterhin bestand oder die Zielstruktur nicht sicher erkennbar war (nicht auswertbar, n. a.). 
Tabelle A2: Volumina des Hirnwassers (ml) jedes Probanden (\#1-19) entlang des Spinalkanals (C3-L4) während forcierter Atmung

\begin{tabular}{|c|c|c|c|c|c|c|c|c|c|c|c|c|c|c|c|c|c|c|c|c|c|}
\hline & & $\# 1$ & \#2 & $\# 3$ & \#4 & \#5 & \#6 & \#7 & \#8 & $\# 9$ & $\# 10$ & $\# 11$ & $\# 12$ & $\# 13$ & \#14 & $\# 15$ & $\# 16$ & $\# 17$ & $\# 18$ & $\# 19$ & Mittel \pm SD \\
\hline \multirow{3}{*}{ C3 } & IN & 0,6 & 0,4 & 0,4 & $-0,3$ & 1,1 & 0,1 & 1,0 & 0,3 & 0,2 & 0,6 & 0,2 & 0,3 & 0,6 & \multirow{3}{*}{ n. a. } & 0,3 & 0,9 & 1,3 & \multirow{3}{*}{ n. a. } & 1,4 & $0,6 \pm 0,4$ \\
\hline & EX & $-0,3$ & 0,0 & 0,1 & 0,8 & $-0,2$ & 0,0 & $-0,4$ & 0,1 & 0,1 & 0,4 & 0,2 & 0,5 & $-0,3$ & & 0,0 & 0,4 & $-0,2$ & & $-0,6$ & $0,0 \pm 0,4$ \\
\hline & NET & 0,3 & 0,4 & 0,5 & 0,6 & 1,0 & 0,1 & 0,6 & 0,4 & 0,3 & 0,9 & 0,4 & 0,8 & 0,4 & & 0,3 & 1,4 & 1,1 & & 0,8 & $0,6 \pm 0,3$ \\
\hline \multirow{3}{*}{ C5 } & $\mathrm{IN}$ & 1,4 & 0,6 & 0,3 & 0,2 & 1,2 & 0,3 & 0,5 & 0,0 & 0,6 & \multirow{3}{*}{ n. a. } & 0,1 & 0,3 & 0,2 & \multirow{3}{*}{ n. a. } & 0,1 & 1,0 & 0,8 & 0,8 & $-0,1$ & $0,5 \pm 0,4$ \\
\hline & EX & $-0,6$ & 0,2 & 0,0 & 0,6 & $-0,1$ & 0,5 & $-0,1$ & 0,0 & 0,3 & & 0,1 & 0,3 & $-0,1$ & & 0,2 & 0,4 & 0,2 & $-0,3$ & 1,5 & $0,2 \pm 0,5$ \\
\hline & NET & 0,9 & 0,7 & 0,2 & 0,8 & 1,0 & 0,7 & 0,5 & 0,0 & 1,0 & & 0,1 & 0,5 & 0,2 & & 0,4 & 1,4 & 0,8 & 0,4 & 1,4 & $0,7 \pm 0,4$ \\
\hline \multirow{3}{*}{ Th1 } & $\mathbb{I N}$ & 2,1 & 0,4 & 0,3 & 0,0 & 0,9 & 0,1 & 0,5 & \multirow{3}{*}{ n. a. } & 0,6 & 0,1 & 0,1 & 0,0 & 0,2 & 0,2 & 0,3 & 0,5 & 0,5 & 0,2 & 0,8 & $0,4 \pm 0,5$ \\
\hline & EX & $-0,5$ & $-0,1$ & 0,0 & 0,2 & $-0,3$ & 0,3 & 0,0 & & 0,3 & 0,0 & 0,2 & 0,2 & 0,1 & 0,0 & 0,4 & 0,1 & $-0,2$ & $-0,1$ & 0,5 & $0,1 \pm 0,2$ \\
\hline & NET & 1,6 & 0,3 & 0,3 & 0,3 & 0,6 & 0,4 & 0,5 & & 0,9 & 0,1 & 0,3 & 0,2 & 0,3 & 0,2 & 0,7 & 0,5 & 0,3 & 0,1 & 1,3 & $0,5 \pm 0,4$ \\
\hline \multirow{3}{*}{ Th4 } & IN & 0,6 & 0,1 & 0,1 & 0,1 & 0,6 & 0,1 & 1,0 & 0,1 & 0,1 & 1,2 & 0,2 & 0,0 & 0,2 & 0,2 & 0,7 & 0,7 & 0,8 & 0,0 & $-1,3$ & $0,3 \pm 0,5$ \\
\hline & EX & $-0,4$ & 0,0 & 0,0 & 0,0 & $-0,5$ & $-0,1$ & $-1,0$ & 0,0 & $-0,2$ & $-0,8$ & $-0,1$ & 0,0 & $-0,2$ & $-0,1$ & $-0,3$ & $-0,5$ & $-0,2$ & $-0,1$ & 1,6 & $-0,1 \pm 0,5$ \\
\hline & NET & 0,3 & 0,0 & 0,1 & 0,1 & 0,1 & 0,0 & $-0,1$ & 0,1 & $-0,1$ & 0,4 & 0,1 & 0,0 & 0,0 & 0,1 & 0,4 & 0,1 & 0,6 & $-0,1$ & 0,3 & $0,1 \pm 0,2$ \\
\hline \multirow{3}{*}{ Th6 } & IN & 0,8 & 0,3 & 0,1 & $-0,1$ & 0,5 & 0,1 & 0,2 & 0,1 & 0,2 & 0,4 & 0,2 & 0,1 & 0,4 & 0,3 & 0,2 & 0,4 & 0,6 & 0,0 & 0,3 & $0,3 \pm 0,2$ \\
\hline & EX & $-0,6$ & $-0,1$ & $-0,2$ & $-0,3$ & $-0,5$ & $-0,4$ & $-0,3$ & $-0,6$ & $-0,3$ & $-1,2$ & $-0,2$ & $-0,2$ & $-0,3$ & $-0,5$ & $-0,7$ & $-0,9$ & $-0,4$ & $-0,2$ & 0,1 & $-0,4 \pm 0,3$ \\
\hline & NET & 0,2 & 0,2 & $-0,1$ & $-0,4$ & 0,0 & $-0,3$ & $-0,1$ & $-0,4$ & $-0,1$ & $-0,8$ & 0,1 & $-0,1$ & 0,1 & $-0,2$ & $-0,5$ & $-0,5$ & 0,3 & $-0,1$ & 0,4 & $-0,1 \pm 0,3$ \\
\hline \multirow{3}{*}{ Th8 } & $\mathrm{IN}$ & 0,8 & 0,6 & 0,1 & $-0,2$ & 0,8 & 0,3 & 0,4 & 0,2 & 0,3 & $-0,4$ & 0,2 & 0,1 & 0,4 & 0,0 & 0,2 & 0,3 & 2,2 & $-0,1$ & 0,3 & $0,4 \pm 0,5$ \\
\hline & EX & $-1,0$ & $-0,7$ & $-0,3$ & $-0,3$ & $-2,1$ & $-0,5$ & $-2,2$ & $-1,5$ & $-0,7$ & $-1,5$ & $-0,4$ & $-0,3$ & $-0,5$ & $-0,5$ & $-0,7$ & $-1,2$ & $-2,3$ & $-0,1$ & $-0,8$ & $-0,9 \pm 0,7$ \\
\hline & NET & $-0,2$ & $-0,1$ & $-0,2$ & $-0,4$ & $-1,2$ & $-0,2$ & $-1,8$ & $-1,3$ & $-0,3$ & $-1,9$ & $-0,2$ & $-0,2$ & $-0,1$ & $-0,4$ & $-0,6$ & $-0,9$ & 0,0 & $-0,2$ & $-0,5$ & $-0,6 \pm 0,6$ \\
\hline \multirow{3}{*}{ Th10 } & $\mathrm{IN}$ & 2,2 & 0,9 & 0,2 & 0,4 & 1,8 & 1,6 & 1,1 & 2,8 & 0,4 & 2,3 & 0,6 & 0,2 & 0,7 & 0,1 & 0,8 & 0,8 & 1,3 & 0,1 & 1,9 & $1,1 \pm 0,8$ \\
\hline & EX & $-4,2$ & $-0,7$ & $-1,0$ & $-0,9$ & $-2,6$ & $-2,6$ & $-1,3$ & $-3,9$ & $-0,9$ & $-2,7$ & $-0,8$ & $-1,4$ & $-1,5$ & $-0,2$ & $-1,0$ & $-1,8$ & $-1,8$ & $-0,9$ & $-1,8$ & $-1,7 \pm 1,1$ \\
\hline & NET & $-2,0$ & 0,3 & $-0,8$ & $-0,5$ & $-0,9$ & $-1,0$ & $-0,2$ & $-1,1$ & $-0,4$ & $-0,4$ & $-0,2$ & $-1,2$ & $-0,8$ & $-0,1$ & $-0,2$ & $-1,1$ & $-0,5$ & $-0,8$ & 0,1 & $-0,6 \pm 0,5$ \\
\hline \multirow{3}{*}{ Th12 } & $\mathrm{IN}$ & 1,7 & 0,6 & & 0,1 & & 1,4 & 0,4 & 3,0 & 0,5 & 0,8 & & 0,4 & 0,3 & 0,1 & & & 1,2 & & 0,6 & $0,6 \pm 0,8$ \\
\hline & EX & $-3,1$ & $-0,9$ & n. a. & $-0,8$ & n. a. & $-1,4$ & $-0,6$ & $-3,7$ & $-1,1$ & $-2,2$ & n. a. & $-0,5$ & $-0,2$ & $-0,5$ & n. a. & n. a. & $-1,7$ & n. a. & $-0,7$ & $-1,3 \pm 1,1$ \\
\hline & NET & $-1,4$ & $-0,3$ & & $-0,7$ & & 0,1 & $-0,1$ & $-0,6$ & $-0,6$ & $-1,4$ & & $-0,1$ & 0,1 & $-0,3$ & & & $-0,5$ & & $-0,1$ & $-0,5 \pm 0,5$ \\
\hline \multirow{3}{*}{ L2 } & $\mathrm{IN}$ & 0,4 & 0,2 & $-0,1$ & 0,5 & & 1,2 & 0,3 & 2,0 & 0,3 & 0,8 & 0,1 & 0,0 & $-0,1$ & \multirow{3}{*}{ n. a. } & \multirow{3}{*}{ n. a. } & \multirow{3}{*}{ n. a. } & 0,9 & $-0,2$ & 0,2 & $0,4 \pm 0,6$ \\
\hline & EX & $-0,5$ & $-0,3$ & 0,0 & $-0,1$ & n. a. & $-1,0$ & $-0,5$ & $-1,4$ & $-0,4$ & $-1,2$ & $-0,3$ & 0,1 & $-0,1$ & & & & $-1,0$ & $-0,2$ & $-0,3$ & $-0,5 \pm 0,5$ \\
\hline & NET & $-0,1$ & $-0,1$ & $-0,1$ & 0,4 & & 0,2 & $-0,2$ & 0,6 & $-0,1$ & $-0,4$ & $-0,2$ & 0,1 & $-0,2$ & & & & $-0,1$ & $-0,4$ & 0,0 & $0,0 \pm 0,3$ \\
\hline \multirow{3}{*}{ L4 } & $\mathrm{IN}$ & 0,1 & & 0,0 & 0,4 & 0,1 & 0,4 & 0,1 & 0,9 & 0,2 & 0,2 & 0,2 & 0,1 & 0,0 & \multirow{3}{*}{ n. a. } & 0,4 & 0,4 & 0,0 & \multirow{3}{*}{ n. a. } & 0,8 & $0,3 \pm 0,3$ \\
\hline & EX & $-0,3$ & n. a. & 0,0 & $-0,2$ & 0,0 & $-0,4$ & $-0,1$ & $-1,0$ & $-0,2$ & $-0,3$ & $-0,1$ & 0,1 & 0,0 & & 0,0 & 0,2 & $-0,2$ & & $-0,3$ & $-0,2 \pm 0,3$ \\
\hline & NET & $-0,2$ & & 0,0 & 0,2 & 0,1 & 0,0 & 0,0 & $-0,2$ & 0,0 & $-0,1$ & 0,1 & 0,2 & 0,0 & & 0,3 & 0,5 & $-0,2$ & & 0,6 & $0,1 \pm 0,2$ \\
\hline
\end{tabular}

Individuelle und über alle Probanden gemittelte (Mittel) in- und exspiratorische Volumina (IN, EX) des Hirnwassers pro Messposition bezogen auf 2,5 Sekunden und die jeweilige Standardabweichung (SD). Sowie das individuelle und über alle Probanden gemittelte (Mittel) Netto-Volumen (NET = IN + EX) des Hirnwassers pro Messposition, welches während der Ein- und Ausatmung bezogen auf einen Atemzyklus (5 Sekunden) fließt mit entsprechender Standardabweichung (SD). Bildserien, in denen Artefakte durch Anpassung der Messparameter nicht beseitigt werden konnten, ein Rekonstruktionsartefakt weiterhin bestand oder die Zielstruktur nicht sicher erkennbar war (nicht auswertbar, n. a.). 
Tabelle A3: Signifikante Unterschiede ( $p$-Wert $\leq 0,05)$ zwischen in- und exspiratorischen Volumina sowie Netto-Volumina $(\mathrm{ml})$ des Hirnwassers im Gruppenmittel aller Messpositionen während forcierter Atmung

\begin{tabular}{|c|c|c|c|}
\hline \multirow[t]{2}{*}{ Position } & \multicolumn{3}{|c|}{ Positionen, zu denen signifikante Unterschiede bestehen während } \\
\hline & Inspiration & Exspiration & Netto \\
\hline $\mathrm{C} 3$ & & Th8, Th10, Th12 & $\begin{array}{l}\text { Th4, Th6, Th8, Th10, Th12 } \\
\text { L2, L4 }\end{array}$ \\
\hline C5 & & Th8, Th10, Th12 & $\begin{array}{l}\text { Th4, Th6, Th8, Th10, Th12 } \\
\text { L2, L4 }\end{array}$ \\
\hline Th1 & Th10 & Th8, Th10, Th12 & Th6, Th8, Th10, Th12, L2 \\
\hline Th4 & Th10 & Th8, Th10, Th12 & C3, C5, Th8, Th10, Th12 \\
\hline Th6 & Th10 & Th10, Th12 & C3, C5, Th1, Th8, Th10 \\
\hline Th8 & Th10 & $\begin{array}{l}\text { C3, C5, Th1, Th4, } \\
\text { Th10, L4 }\end{array}$ & $\begin{array}{l}\text { C3, C5, Th1, Th4, Th6, L2 } \\
\text { L4 }\end{array}$ \\
\hline Th10 & $\begin{array}{l}\text { Th1, Th4, Th6, } \\
\text { Th8, L4 }\end{array}$ & $\begin{array}{l}\text { C3, C5, Th1, Th4, } \\
\text { Th6, Th8, L2, L4 }\end{array}$ & $\begin{array}{l}\text { C3, C5, Th1, Th4, Th6, L2, } \\
\text { L4 }\end{array}$ \\
\hline Th12 & & $\begin{array}{l}\text { C3, C5, Th1, Th4, } \\
\text { Th6, L2, L4 }\end{array}$ & C3, C5, Th1, Th4, L4 \\
\hline L2 & & Th10, Th12 & C3, C5, Th1, Th8, Th10 \\
\hline L4 & Th10 & Th8, Th10, Th12 & C3, C5, Th8, Th10, Th12 \\
\hline
\end{tabular}

Die zweite bis vierte Spalte gibt jeweils an, in welchen Positionen signifikante Unterschiede während der In- und Exspiration sowie Netto zu der Position in der ersten Spalte bestehen. 
Tabelle A4: Relativer Spektralanteil (\%) bei 0,2 Hz während forcierter Atmung

\begin{tabular}{|c|c|c|c|c|c|c|c|c|c|c|}
\hline \multirow[t]{2}{*}{ Proband } & \multicolumn{10}{|c|}{ Messposition } \\
\hline & C3 & C5 & Th1 & Th4 & Th6 & Th8 & Th10 & Th12 & L2 & L4 \\
\hline$\# 1$ & 7,6 & 6,5 & 8,5 & 13,5 & 18,8 & 22,1 & 16,5 & 19,9 & 12,4 & 7,2 \\
\hline \#2 & 2,8 & 2,8 & 12,0 & 8,3 & 6,3 & 12,9 & 13,5 & 14,0 & 10,4 & n. a. \\
\hline \#3 & 2,4 & 2,5 & 4,0 & 3,3 & 6,1 & 7,0 & 7,0 & n. a. & 2,2 & 2,5 \\
\hline$\# 4$ & 5,6 & 2,7 & 3,2 & 1,5 & 7,3 & 2,6 & 10,5 & 7,7 & 13,5 & 10,8 \\
\hline \#5 & 6,0 & 7,3 & 6,7 & 16,6 & 18,1 & 16,8 & 20,2 & n. a. & n. a. & 5,5 \\
\hline \#6 & 3,1 & 1,0 & 3,0 & 5,5 & 8,0 & 12,1 & 15,9 & 17,2 & 17,9 & 17,6 \\
\hline$\# 7$ & 7,0 & 4,6 & 5,1 & 14,1 & 15,8 & 14,7 & 21,8 & 14,3 & 13,9 & 12,4 \\
\hline \#8 & 0,8 & 2,9 & n. a. & 1,5 & 4,5 & 5,8 & 13,5 & 17,2 & 19,0 & 16,6 \\
\hline$\# 9$ & 1,3 & 2,3 & 2,4 & 3,5 & 6,2 & 8,6 & 10,2 & 13,1 & 11,3 & 12,0 \\
\hline$\# 10$ & 1,3 & n. a. & 3,1 & 8,3 & 8,4 & 9,4 & 16,0 & 13,4 & 9,7 & 9,5 \\
\hline$\# 11$ & 0,6 & 0,9 & 0,5 & 7,5 & 9,9 & 11,7 & 20,4 & n. a. & 10,7 & 11,2 \\
\hline \#12 & 2,2 & 2,5 & 5,2 & 4,0 & 5,9 & 6,9 & 11,4 & 9,9 & 5,2 & 2,1 \\
\hline \#13 & 6,7 & 5,4 & 2,2 & 9,1 & 15,0 & 19,9 & 12,9 & 14,6 & 4,3 & 3,5 \\
\hline$\# 14$ & n. a. & n. a. & 5,0 & 10,0 & 13,9 & 10,1 & 8,1 & 13,5 & n. a. & n. a. \\
\hline \#15 & 3,4 & 2,9 & 5,6 & 6,4 & 7,9 & 7,7 & 12,6 & n. a. & n. a. & 5,4 \\
\hline \#16 & 2,3 & 3,8 & 3,0 & 14,4 & 14,7 & 18,3 & 17,5 & n. a. & n. a. & 8,9 \\
\hline$\# 17$ & 7,1 & 4,9 & 8,2 & 12,8 & 14,4 & 19,7 & 15,1 & 15,6 & 15,9 & 9,3 \\
\hline$\# 18$ & n. a. & 5,5 & 4,6 & 5,3 & 5,9 & 5,1 & 9,9 & n. a. & 2,2 & n. a. \\
\hline$\# 19$ & 14,7 & 11,9 & 10,6 & 16,6 & 14,1 & 9,1 & 17,3 & 16,1 & 14,4 & 14,5 \\
\hline Mittel & 4,4 & 4,1 & 5,2 & 8,5 & 10,6 & 11,6 & 14,2 & 14,3 & 10,9 & 9,3 \\
\hline$\pm \mathrm{SD}$ & $\pm 3,5$ & $\pm 2,6$ & $\pm 2,9$ & $\pm 4,8$ & $\pm 4,5$ & $\pm 5,5$ & $\pm 4,1$ & $\pm 3,0$ & $\pm 5,2$ & $\pm 4,6$ \\
\hline
\end{tabular}

Anteil der respiratorischen Frequenz von $0,2 \mathrm{~Hz}$ relativ zur Summe aller Frequenzen in den entsprechenden Messpositionen aller Probanden sowie die Mittelwerte (Mittel) und Standardabweichungen (SD). Bildserien, in denen Artefakte durch Anpassung der Messparameter nicht beseitigt werden konnten, ein Rekonstruktionsartefakt weiterhin bestand oder die Zielstruktur nicht sicher erkennbar war (nicht auswertbar, n. a.). 
Tabelle A5: Relativer Spektralanteil (\%) bei 0,8 bis 1,8 Hz während forcierter Atmung

\begin{tabular}{|c|c|c|c|c|c|c|c|c|c|c|}
\hline \multirow[t]{2}{*}{ Proband } & \multicolumn{10}{|c|}{ Messposition } \\
\hline & C3 & C5 & Th1 & Th4 & Th6 & Th8 & Th10 & Th12 & L2 & L4 \\
\hline$\# 1$ & 7,0 & 8,8 & 5,8 & 5,0 & 5,4 & 4,5 & 3,6 & 2,7 & 4,7 & 6,0 \\
\hline \#2 & 6,6 & 6,2 & 7,6 & 5,1 & 4,9 & 9,8 & 6,2 & 7,4 & 5,5 & n. a. \\
\hline \#3 & 10,8 & 4,5 & 10,5 & 8,2 & 9,0 & 10,0 & 3,8 & n. a. & 3,3 & 4,5 \\
\hline$\# 4$ & 5,3 & 5,4 & 4,8 & 4,3 & 5,1 & 4,3 & 8,2 & 6,7 & 4,9 & 2,5 \\
\hline \#5 & 6,5 & 7,0 & 5,6 & 5,8 & 4,0 & 4,8 & 2,8 & n. a. & n. a. & 4,8 \\
\hline \#6 & 7,8 & 8,5 & 7,1 & 4,6 & 7,5 & 3,6 & 4,8 & 2,6 & 2,9 & 2,9 \\
\hline$\# 7$ & 11,3 & 7,1 & 10,9 & 9,7 & 8,9 & 6,9 & 3,0 & 3,0 & 2,6 & 1,5 \\
\hline \#8 & 3,5 & 3,4 & n. a. & 3,8 & 4,1 & 3,1 & 4,2 & 2,3 & 3,0 & 3,1 \\
\hline$\# 9$ & 11,3 & 10,3 & 5,5 & 6,1 & 9,4 & 9,5 & 5,1 & 4,1 & 4,1 & 2,3 \\
\hline$\# 10$ & 3,7 & n. a. & 3,0 & 3,1 & 4,7 & 3,9 & 2,3 & 2,9 & 2,7 & 3,7 \\
\hline$\# 11$ & 5,7 & 5,4 & 5,8 & 4,8 & 7,1 & 6,6 & 4,6 & n. a. & 6,0 & 4,1 \\
\hline \#12 & 6,9 & 5,1 & 4,5 & 4,5 & 9,0 & 5,3 & 3,3 & 2,2 & 3,4 & 2,8 \\
\hline \#13 & 5,8 & 9,4 & 6,6 & 4,4 & 3,6 & 2,6 & 1,9 & 2,6 & 5,5 & 2,9 \\
\hline$\# 14$ & n. a. & n. a. & 5,1 & 4,1 & 3,7 & 3,1 & 3,4 & 2,6 & n. a. & n. a. \\
\hline \#15 & 11,3 & 7,9 & 7,8 & 9,2 & 8,0 & 8,1 & 6,0 & n. a. & n. a. & 3,1 \\
\hline \#16 & 10,2 & 7,0 & 9,4 & 7,0 & 5,9 & 6,6 & 5,5 & n. a. & n. a. & 3,0 \\
\hline$\# 17$ & 11,4 & 12,7 & 9,8 & 9,1 & 7,4 & 6,7 & 6,0 & 4,4 & 4,2 & 3,7 \\
\hline$\# 18$ & n. a. & 16,9 & 9,5 & 8,2 & 6,6 & 6,5 & 6,1 & n. a. & 4,6 & n. a. \\
\hline$\# 19$ & 4,6 & 2,8 & 3,5 & 2,8 & 2,4 & 2,9 & 2,7 & 2,8 & 3,7 & 2,3 \\
\hline Mittel & 7,6 & 7,5 & 6,8 & 5,8 & 6,1 & 5,7 & 4,4 & 3,6 & 4,1 & 3,3 \\
\hline$\pm \mathrm{SD}$ & $\pm 2,8$ & $\pm 3,4$ & $\pm 2,3$ & $\pm 2,1$ & $\pm 2,1$ & $\pm 2,3$ & $\pm 1,6$ & $\pm 1,6$ & $\pm 1,1$ & $\pm 1,1$ \\
\hline
\end{tabular}

Anteil der kardialen Frequenzen (Maximum im Bereich von 0,8-1,8 Hz) relativ zur Summe aller Frequenzen in den entsprechenden Messpositionen aller Probanden sowie die Mittelwerte (Mittel) und Standardabweichungen (SD). Bildserien, in denen Artefakte durch Anpassung der Messparameter nicht beseitigt werden konnten, ein Rekonstruktionsartefakt weiterhin bestand oder die Zielstruktur nicht sicher erkennbar war (nicht auswertbar, n. a.). 


\section{Literaturverzeichnis}

Anderhuber F, Timm JF, Pera F, Peuker ET: Innere Organe in Thorax, Abdomen und Becken. In: Anderhuber F, Pera F, Streicher J (Hrsg.): Waldeyer - Anatomie des Menschen: Lehrbuch und Atlas in einem Band. 19. Auflage; de Gruyter, Berlin Boston 2012, 427-695

Askar FZ, Kocabas S, Yucel S, Samancilar O, Cetin H, Uyar M (2007): The efficacy of intrathecal morphine in post-thoracotomy pain management. J Int Med Res $\underline{35}$, 314-322

Baron EM, Tunstall R: Back. In: Standring S (Hrsg.): Gray's Anatomy: The Anatomical Basis of Clinical Practice. 41. Auflage; Elsevier, Philadelphia 2016, 710-750

Batson OV (1942): The vertebral vein system as a mechanism for the spread of metastases. Am J Roentgenol Radium Ther $\underline{48}, 715-718$

Batson OV (1967): The vertebral system of veins as a means for cancer dissemination. Prog Clin Cancer $\underline{3}, 1-18$

Battal B, Kocaoglu M, Bulakbasi N, Husmen G, Tuba Sanal H, Tayfun C (2011): Cerebrospinal fluid flow imaging by using phase-contrast MR technique. $\mathrm{Br} \mathrm{J}$ Radiol 84 , 758-765

Bechmann I, Nitsch R, Pera F, Winkelmann A, Stahnisch F: Zentrales Nervensystem, Systema nervosum centrale, Gehirn, Encephalon und Rückenmark, Medulla spinalis. In: Anderhuber F, Pera F, Streicher J (Hrsg.): Waldeyer - Anatomie des Menschen: Lehrbuch und Atlas in einem Band. 19. Auflage; de Gruyter, Berlin Boston 2012, 945-1126

Bering EA (1955): Choroid plexus and arterial pulsation of cerebrospinal fluid; demonstration of the choroid plexuses as a cerebrospinal fluid pump. AMA Arch Neurol Psychiatry $\underline{73}, 165-172$

Brandes R, Busse R: Kreislauf. In: Schmidt RF, Lang F, Heckmann M (Hrsg.): Physiologie des Menschen mit Pathophysiologie. 31. Auflage; Springer Medizin Verlag, Heidelberg 2010, 573-626 
Brown PD, Davies SL, Speake T, Millar ID (2004): Molecular mechanisms of cerebrospinal fluid production. Neuroscience 129, 957-970

Bryant DJ, Payne JA, Firmin DN, Longmore DB (1984): Measurement of flow with NMR imaging using a gradient pulse and phase difference technique. J Comput Assist Tomogr $\underline{8}, 588-593$

Chen L, Beckett A, Verma A, Feinberg DA (2015): Dynamics of respiratory and cardiac CSF motion revealed with real-time simultaneous multi-slice EPI velocity phase contrast imaging. Neuroimage 122, 281-287

Chitiboi T, Hennemuth A, Tautz L, Huellebrand M, Frahm J, Linsen L, Hahn H: Contextbased segmentation and analysis of multi-cycle real-time cardiac MRI. In: 2014 IEEE 11th International Symposium on Biomedical Imaging (ISBI). 2. Auflage; o.V., Peking 2014, 943-946

Damkier HH, Brown PD, Praetorius J (2013): Cerebrospinal fluid secretion by the choroid plexus. Physiol Rev 93, 1847-1892

Daouk J, Bouzerar R, Baledent O (2017): Heart rate and respiration influence on macroscopic blood and CSF flows. Acta Radiol 58, 977-982

Dreha-Kulaczewski S, Joseph AA, Merboldt K-D, Ludwig H-C, Gärtner J, Frahm J (2015): Inspiration is the major regulator of human CSF flow. J Neurosci 35, 2485-2491

Dreha-Kulaczewski S, Joseph AA, Merboldt K-D, Ludwig H-C, Gärtner J, Frahm J (2017): Identification of the Upward Movement of Human CSF In Vivo and its Relation to the Brain Venous System. J Neurosci 37, 2395-2402

Dreha-Kulaczewski S, Konopka M, Joseph AA, Kollmeier J, Merboldt K-D, Ludwig H-C, Gärtner J, Frahm J (2018): Respiration and the watershed of spinal CSF flow in humans. Sci Rep 8, 5594

Du Boulay GH (1966): Pulsatile movements in the CSF pathways. Br J Radiol 39, 255262

Du Boulay G, O'Connell J, Currie J, Bostick T, Verity P (1972): Further investigations on pulsatile movements in the cerebrospinal fluid pathways. Acta Radiol Diagn (Stockh) $13,496-523$ 
Friese S, Hamhaber U, Erb M, Kueker W, Klose U (2004): The influence of pulse and respiration on spinal cerebrospinal fluid pulsation. Invest Radiol 39, 120-130

Galarza M, Giménez Á, Valero J, Pellicer OP, Amigó JM (2014): Computational fluid dynamics of ventricular catheters used for the treatment of hydrocephalus: a 3D analysis. Childs Nerv Syst $\underline{30}, 105-116$

Greitz D, Wirestam R, Franck A, Nordell B, Thomsen C, Ståhlberg F (1992): Pulsatile brain movement and associated hydrodynamics studied by magnetic resonance phase imaging. Neuroradiology 34, 370-380

Greitz D, Franck A, Nordell B (1993): On the pulsatile nature of intracranial and spinal CSF-circulation demonstrated by MR imaging. Acta Radiol 34, 321-328

Grissmer S: Blutkreislauf. In: Behrends JC, Bischofberger J, Deutzmann R, Ehmke H, Frings S, Grissmer S, Hoth M, Kurtz A, Leipziger J, Müller F et al. (Hrsg.): Physiologie. 2. Auflage; Thieme, Stuttgart 2012, 111-162

Henriques CQ (1962): The veins of the vertebral column and their role in the spread of cancer. Ann R Coll Surg Engl 31, 1-22

Illes S (2017): More than a drainage fluid: the role of CSF in signaling in the brain and other effects on brain tissue. Handb Clin Neurol 146, 33-46

Jessen NA, Munk ASF, Lundgaard I, Nedergaard M (2015): The Glymphatic System: A Beginner's Guide. Neurochem Res 40, 2583-2599

Johanson CE, Duncan JA, Klinge PM, Brinker T, Stopa EG, Silverberg GD (2008): Multiplicity of cerebrospinal fluid functions: New challenges in health and disease. Cerebrospinal Fluid Res $\underline{5}, 10$

Joseph AA, Merboldt K-D, Voit D, Zhang S, Uecker M, Lotz J, Frahm J (2012): Real-time phase-contrast MRI of cardiovascular blood flow using undersampled radial fast low-angle shot and nonlinear inverse reconstruction. NMR Biomed 25, 917-924

Joseph A, Kowallick JT, Merboldt K-D, Voit D, Schaetz S, Zhang S, Sohns JM, Lotz J, Frahm J (2014): Real-time flow MRI of the aorta at a resolution of $40 \mathrm{msec}$. J Magn Reson Imaging 40, 206-213 
Kaneko $\mathrm{H}$, Horie $\mathrm{J}$ (2012): Breathing movements of the chest and abdominal wall in healthy subjects. Respir Care 57, 1442-1451

Kao Y-H, Guo W-Y, Liou AJ-K, Hsiao Y-H, Chou C-C (2008): The respiratory modulation of intracranial cerebrospinal fluid pulsation observed on dynamic echo planar images. Magn Reson Imaging 26, 198-205

Klose U, Strik C, Kiefer C, Grodd W (2000): Detection of a relation between respiration and CSF pulsation with an echoplanar technique. J Magn Reson Imaging 11, 438444

Koh L, Zakharov A, Johnston M (2005): Integration of the subarachnoid space and lymphatics: is it time to embrace a new concept of cerebrospinal fluid absorption? Cerebrospinal Fluid Res 2, 6

Kollmeier JM, Joseph AA, Voit D, Tan Z, Merboldt K-D, Frahm J: 2D In-plane Flow MRI in Real Time. ESMRMB Annual Scientific Meeting, Barcelona, 19.-21. Oktober 2017 (Poster)

Lockey P, Poots G, Williams B (1975): Theoretical aspects of the attenuation of pressure pulses within cerebrospinal-fluid pathways. Med Biol Eng 13, 861-869

Macintosh RR, Mushin WW (1947): Observations on the epidural space. Anaesthesia 2 , 100-104

Marmarou A, Beaumont A: Physiology of the Cerebrospinal Fluid and Intracranial Pressure. In: Winn HR (Hrsg.): Youmans Neurological Surgery. Band 1, 5. Auflage; Saunders, Philadelphia 2004, 175-193

Misra A, Ganesh S, Shahiwala A, Shah SP (2003): Drug delivery to the central nervous system: a review. J Pharm Pharm Sci 6, 252-273

Miyan JA, Nabiyouni M, Zendah M (2003): Development of the brain: a vital role for cerebrospinal fluid. Can J Physiol Pharmacol 81, 317-328

Nedergaard M (2013): Garbage Truck of the Brain. Science 340, 1529-1530

Nilsson C, Ståhlberg F, Thomsen C, Henriksen O, Herning M, Owman C (1992): Circadian variation in human cerebrospinal fluid production measured by magnetic resonance imaging. Am J Physiol 262, R20-4 
Nitz WR, Bradley WG, Watanabe AS, Lee RR, Burgoyne B, O'Sullivan RM, Herbst MD (1992): Flow dynamics of cerebrospinal fluid: assessment with phase-contrast velocity MR imaging performed with retrospective cardiac gating. Radiology $\underline{183}$, 395405

O'Connell JEA (1943): The vascular factor in intracranial pressure and the maintenance of the cerebrospinal fluid circulation. Brain $66,204-228$

Reitan H (1941): On movements of fluid inside the cerebro-spinal space. Acta Radiol 22 , 762-779

Schaetz S, Uecker M: A Multi-GPU Programming Library for Real-Time Applications. In: Xiang Y, Stojmenovic I, Apduhan BO, Wang G, Nakano K, Zomaya A (Hrsg.): Algorithms and Architectures for Parallel Processing, ICA3PP 2012. Lecture Notes in Computer Science, vol. 7439. Springer, Berlin Heidelberg 2012, 114-128

Schroth G, Klose U (1992): Cerebrospinal fluid flow. I. Physiology of cardiac-related pulsation. Neuroradiology $\underline{35}, 1-9$

Shah JL (1981): Influence of cerebrospinal fluid on epidural pressure. Anaesthesia 36 , $627-631$

Silverberg GD, Mayo M, Saul T, Rubenstein E, McGuire D (2003): Alzheimer's disease, normal-pressure hydrocephalus, and senescent changes in CSF circulatory physiology: a hypothesis. Lancet Neurol $\underline{2}, 506-511$

Silverberg G, Mayo M, Saul T, Fellmann J, McGuire D (2006): Elevated cerebrospinal fluid pressure in patients with Alzheimer's disease. Cerebrospinal Fluid Res $\underline{3}, 7$

Spector R, Robert Snodgrass S, Johanson CE (2015): A balanced view of the cerebrospinal fluid composition and functions: Focus on adult humans. Exp Neurol 273, $57-68$

Spijkerman JM, Geurts LJ, Siero JCW, Hendrikse J, Luijten PR, Zwanenburg JJM (2018): Phase contrast MRI measurements of net cerebrospinal fluid flow through the cerebral aqueduct are confounded by respiration. J Magn Reson Imaging 49, 433-444

Stadlbauer A, Salomonowitz E, van der Riet W, Buchfelder M, Ganslandt O (2010): Insight into the patterns of cerebrospinal fluid flow in the human ventricular system using MR velocity mapping. Neuroimage 51, 42-52 
Stearns L, Boortz-Marx R, Du Pen S, Friehs G, Gordon M, Halyard M, Herbst L, Kiser J (2005): Intrathecal drug delivery for the management of cancer pain: a multidisciplinary consensus of best clinical practices. J Support Oncol $\underline{3}, 399-408$

Stringer MD, Restieaux M, Fisher AL, Crosado B (2012): The vertebral venous plexuses: the internal veins are muscular and external veins have valves. Clin Anat $\underline{25}, 609$ 618

Takizawa K, Matsumae M, Sunohara S, Yatsushiro S, Kuroda K (2017): Characterization of cardiac- and respiratory-driven cerebrospinal fluid motion based on asynchronous phase-contrast magnetic resonance imaging in volunteers. Fluids Barriers CNS $\underline{14}, 25$

Tan Z, Roeloffs V, Voit D, Joseph AA, Untenberger M, Merboldt KD, Frahm J (2017): Model-based reconstruction for real-time phase-contrast flow MRI: Improved spatiotemporal accuracy. Magn Reson Med 77, 1082-1093

Trepel M: Neuroanatomie: Struktur und Funktion. 6. Auflage; Elsevier, Urban \& Fischer, München 2015

Tumani H, Huss A, Bachhuber F (2017): The cerebrospinal fluid and barriers - anatomic and physiologic considerations. Handb Clin Neurol 146, 21-32

Uecker M, Zhang S, Voit D, Karaus A, Merboldt K-D, Frahm J (2010): Real-time MRI at a resolution of $20 \mathrm{~ms}$. NMR Biomed $\underline{23}, 986-994$

Untenberger M, Tan Z, Voit D, Joseph AA, Roeloffs V, Merboldt KD, Schätz S, Frahm $\mathrm{J}$ (2016): Advances in real-time phase-contrast flow MRI using asymmetric radial gradient echoes. Magn Reson Med 75, 1901-1908

Usubiaga JE, Moya F, Usubiaga LE (1967): Effect of thoracic and abdominal pressure changes on the epidural space pressure. Br J Anaesth 39, 612-618

Weed LH (1929): Some limitations of the Monro-Kellie hypothesis. Arch Surg 18, 10491068

Williams B (1976): Cerebrospinal fluid pressure changes in response to coughing. Brain 99, 331-346 
Williams B (1981 a): Simultaneous cerebral and spinal fluid pressure recordings. 2. Cerebrospinal dissociation with lesions at the foramen magnum. Acta Neurochir (Wien) $\underline{59}, 123-142$

Williams B (1981 b): Simultaneous cerebral and spinal fluid pressure recordings. I. Technique, physiology, and normal results. Acta Neurochir (Wien) $\underline{58}, 167-185$

Yamada S, Miyazaki M, Yamashita Y, Ouyang C, Yui M, Nakahashi M, Shimizu S, Aoki I, Morohoshi Y, McComb JG (2013): Influence of respiration on cerebrospinal fluid movement using magnetic resonance spin labeling. Fluids Barriers CNS 19, 36

Yildiz S, Thyagaraj S, Jin N, Zhong X, Heidari Pahlavian S, Martin BA, Loth F, Oshinski J, Sabra KG (2017): Quantifying the influence of respiration and cardiac pulsations on cerebrospinal fluid dynamics using real-time phase-contrast MRI. J Magn Reson Imaging 46, 431-439 


\section{Danksagung}

Frau PD Dr. Dreha-Kulaczewski danke ich für die Überlassung des Themas und die Betreuung sowie für die freundliche Unterstützung bei der Erstellung meiner Arbeit.

Herrn Prof. Dr. Jens Frahm möchte ich für die Ermöglichung und Begleitung meiner Arbeit in seiner Arbeitsgruppe (Biomedizinische NMR) danken. Mit freundlicher und konstruktiver Kritik war er ein wertvoller Ansprechpartner.

Herrn Dr. Klaus-Dietmar Merboldt und Herrn Dr. Arun Antony Joseph danke ich für die Durchführungen der Messungen und die freundliche und hilfsbereite Unterstützung in physikalischen Fragen.

Frau Dr. Sabine Hofer, Herrn Jakob Klosowski, Herrn Jost Kollmeier und nochmals Herrn Dr. Klaus-Dietmar Merboldt gebührt mein besonderer Dank für die vielen hilfreichen Hinweise und die wertvolle, konstruktive und geduldige Unterstützung bei der Durchsicht meiner Arbeit.

Frau Dr. Renate Schweizer danke ich für die in Diskussionen geäußerten Anregungen und Hinweise.

Herrn Kurt Böhm danke ich für die unerlässliche IT-Betreuung.

Mein Dank gilt auch allen Probanden, die sich für die Studie zur Verfügung gestellt haben. 